\title{
Synthesis of Sugar Based Enones and their Transformation into 3,5-Disubstituted Furans and 2-Acyl-Substituted 1,2,3-Trideoxy Sugars in Presence of Lewis Acids
}

\author{
Nazar Hussain, ${ }^{\dagger} \pitchfork$ Monika Bhardwaj,,$\dagger$ Ajaz Ahmed, $\dagger, \star$ and Debaraj Mukherjee ${ }^{\dagger, \uparrow *}$ \\ $\dagger$ Natural Product Chemistry Division, Indian Institute of Integrative Medicine (IIIM), \\ Jammu, India. \\ \$Academy of Scientific and Innovative Research (AcSIR-IIIM), Jammu-180001, India.
}

Page No.

1. General Consideration SI-2

2. Experimental procedures for all products SI-2

3. Optimization table for compound 6a-6c SI-3

$\begin{array}{ll}\text { 4. Characterization data: products } & \text { SI-7 }\end{array}$

$\begin{array}{ll}\text { 5. NMR spectra } & \text { SI-27 }\end{array}$

$\begin{array}{ll}\text { 6. HSQC/NOESY of compound 6a, } 8 & \text { SI-64,74 }\end{array}$ 


\section{General Consideration}

${ }^{1} \mathrm{H}$ and ${ }^{13} \mathrm{C}$ NMR spectra were recorded using 400 and $500 \mathrm{MHz}$ spectrometers with TMS as internal standard. Chemical shifts are expressed in parts per million ( $\delta \mathrm{ppm})$. Silica gel coated aluminum plates were used for TLC. The products were purified by column chromatography on silica gel (60-120 mesh) using petroleum ether-ethyl acetate as the eluent to obtain the pure products. Exact masses of all products were derived by using HRMS having QTOF analyzer. Reagents used were mostly purchased from Sigma Aldrich.

Note: Moisture free reagent grade (95-97 \%) formic acids needs to be used for the synthesis of enones.

\section{Experimental procedures for all products}

\subsection{General procedure for sugar based Arylones (3a-3k).}

In an oven dried single neck round bottom flask charged with magnetic bead and flashed three times with $\mathrm{N}_{2}, 2$-iodo-glycal (1 equiv), $\mathrm{Pd}(\mathrm{OAc})_{2}$ (5 mol\%, 0.05 equiv), $\mathrm{PPh}_{3}$ ( 10 mol\% $\% 0.1$ equiv) in $5 \mathrm{~mL}$ of dry toluene were added. In the same solution boronic acids (1.2 equiv), DCC ( 2 equiv) and $\mathrm{K}_{2} \mathrm{CO}_{3}$ (2 equiv) were also added and the reaction mixture was sealed with rubber septum. Formic acid ( 2 equiv), triethyl amine ( 2 equiv) were added via syringe sequentially and stirred the reaction mixture at $\mathrm{rt}$ under $\mathrm{N}_{2}$ atmosphere until complete consumption of starting material was observed by TLC analysis. Then the reaction mixture was diluted with chilled $10 \mathrm{~mL}$ of toluene/hexane $(1: 1)$ to precipitate the DCU formed from DCC. This precipitation process was repeated at least three times before filtration through a filter paper. The filtrate was washed with $20 \mathrm{~mL}$ of ethyl acetate and brine solution. The organic layer was dried over sodium sulphate and evaporated in vacuo. The residue left was purified by column chromatography over silica gel (60-120 mesh) using pet ether/ ethyl acetate as eluent.

\subsection{General procedure for sugar based ynones (5a-5f).}


In an oven dried single neck round bottom flask charged with magnetic bead and flashed three times with $\mathrm{N}_{2}$, 2-iodo-glycal (1 equiv), $\mathrm{Pd}(\mathrm{OAc})_{2}$ (5 mol\%, 0.05 equiv), $\mathrm{PPh}_{3}(10$ mol\% , 0.1 equiv) in $5 \mathrm{~mL}$ of dry toluene were added. In the same solution acetylene (1.2 equiv), and DCC (2 equiv) were also added and the reaction mixture was sealed with rubber septa. Formic acid (2 equiv), triethyl amine (2 equiv) were added via syringe sequentially and stirred the reaction mixture at $\mathrm{rt}$ under $\mathrm{N}_{2}$ atmosphere until complete consumption of starting material was observed by TLC analysis. Then the reaction mixture was diluted with chilled $10 \mathrm{~mL}$ of toluene/hexane (1:1) to precipitate the DCU formed from DCC. This precipitation process was repeated at least three times before filtration through a filter paper. The filtrate was washed with $20 \mathrm{~mL}$ of ethyl acetate and brine solution. The organic layer was dried over sodium sulphate and evaporated in vacuo. The residue left was purified by column chromatography over silica gel (60-120 mesh) using pet ether/ ethyl acetate as eluent.

\section{Table 1 Optimization of reaction condition}

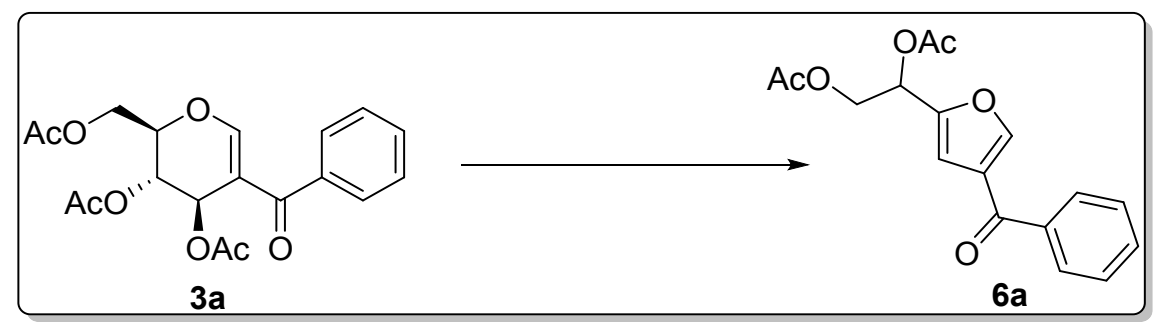

\begin{tabular}{|c|c|c|c|c|}
\hline Entry & Lewis acid (mol \%) & Time (h) & Temperature & Yield \% \\
\hline 1 & $\mathrm{BF}_{3}(\mathrm{OEt})_{2}(100)$ & 6 & $30^{\circ} \mathrm{C}$ & 43 \\
\hline 2 & $\mathrm{BF}_{3}(\mathrm{OEt})_{2}(100)$ & 6 & $50^{\circ} \mathrm{C}$ & 20 \\
\hline 3 & $\mathrm{BF}_{3}(\mathrm{OEt})_{2}(50)$ & 6 & $50^{\circ} \mathrm{C}$ & 23 \\
\hline 4 & TfOH (10) & 1 & $30^{\circ} \mathrm{C}$ & 53 \\
\hline 5 & TfOH (10) & 1 & $30^{\circ} \mathrm{C}$ & 53 \\
\hline 4 & TfOH (10) & 1 & $20^{\circ} \mathrm{C}$ & 57 \\
\hline 5 & TfOH (20) & $20 \mathrm{~min}$ & $20^{\circ} \mathrm{C}$ & 63 \\
\hline
\end{tabular}




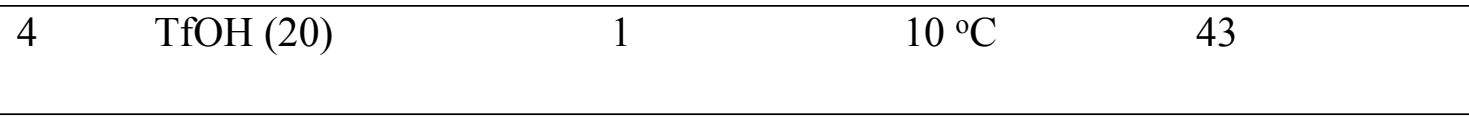

\subsection{Procedure for the synthesis 6a-6c}

In an oven dried single neck round bottom flask charged with magnetic bead and flashed three times with $\mathrm{N}_{2}, \mathbf{3 a}, \mathbf{3 j}$, 3k (1 equiv) in $5 \mathrm{~mL}$ dichloromethane at $0{ }^{\circ} \mathrm{C}$ and $\mathrm{TfOH}(0.2$ equiv) were added at $0{ }^{\circ} \mathrm{C}$. Remove the reaction from ice bath after 5 minutes and stirred the reaction mixture at $\mathrm{rt}$ until complete consumption of starting material was observed by TLC analysis. Then the reaction mixture was diluted with $5 \mathrm{~mL}$ of DCM and washed the reaction mixture with saturated sodium bicarbonate solution $(5 \mathrm{~mL})$. The organic layer was dried over sodium sulphate and evaporated in vacuo. The residue left was purified by column chromatography over silica gel (60-120 mesh) using pet ether/ethyl acetate (10:1) as eluent to obtain the compound $\mathbf{6 a - 6} \mathbf{c}$ as colorless oil.

\subsection{Procedure for the synthesis 7}

In an oven dried single neck round bottom flask charged with magnetic bead and flashed three times with $\mathrm{N}_{2}, \mathbf{3 h}(0.09 \mathrm{mmol})$ in $5 \mathrm{~mL}$ dichloromethane at $0{ }^{\circ} \mathrm{C}$ and $\mathrm{BF}_{3}(\mathrm{OEt})_{2}(0.09$ mmol) were added. Remove the reaction from ice bath after 5 minutes and stirred the reaction mixture at rt until complete consumption of starting material was observed by TLC analysis. Then the reaction mixture was diluted with $5 \mathrm{~mL}$ of DCM and washed the reaction mixture with saturated sodium bicarbonate solution $(10 \mathrm{~mL})$. The organic layer was dried over sodium sulphate and evaporated in vacuo. The residue left was purified by column chromatography over silica gel (60-120 mesh) using pet ether/ethyl acetate (85:15) as eluent to obtain the compound 7 as colorless oil (18 mg, 58\%).

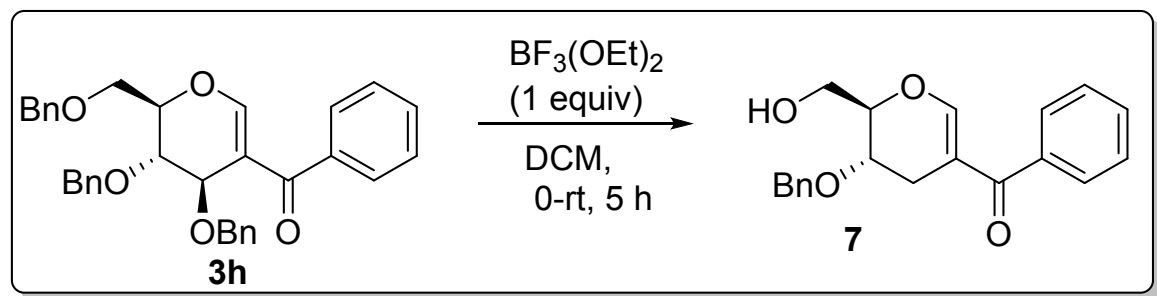

\subsection{Procedure for the synthesis $8,9,10,10 a \& 11$.}

- Compound 8 was synthesized using $3 \mathbf{a}(0.13 \mathrm{mmol})$, in $5 \mathrm{~mL}$ of dichloromethane, $\mathrm{AlCl}_{3}(0.19 \mathrm{mmol})$ was added. Stirred the reaction mixture at $\mathrm{rt}$ until complete consumption of starting material was observed by TLC analysis. Then the reaction 
mixture was diluted with $5 \mathrm{~mL}$ of DCM and washed the reaction mixture with saturated sodium bicarbonate solution $(10 \mathrm{~mL})$. The organic layer was dried over sodium sulphate and evaporated in vacuo. The residue left was purified by column chromatography over silica gel (60-120 mesh) using pet ether/ethyl acetate (90:10) as eluent to obtain the compound 8 as colorless oil ( $30 \mathrm{mg}, 64 \%$ ).

- Compound 9 was synthesized using 3a $(0.13 \mathrm{mmol})$, in $5 \mathrm{~mL}$ of dichloromethane, 2-bromo thiophenol $(0.15 \mathrm{mmol})$ and $\mathrm{BF}_{3}(\mathrm{OEt})_{2}(0.13 \mathrm{mmol})$ were added. Stirred the reaction mixture at $\mathrm{rt}$ until complete consumption of starting material was observed by TLC analysis. Then the reaction mixture was diluted with $5 \mathrm{~mL}$ of DCM and washed the reaction mixture with saturated sodium bicarbonate solution $(10 \mathrm{~mL})$. The organic layer was dried over sodium sulphate and evaporated in vacuo. The residue left was purified by column chromatography over silica gel (60120 mesh) using pet ether/ethyl acetate (90:10) as eluent to obtain the compound 9 as colorless oil (52 $\mathrm{mg}, 78 \%$ ).

- Compound 10 and 10a was synthesized using $3 \mathbf{f}$ and 3k (1 equiv), in $5 \mathrm{~mL}$ of dichloromethane, allyl trimethylsilane (1.2 equiv) and $\mathrm{BF}_{3}(\mathrm{OEt})_{2}$ ( 1 equiv) were added. Stirred the reaction mixture at rt until complete consumption of starting material was observed by TLC analysis. Then the reaction mixture was diluted with $5 \mathrm{~mL}$ of DCM and washed the reaction mixture with saturated sodium bicarbonate solution $(10 \mathrm{~mL})$. The organic layer was dried over sodium sulphate and evaporated in vacuo. The residue left was purified by column chromatography over silica gel (60-120 mesh) using pet ether/ethyl acetate (90:10) as eluent to obtain the compound 10 and 10a as colorless oil.

- Compound 11 was synthesized using $3 \mathbf{3 a}(0.09 \mathrm{mmol})$, in $5 \mathrm{~mL}$ of dichloromethane, triethyl silylhydride $(0.11 \mathrm{mmol})$ and $\mathrm{BF}_{3}(\mathrm{OEt})_{2}(0.11 \mathrm{mmol})$ were added. Stirred the reaction mixture at $\mathrm{rt}$ until complete consumption of starting material was observed by TLC analysis. Then the reaction mixture was diluted with $5 \mathrm{~mL}$ of DCM and washed the reaction mixture with saturated sodium bicarbonate solution $(10 \mathrm{~mL})$. The organic layer was dried over sodium sulphate and evaporated in vacuo. The residue left was purified by column chromatography over silica gel (60- 
120 mesh) using pet ether/ethyl acetate (90:10) as eluent to obtain the compound 11 as colorless oil (26 mg, 66\%).

\subsection{Procedure for the synthesis of compound $12 \mathrm{a} / \mathrm{b}$}

- Compound $\mathbf{1 2 a} / \mathbf{b}$ was synthesized using $\mathbf{3 h}(0.09 \mathrm{mmol})$, in $5 \mathrm{~mL}$ of ethanol, sodium borohydride $(0.57 \mathrm{mmol})$ and $\mathrm{CeCl}_{3}(0.23 \mathrm{mmol})$ were added slowly at room temperature. Stirred the reaction mixture at $\mathrm{rt}$ until complete consumption of starting material was observed by TLC analysis. Then the reaction mixture was diluted with $5 \mathrm{~mL}$ of ethyl acetate $(20 \mathrm{~mL})$ and washed the reaction mixture with saturated sodium bicarbonate solution $(10 \mathrm{~mL})$. The organic layer was dried over sodium sulphate and evaporated in vacuo. The residue left was purified by column chromatography over silica gel (60-120 mesh) using pet ether/ethyl acetate (90:10) as eluent to obtain the compound $\mathbf{1 2 a} \mathbf{2} \mathbf{1 2 b}$ as gummy liquid (78 $\mathrm{mg}, 77 \%$ ).

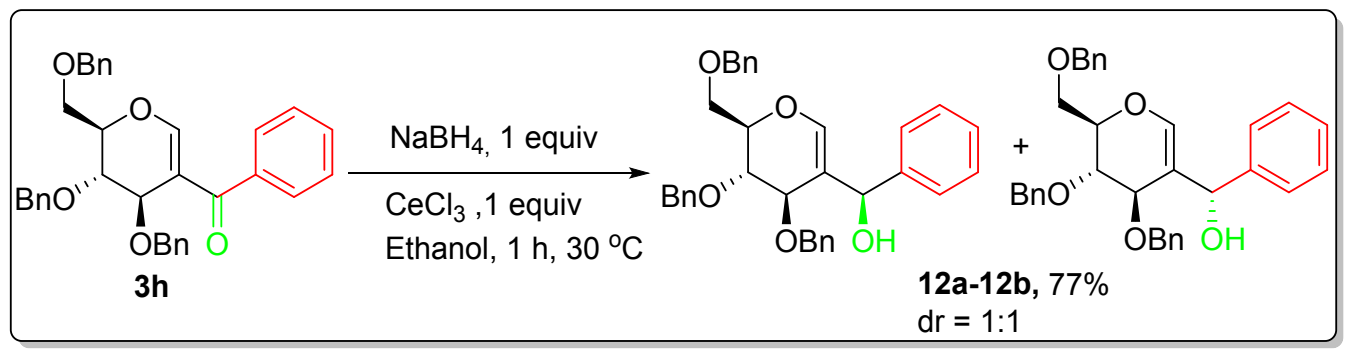

\subsection{Typical Procedure for the synthesis of 3a (1 mmol scale)}

- In an oven dried single neck round bottom flask charged with magnetic bead and flashed three times with $\mathrm{N}_{2}$, 2-iodo-glycal (1 mmol), $\mathrm{Pd}(\mathrm{OAc})_{2}(0.05 \mathrm{mmol}), \mathrm{PPh}_{3}$ $(0.1 \mathrm{mmol})$ in $7 \mathrm{~mL}$ of dry toluene were added. In the same solution boronic acids (1.2 mmol), DCC (2 mmol) and $\mathrm{K}_{2} \mathrm{CO}_{3}(2 \mathrm{mmol})$ were also added and the reaction mixture was sealed with rubber septum. Formic acid ( $2 \mathrm{mmol})$, triethyl amine (2 mmol) were added via syringe sequentially and stirred the reaction mixture at $\mathrm{rt}$ under $\mathrm{N}_{2}$ atmosphere until complete consumption of starting material was observed by TLC analysis. Then the reaction mixture was diluted with chilled $10 \mathrm{~mL}$ of toluene/hexane $(1: 1)$ to precipitate the DCU formed from DCC. This precipitation process was repeated at least three times before filtration through a filter paper. The filtrate was washed with $20 \mathrm{~mL}$ of ethyl acetate and brine solution. The organic layer was dried over sodium sulphate and evaporated in vacuo. The residue left was 
purified by column chromatography over silica gel (60-120 mesh) using pet ether/ ethyl acetate as eluent to obtained 3a as yellow oil (260 mg, 69\%)

\section{Characterization data: products}

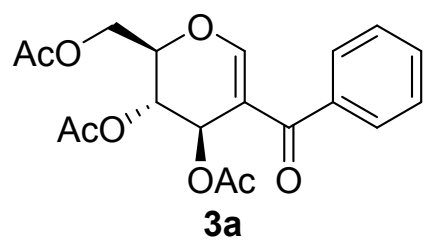

2-(acetoxymethyl)-5-benzoyl-3,4-dihydro-2H-pyran-3,4-diyl diacetate (3a). Prepared according to general procedure 2.1. In an oven dried single neck round bottom flask charged with magnetic bead and flashed three times with $\mathrm{N}_{2}, 2$-iodo-tri- $O$-acetyl-D-glucal $(0.25 \mathrm{mmol})$, $\mathrm{Pd}(\mathrm{OAc})_{2}\left(0.012 \mathrm{mmol}, 0.05\right.$ equiv), $\mathrm{PPh}_{3}(0.025 \mathrm{mmol}, 0.1$ equiv) in $5 \mathrm{~mL}$ of dry toluene were added. In the same solution phenyl boronic acid ( $0.3 \mathrm{mmol}, 1.2$ equiv), DCC ( $0.5 \mathrm{mmol}, 2$ equiv) and $\mathrm{K}_{2} \mathrm{CO}_{3}(0.5 \mathrm{mmol}, 2$ equiv) were also added and the reaction mixture was sealed with rubber septa. Formic acid ( $0.5 \mathrm{mmol}, 2$ equiv), triethyl amine ( $0.5 \mathrm{mmol}, 2$ equiv) were added via syringe sequentially and stirred the reaction mixture at $\mathrm{rt}$ under $\mathrm{N}_{2}$ atmosphere until complete consumption of starting material was observed by TLC analysis. Then the reaction mixture was diluted with chilled $10 \mathrm{~mL}$ of toluene/hexane (1:1) to precipitate the DCU formed from DCC. This precipitation process was repeated at least three times before filtration through a filter paper. The filtrate was washed with $20 \mathrm{~mL}$ of ethyl acetate and brine solution. The residue left was purified by column chromatography over silica gel (60-120 mesh) using pet ether/ ethyl acetate (82:1.6) as eluent as yellow oil ( $72 \%, 69 \mathrm{mg}$ ). ${ }^{1} \mathrm{H}$ NMR (400 MHz, $\left.\mathrm{CDCl}_{3}\right) \delta 7.66(\mathrm{~d}, J=7.5 \mathrm{~Hz}, 2 \mathrm{H}), 7.53$ (t, $J=7.2$ $\mathrm{Hz}, 1 \mathrm{H}), 7.45(\mathrm{t}, J=7.4 \mathrm{~Hz}, 2 \mathrm{H}), 7.40(\mathrm{~s}, 1 \mathrm{H}), 5.98(\mathrm{~d}, J=2.9 \mathrm{~Hz}, 1 \mathrm{H}), 5.30(\mathrm{~d}, J=3.3 \mathrm{~Hz}, 1 \mathrm{H})$, $4.66-4.56(\mathrm{~m}, 2 \mathrm{H}), 4.18(\mathrm{~d}, J=8.8 \mathrm{~Hz}, 1 \mathrm{H}), 2.12$ (s, 3H), 2.09 (s, 3H), $2.03(\mathrm{~s}, 3 \mathrm{H}) .{ }^{13} \mathrm{C}$ NMR $\left(101 \mathrm{MHz}, \mathrm{CDCl}_{3}\right) \delta 192.9,170.4,169.6,169.4,159.2,138.2,131.9,128.9,128.5,113.5,75.1$, 66.2, 62.8, 60.8, 20.7, 20.7, 20.6. IR $\left(\mathrm{CHCl}_{3} \mathrm{~cm}^{-1}\right): v$ 1745, 1621; HRMS (ESI+): $\mathrm{m} / \mathrm{z}$ calcd. For $\mathrm{C}_{19} \mathrm{H}_{20} \mathrm{NaO}_{8}(\mathrm{M}+\mathrm{Na})^{+}$399.1056, found 399.1046. $[\alpha]_{\mathrm{D}}+35.4$ (c 1, $\left.\mathrm{CHCl}_{3}\right)$. 


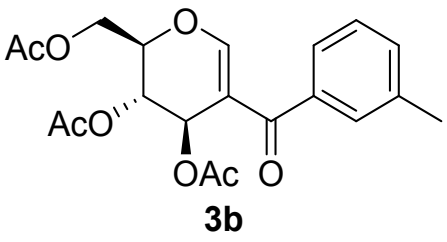

2-(acetoxymethyl)-5-(3-methylbenzoyl)-3,4-dihydro-2H-pyran-3,4-diyl diacetate (3b). Prepared according to general procedure 2.1. In an oven dried single neck round bottom flask charged with magnetic bead and flashed three times with $\mathrm{N}_{2}, 2$-iodo-tri-O-acetyl-D-glucal $(0.25$ mmol), $\mathrm{Pd}(\mathrm{OAc})_{2}\left(0.012 \mathrm{mmol}, 0.05\right.$ equiv), $\mathrm{PPh}_{3}(0.025 \mathrm{mmol}, 0.1$ equiv $)$ in $5 \mathrm{~mL}$ of dry toluene were added. In the same solution 3-methyl phenyl boronic acid ( $0.3 \mathrm{mmol}, 1.2$ equiv), DCC ( 0.5 mmol, 2 equiv) and $\mathrm{K}_{2} \mathrm{CO}_{3}(0.5 \mathrm{mmol}, 2$ equiv) were also added and the reaction mixture was sealed with rubber septa. Formic acid ( $0.5 \mathrm{mmol}, 2$ equiv), triethyl amine ( $0.5 \mathrm{mmol}, 2$ equiv) were added via syringe sequentially and stirred the reaction mixture at $\mathrm{rt}$ under $\mathrm{N}_{2}$ atmosphere until complete consumption of starting material was observed by TLC analysis. Then the reaction mixture was diluted with chilled $10 \mathrm{~mL}$ of toluene/hexane (1:1) to precipitate the DCU formed from DCC. This precipitation process was repeated at least three times before filtration through a filter paper. The filtrate was washed with $20 \mathrm{~mL}$ of ethyl acetate and brine solution. The organic layer was dried over sodium sulphate and evaporated in vacuo. The residue left was purified by column chromatography over silica gel (60-120 mesh) using pet ether/ ethyl acetate (8:1.6) as eluent as yellow oil ( 70\%, $69 \mathrm{mg}){ }^{1} \mathrm{H} \mathrm{NMR}\left(400 \mathrm{MHz}, \mathrm{CDCl}_{3}\right) \delta 7.38(\mathrm{dd}, J=7.7,4.0 \mathrm{~Hz}, 2 \mathrm{H})$, $7.33(\mathrm{~s}, 1 \mathrm{H}), 7.26(\mathrm{~d}, J=6.1 \mathrm{~Hz}, 2 \mathrm{H}), 5.91(\mathrm{~d}, J=3.7 \mathrm{~Hz}, 1 \mathrm{H}), 5.22$ (t, $J=3.9 \mathrm{~Hz}, 1 \mathrm{H}), 4.60-$ $4.46(\mathrm{~m}, 2 \mathrm{H}), 4.11$ (dd, $J=11.4,3.1 \mathrm{~Hz}, 1 \mathrm{H}), 2.34$ (s, 3H), 2.05 (s, 3H), $2.02(\mathrm{~s}, 3 \mathrm{H}), 1.96(\mathrm{~s}, 3 \mathrm{H})$. ${ }^{13} \mathrm{C}$ NMR $\left(101 \mathrm{MHz}, \mathrm{CDCl}_{3}\right) \delta 193.1,170.4,169.6,169.4,159.1,138.4,138.2,132.6,129.3$, 128.3, 126.0, 113.6, 75.1, 66.2, 62.9, 60.8, 21.3, 20.8, 20.7, 20.7. IR $\left(\mathrm{CHCl}_{3} \mathrm{~cm}^{-1}\right): v 1742,1626$; HRMS (ESI+): $\mathrm{m} / \mathrm{z}$ calcd. For $\mathrm{C}_{20} \mathrm{H}_{22} \mathrm{NaO}_{8}(\mathrm{M}+\mathrm{Na})^{+} 413.1212$, found 413.1220. $[\alpha]_{\mathrm{D}}+60.4(\mathrm{c} 1$, $\left.\mathrm{CHCl}_{3}\right)$.

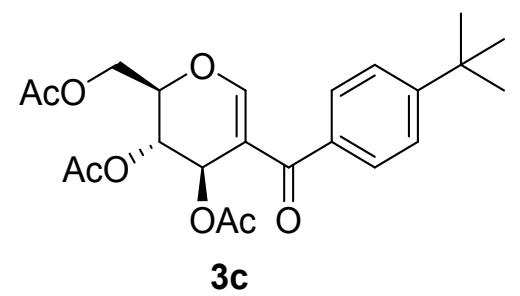


2-(acetoxymethyl)-5-(4-(tert-butyl) benzoyl)-3,4-dihydro-2H-pyran-3,4-diyl diacetate (3c).

Prepared according to general procedure 2.1. In an oven dried single neck round bottom flask charged with magnetic bead and flashed three times with $\mathrm{N}_{2}, 2$-iodo-tri- $O$-acetyl-D-glucal $(0.25$ mmol), $\mathrm{Pd}(\mathrm{OAc})_{2}\left(0.012 \mathrm{mmol}, 0.05\right.$ equiv), $\mathrm{PPh}_{3}(0.025 \mathrm{mmol}, 0.1$ equiv $)$ in $5 \mathrm{~mL}$ of dry toluene were added. In the same solution 4-tert-butyl phenyl boronic acid ( $0.3 \mathrm{mmol}, 1.2$ equiv), DCC ( 0.5 mmol, 2 equiv) and $\mathrm{K}_{2} \mathrm{CO}_{3}$ (0.5 mmol, 2 equiv) were also added and the reaction mixture was sealed with rubber septa. Formic acid ( $0.5 \mathrm{mmol}, 2$ equiv), triethyl amine $(0.5 \mathrm{mmol}, 2$ equiv) were added via syringe sequentially and stirred the reaction mixture at $\mathrm{rt}$ under $\mathrm{N}_{2}$ atmosphere until complete consumption of starting material was observed by TLC analysis. Then the reaction mixture was diluted with chilled $10 \mathrm{~mL}$ of toluene/hexane (1:1) to precipitate the DCU formed from DCC. This precipitation process was repeated at least three times before filtration through a filter paper. The filtrate was washed with $20 \mathrm{~mL}$ of ethyl acetate and brine solution. The organic layer was dried over sodium sulphate and evaporated in vacuo. The residue left was purified by column chromatography over silica gel (60-120 mesh) using pet ether/ ethyl acetate (8:1.5) as eluent as yellow oil $(68 \%, 74 \mathrm{mg}) .{ }^{1} \mathrm{H}$ NMR $\left(400 \mathrm{MHz}, \mathrm{CDCl}_{3}\right) \delta 7.64(\mathrm{~d}, J=8.4 \mathrm{~Hz}, 2 \mathrm{H}), 7.47$ $(\mathrm{dd}, J=9.4,5.1 \mathrm{~Hz}, 2 \mathrm{H}), 7.43(\mathrm{~s}, 1 \mathrm{H}), 6.00(\mathrm{~d}, J=2.8 \mathrm{~Hz}, 1 \mathrm{H}), 5.31(\mathrm{t}, J=3.9 \mathrm{~Hz}, 1 \mathrm{H}), 4.61(\mathrm{~m}$, 2H), $4.19(\mathrm{dd}, J=11.9,3.7 \mathrm{~Hz}, 1 \mathrm{H}), 2.14(\mathrm{~s}, 3 \mathrm{H}), 2.10(\mathrm{~s}, 3 \mathrm{H}), 2.03(\mathrm{~s}, 3 \mathrm{H}), 1.36(\mathrm{~s}, 9 \mathrm{H}){ }^{13} \mathrm{C} \mathrm{NMR}$ $\left(101 \mathrm{MHz}, \mathrm{CDCl}_{3}\right) \delta 192.8,170.5,169.7,169.5,158.8,155.7,135.6,135.3,133.7,129.0,125.4$, 125.0, 125.0, 113.4, 75.0, 66.3, 62.9, 60.8, 35.0, 31.2, 31.2, 20.8, 20.7. IR $\left(\mathrm{CHCl}_{3} \mathrm{~cm}^{-1}\right): v 1749$, 1623; HRMS (ESI+): $\mathrm{m} / \mathrm{z}$ calcd. For $\mathrm{C}_{23} \mathrm{H}_{28} \mathrm{NaO}_{8}(\mathrm{M}+\mathrm{Na})^{+} 455.1682$, found 455.1678. $[\alpha]_{\mathrm{D}}+30.6$ (c $1, \mathrm{CHCl}_{3}$ ).

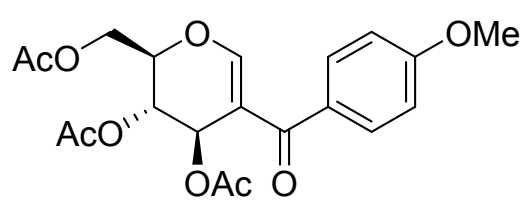

3d

\section{2-(acetoxymethyl)-5-(4-methoxybenzoyl)-3,4-dihydro-2H-pyran-3,4-diyl diacetate (3d).}

Prepared according to general procedure 2.1. In an oven dried single neck round bottom flask charged with magnetic bead and flashed three times with $\mathrm{N}_{2}, 2$-iodo-tri- $O$-acetyl-D-glucal $(0.25$ $\mathrm{mmol}), \mathrm{Pd}(\mathrm{OAc})_{2}\left(0.012 \mathrm{mmol}, 0.05\right.$ equiv), $\mathrm{PPh}_{3}(0.025 \mathrm{mmol}, 0.1$ equiv $)$ in $5 \mathrm{~mL}$ of dry toluene were added. In the same solution 4-methoxy phenyl boronic acid ( $0.3 \mathrm{mmol}, 1.2$ equiv), DCC ( 0.5 mmol, 2 equiv) and $\mathrm{K}_{2} \mathrm{CO}_{3}(0.5 \mathrm{mmol}, 2$ equiv) were also added and the reaction mixture was 
sealed with rubber septa. Formic acid ( $0.5 \mathrm{mmol}, 2$ equiv), triethyl amine ( $0.5 \mathrm{mmol}, 2$ equiv) were added via syringe sequentially and stirred the reaction mixture at $\mathrm{rt}$ under $\mathrm{N}_{2}$ atmosphere until complete consumption of starting material was observed by TLC analysis. Then the reaction mixture was diluted with chilled $10 \mathrm{~mL}$ of toluene/hexane (1:1) to precipitate the DCU formed from DCC. This precipitation process was repeated at least three times before filtration through a filter paper. The filtrate was washed with $20 \mathrm{~mL}$ of ethyl acetate and brine solution. The organic layer was dried over sodium sulphate and evaporated in vacuo. The residue left was purified by column chromatography over silica gel (60-120 mesh) using pet ether/ ethyl acetate (8:1.5) as eluent as yellow oil $(67 \%, 69 \mathrm{mg}) .{ }^{1} \mathrm{H}$ NMR $\left(400 \mathrm{MHz}, \mathrm{CDCl}_{3}\right) \delta 7.43(\mathrm{~s}, 1 \mathrm{H}), 7.38-7.31(\mathrm{~m}$, 2H), $7.21(\mathrm{~m}, 2 \mathrm{H}), 7.06(\mathrm{~m}, 1 \mathrm{H}), 6.01-5.92(\mathrm{~m}, 1 \mathrm{H}), 5.28(\mathrm{t}, J=3.7 \mathrm{~Hz}, 1 \mathrm{H}), 4.65-4.56(\mathrm{~m}$, 2H), $4.17(\mathrm{dd}, J=11.6,3.1 \mathrm{~Hz}, 1 \mathrm{H}), 3.85(\mathrm{~s}, 3 \mathrm{H}), 2.12(\mathrm{~s}, 3 \mathrm{H}), 2.09(\mathrm{~s}, 3 \mathrm{H}), 2.04(\mathrm{~s}, 3 \mathrm{H}) .{ }^{13} \mathrm{C}$ NMR $\left(101 \mathrm{MHz}, \mathrm{CDCl}_{3}\right) \delta 192.8,170.4,169.6,169.4,159.7,139.4,129.4,129.2,128.0,121.4,120.4$, 118.4, 118.1, 113.6, 113.4, 75.1, 66.2, 62.7, 60.8, 55.5, 24.9, 20.8, 20.7. IR $\left(\mathrm{CHCl}_{3} \mathrm{~cm}^{-1}\right): v 1747$, 1625; HRMS (ESI+): $\mathrm{m} / \mathrm{z}$ calcd. For $\mathrm{C}_{20} \mathrm{H}_{22} \mathrm{NaO}_{9}(\mathrm{M}+\mathrm{Na})^{+} 429.1162$, found 429.1168. $[\alpha]_{\mathrm{D}}+58.5$ (c $1, \mathrm{CHCl}_{3}$ ).

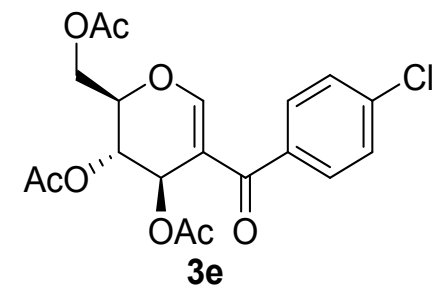

2-(acetoxymethyl)-5-(4-chlorobenzoyl)-3,4-dihydro-2H-pyran-3,4-diyl diacetate (3e). Prepared according to general procedure 2.1. In an oven dried single neck round bottom flask charged with magnetic bead and flashed three times with $\mathrm{N}_{2}$, 2-iodo-tri- $O$-acetyl-D-glucal $(0.25$ $\mathrm{mmol}), \mathrm{Pd}(\mathrm{OAc})_{2}\left(0.012 \mathrm{mmol}, 0.05\right.$ equiv), $\mathrm{PPh}_{3}(0.025 \mathrm{mmol}, 0.1$ equiv) in $5 \mathrm{~mL}$ of dry toluene were added. In the same solution 4-chloro- phenyl boronic acid ( $0.3 \mathrm{mmol}, 1.2$ equiv), DCC ( 0.5 mmol, 2 equiv) and $\mathrm{K}_{2} \mathrm{CO}_{3}(0.5 \mathrm{mmol}, 2$ equiv) were also added and the reaction mixture was sealed with rubber septa. Formic acid ( $0.5 \mathrm{mmol}, 2$ equiv), triethyl amine ( $0.5 \mathrm{mmol}, 2$ equiv) were added via syringe sequentially and stirred the reaction mixture at $\mathrm{rt}$ under $\mathrm{N}_{2}$ atmosphere until complete consumption of starting material was observed by TLC analysis. Then the reaction mixture was diluted with chilled $10 \mathrm{~mL}$ of toluene/hexane (1:1) to precipitate the DCU formed from DCC. This precipitation process was repeated at least three times before filtration through a 
filter paper. The filtrate was washed with $20 \mathrm{~mL}$ of ethyl acetate and brine solution. The organic layer was dried over sodium sulphate and evaporated in vacuo. The residue left was purified by column chromatography over silica gel (60-120 mesh) using pet ether/ ethyl acetate (8:1.5) as eluent as yellow oil ( $63 \%, 65 \mathrm{mg}) .{ }^{1} \mathrm{H}$ NMR $\left(400 \mathrm{MHz}, \mathrm{CDCl}_{3}\right) \delta 7.63(\mathrm{~d}, J=8.4 \mathrm{~Hz}, 2 \mathrm{H}), 7.44$ (d, $J=8.6 \mathrm{~Hz}, 2 \mathrm{H}), 7.38(\mathrm{~s}, 1 \mathrm{H}), 6.04-5.88(\mathrm{~m}, 1 \mathrm{H}), 5.28$ (t, $J=3.7 \mathrm{~Hz}, 1 \mathrm{H}), 4.63$ (ddd, $J=11.0$, 9.7, $5.8 \mathrm{~Hz}, 2 \mathrm{H}), 4.21-4.14(\mathrm{~m}, 1 \mathrm{H}), 2.13$ (s, 3H), 2.10 (s, 3H), 2.04 (s, 3H). ${ }^{13} \mathrm{C}$ NMR $(101 \mathrm{MHz}$, $\left.\mathrm{CDCl}_{3}\right) \delta 191.7,170.4,169.5,169.4,159.2,138.5,136.4,130.3,128.8,113.4,77.3,77.2,77.0$, 76.7, 75.2, 66.1, 62.6, 60.6, 20.8, 20.7, 20.7. IR $\left(\mathrm{CHCl}_{3} \mathrm{~cm}^{-1}\right): v 1751,1620 ; \mathrm{HRMS}(\mathrm{ESI}+): \mathrm{m} / \mathrm{z}$ calcd. For $\mathrm{C}_{19} \mathrm{H}_{19} \mathrm{ClNaO}_{8}(\mathrm{M}+\mathrm{Na})^{+} 433.0666$, found 433.0671. $[\alpha]_{\mathrm{D}}+78.2\left(\mathrm{c} 1, \mathrm{CHCl}_{3}\right)$.

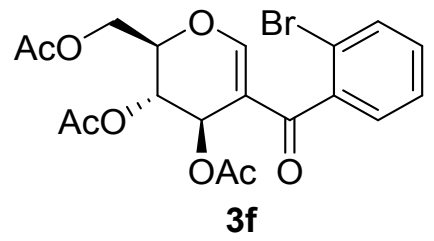

2-(acetoxymethyl)-5-(2-bromobenzoyl)-3,4-dihydro-2H-pyran-3,4-diyl diacetate (3f). Prepared according to general procedure 2.1. In an oven dried single neck round bottom flask charged with magnetic bead and flashed three times with $\mathrm{N}_{2}, 2$-iodo-tri- $O$-acetyl-D-glucal $(0.25$ $\mathrm{mmol}), \mathrm{Pd}(\mathrm{OAc})_{2}\left(0.012 \mathrm{mmol}, 0.05\right.$ equiv), $\mathrm{PPh}_{3}(0.025 \mathrm{mmol}, 0.1$ equiv) in $5 \mathrm{~mL}$ of dry toluene were added. In the same solution 2-bromo- phenyl boronic acid ( $0.3 \mathrm{mmol}, 1.2$ equiv), DCC ( 0.5 mmol, 2 equiv) and $\mathrm{K}_{2} \mathrm{CO}_{3}(0.5 \mathrm{mmol}, 2$ equiv) were also added and the reaction mixture was sealed with rubber septa. Formic acid ( $0.5 \mathrm{mmol}, 2$ equiv), triethyl amine ( $0.5 \mathrm{mmol}, 2$ equiv) were added via syringe sequentially and stirred the reaction mixture at $\mathrm{rt}$ under $\mathrm{N}_{2}$ atmosphere until complete consumption of starting material was observed by TLC analysis Then the reaction mixture was diluted with chilled $10 \mathrm{~mL}$ of toluene/hexane (1:1) to precipitate the DCU formed from DCC. This precipitation process was repeated at least three times before filtration through a filter paper. The filtrate was washed with $20 \mathrm{~mL}$ of ethyl acetate and brine solution. The residue left was purified by column chromatography over silica gel (60-120 mesh) using pet ether/ ethyl acetate (8:1.5) as eluent as yellow oil ( $60 \%, 69 \mathrm{mg}) .1 \mathrm{H} \mathrm{NMR} \mathrm{(400} \mathrm{MHz,} \mathrm{CDCl3)} \delta 7.60$ (d, $J=$ $8.1 \mathrm{~Hz}, 1 \mathrm{H}), 7.41-7.35$ (m, 1H), 7.30 (dd, $J=13.8,6.5 \mathrm{~Hz}, 2 \mathrm{H}), 7.21$ (s, 1H), 5.90 (d, $J=1.7 \mathrm{~Hz}$, $1 \mathrm{H}), 5.26(\mathrm{t}, J=3.2 \mathrm{~Hz}, 1 \mathrm{H}), 4.61(\mathrm{~d}, J=3.3 \mathrm{~Hz}, 1 \mathrm{H}), 4.51(\mathrm{dd}, J=12.0,7.6 \mathrm{~Hz}, 1 \mathrm{H}), 4.21(\mathrm{dd}, J$ $=12.0,4.1 \mathrm{~Hz}, 1 \mathrm{H}), 2.10(\mathrm{~s}, 6 \mathrm{H}), 2.07(\mathrm{~s}, 3 \mathrm{H}) .{ }^{13} \mathrm{C} \mathrm{NMR}\left(101 \mathrm{MHz}, \mathrm{CDCl}_{3}\right) \delta$ 192.3, 170.3, 169.5, 169.3, 161.1, 139.9, 133.3, 131.1, 128.70, 127.3, 119.6, 113.8, 75.5, 66.0, 61.8, 61.0, 20.8, 20.7, 
20.6. IR $\left(\mathrm{CHCl}_{3} \mathrm{~cm}^{-1}\right): v$ 1744, 1622; $\mathrm{HRMS}(\mathrm{ESI}+)$ : $\mathrm{m} / \mathrm{z}$ calcd. For $\mathrm{C}_{19} \mathrm{H}_{19} \mathrm{BrNaO}_{8}(\mathrm{M}+\mathrm{Na})^{+}$ 477.0161, found 477.0166. $[\alpha]_{\mathrm{D}}+92.2\left(\mathrm{c} 1, \mathrm{CHCl}_{3}\right)$.

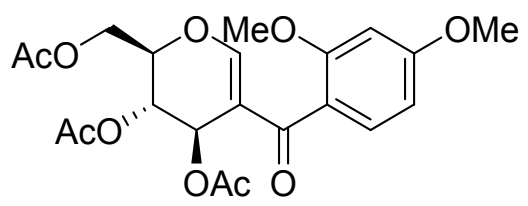

$3 \mathbf{g}$

2-(acetoxymethyl)-5-(2,4-dimethoxybenzoyl)-3,4-dihydro-2H-pyran-3,4-diyl diacetate (3g). Prepared according to general procedure 2.1. In an oven dried single neck round bottom flask charged with magnetic bead and flashed three times with $\mathrm{N}_{2}, 2$-iodo-tri- $O$-acetyl-D-glucal $(0.25$ $\mathrm{mmol}), \mathrm{Pd}(\mathrm{OAc})_{2}\left(0.012 \mathrm{mmol}, 0.05\right.$ equiv), $\mathrm{PPh}_{3}(0.025 \mathrm{mmol}, 0.1$ equiv) in $5 \mathrm{~mL}$ of dry toluene were added. In the same solution (2-bromo-5-methoxyphenyl)boronic acid (0.3 mmol, 1.2 equiv), DCC ( $0.5 \mathrm{mmol}, 2$ equiv) and $\mathrm{K}_{2} \mathrm{CO}_{3}(0.5 \mathrm{mmol}, 2$ equiv $)$ were also added and the reaction mixture was sealed with rubber septa. Formic acid ( $0.5 \mathrm{mmol}, 2$ equiv), triethyl amine ( $0.5 \mathrm{mmol}, 2$ equiv) were added via syringe sequentially and stirred the reaction mixture at rt under $\mathrm{N}_{2}$ atmosphere until complete consumption of starting material was observed by TLC analysis. Then the reaction mixture was diluted with chilled $10 \mathrm{~mL}$ of toluene/hexane $(1: 1)$ to precipitate the DCU formed from DCC. This precipitation process was repeated at least three times before filtration through a filter paper. The filtrate was washed with $20 \mathrm{~mL}$ of ethyl acetate and brine solution. The organic layer was dried over sodium sulphate and evaporated in vacuo. The residue left was purified by column chromatography over silica gel (60-120 mesh) using pet ether/ ethyl acetate (8:1.5) as eluent as yellow oil ( 57\%, $70 \mathrm{mg}) .1 \mathrm{H} \mathrm{NMR} \mathrm{(400} \mathrm{MHz,} \mathrm{CDCl3)} \delta 7.28$ (dd, $J=9.3,3.2 \mathrm{~Hz}, 3 \mathrm{H})$, $6.56-6.44(\mathrm{~m}, 2 \mathrm{H}), 5.93(\mathrm{~d}, J=3.5 \mathrm{~Hz}, 1 \mathrm{H}), 5.24(\mathrm{~d}, J=3.5 \mathrm{~Hz}, 1 \mathrm{H}), 4.54(\mathrm{~d}, J=8.3 \mathrm{~Hz}, 2 \mathrm{H})$, $4.17(\mathrm{~d}, J=8.1 \mathrm{~Hz}, 1 \mathrm{H}), 3.83(\mathrm{~s}, 6 \mathrm{H}), 2.10$ (s, 6H), 2.02 (s, 3H). 13C NMR (126 MHz, CDCl3) $\delta$ 191.8, 170.4, 169.7, 169.5, 162.9, 159.2, 158.8, 131.4, 121.0, 114.6, 104.5, 98.9, 74.9, 66.4, 62.7, 61.0, 55.8, 55.5, 20.9, 20.8. IR $\left(\mathrm{CHCl}_{3} \mathrm{~cm}^{-1}\right): v$ 1735, 1646; HRMS (ESI+): m/z calcd. For

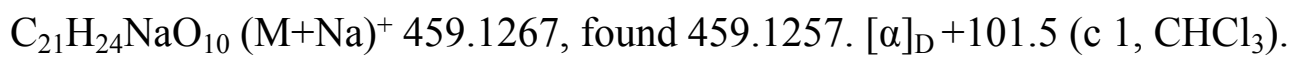<smiles>O=C(C1=COC(COc2ccccc2)[C@H](OCc2ccccc2)[C@@H]1OCc1ccccc1)c1ccccc1</smiles>

3h 


\section{3,4-bis(benzyloxy)-2-((benzyloxy)methyl)-3,4-dihydro-2H-pyran-5-yl)(phenyl)methanone}

(3h). Prepared according to general procedure 2.1. In an oven dried single neck round bottom flask charged with magnetic bead and flashed three times with $\mathrm{N}_{2}$, 2-iodo-tri-O-benzyl-D-glucal ( 0.18 $\mathrm{mmol}), \mathrm{Pd}(\mathrm{OAc})_{2}\left(0.009 \mathrm{mmol}, 0.05\right.$ equiv), $\mathrm{PPh}_{3}(0.018 \mathrm{mmol}, 0.1$ equiv) in $5 \mathrm{~mL}$ of dry toluene were added. In the same solution phenyl boronic acid $(0.21 \mathrm{mmol}, 1.2$ equiv), DCC ( $0.36 \mathrm{mmol}$, 2 equiv) and $\mathrm{K}_{2} \mathrm{CO}_{3}(0.36 \mathrm{mmol}, 2$ equiv) were also added and the reaction mixture was sealed with rubber septa. Formic acid ( $0.36 \mathrm{mmol}, 2$ equiv), triethyl amine $(0.36 \mathrm{mmol}, 2$ equiv) were added via syringe sequentially and stirred the reaction mixture at $\mathrm{rt}$ under Then the reaction mixture was diluted with chilled $10 \mathrm{~mL}$ of toluene/hexane (1:1) to precipitate the DCU formed from DCC. This precipitation process was repeated at least three times before filtration through a filter paper. The filtrate was washed with $20 \mathrm{~mL}$ of ethyl acetate and brine solution. The organic layer was dried over sodium sulphate and evaporated in vacuo. The residue left was purified by column chromatography over silica gel (60-120 mesh) using pet ether/ ethyl acetate (8:1) as eluent as yellow oil ( $73 \%, 70 \mathrm{mg}) .{ }^{1} \mathrm{H}$ NMR (400 MHz, $\left.\mathrm{CDCl}_{3}\right) \delta 7.65-7.60(\mathrm{~m}, 2 \mathrm{H}), 7.53(\mathrm{~m}, 1 \mathrm{H}), 7.44$ (m, 2H), $7.41-7.29$ (m, 15H), $4.81-4.77$ (m, 1H), 4.69 (d, $J=11.4 \mathrm{~Hz}, 2 \mathrm{H}), 4.57$ (m, 5H), 3.94 $-3.88(\mathrm{~m}, 2 \mathrm{H}), 3.69(\mathrm{dd}, J=10.8,4.4 \mathrm{~Hz}, 1 \mathrm{H}) .{ }^{13} \mathrm{C} \mathrm{NMR}\left(101 \mathrm{MHz}, \mathrm{CDCl}_{3}\right) \delta 195.3,159.5,138.9$, $138.4,137.8,137.5,135.2,131.4,130.2,128.9,128.5,128.5,128.3,128.3,128.0,127.8,127.7$, 115.1, 77.8, 73.3, 72.6, 71.8, 71.7, 68.3, 67.1. IR $\left(\mathrm{CHCl}_{3} \mathrm{~cm}^{-1}\right): v 1643$; HRMS (ESI+): $\mathrm{m} / \mathrm{z}$ calcd. For $\mathrm{C}_{34} \mathrm{H}_{32} \mathrm{NaO}_{5}(\mathrm{M}+\mathrm{Na})^{+} 543.2147$, found 543.2139. $[\alpha]_{\mathrm{D}}+45.7\left(\mathrm{c} 1, \mathrm{CHCl}_{3}\right)$.

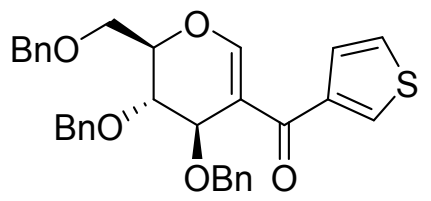

$3 \mathbf{i}$

\section{3,4-bis(benzyloxy)-2-((benzyloxy)methyl)-3,4-dihydro-2H-pyran-5-yl)(thiophen-3-}

yl)methanone (3i). Prepared according to general procedure 2.1. In an oven dried single neck round bottom flask charged with magnetic bead and flashed three times with $\mathrm{N}_{2}, 2$-iodo-tri- $O$ benzyl-D-glucal ( $0.18 \mathrm{mmol}), \mathrm{Pd}(\mathrm{OAc})_{2}\left(0.009 \mathrm{mmol}, 0.05\right.$ equiv), $\mathrm{PPh}_{3}(0.018 \mathrm{mmol}, 0.1$ equiv) in $5 \mathrm{~mL}$ of dry toluene were added. In the same solution 3-thienyl boronic acid $(0.21 \mathrm{mmol}, 1.2$ equiv), DCC ( $0.36 \mathrm{mmol}, 2$ equiv) and $\mathrm{K}_{2} \mathrm{CO}_{3}(0.36 \mathrm{mmol}, 2$ equiv) were also added and the reaction mixture was sealed with rubber septa. Formic acid $(0.36 \mathrm{mmol}, 2$ equiv), triethyl amine ( $0.36 \mathrm{mmol}, 2$ equiv) were added via syringe sequentially and stirred the reaction mixture at $\mathrm{rt}$ 
under $\mathrm{N}_{2}$ atmosphere until complete consumption of starting material was observed by TLC analysis. Then the reaction mixture was diluted with chilled $10 \mathrm{~mL}$ of toluene/hexane (1:1) to precipitate the DCU formed from DCC. This precipitation process was repeated at least three times before filtration through a filter paper. The filtrate was washed with $20 \mathrm{~mL}$ of ethyl acetate and brine solution. The organic layer was dried over sodium sulphate and evaporated in vacuo. The residue left was purified by column chromatography over silica gel (60-120 mesh) using pet ether/ ethyl acetate (9:1) as eluent as colorless oil ( 67\%, $85 \mathrm{mg}) .{ }^{1} \mathrm{H}$ NMR (400 $\left.\mathrm{MHz}, \mathrm{CDCl}_{3}\right) \delta 7.69$ $(\mathrm{dd}, J=2.8,1.2 \mathrm{~Hz}, 1 \mathrm{H}), 7.53(\mathrm{~s}, 1 \mathrm{H}), 7.42(\mathrm{dd}, J=5.0,1.2 \mathrm{~Hz}, 1 \mathrm{H}), 7.39-7.30(\mathrm{~m}, 9 \mathrm{H}), 7.26(\mathrm{~m}$, $7 \mathrm{H}), 4.79-4.75(\mathrm{~m}, 1 \mathrm{H}), 4.70-4.62(\mathrm{~m}, 2 \mathrm{H}), 4.59(\mathrm{~d}, J=5.9 \mathrm{~Hz}, 1 \mathrm{H}), 4.55(\mathrm{~d}, J=5.1 \mathrm{~Hz}, 3 \mathrm{H})$, $4.13(\mathrm{q}, J=7.2 \mathrm{~Hz}, 1 \mathrm{H}), 3.92-3.85(\mathrm{~m}, 2 \mathrm{H}), 3.68(\mathrm{dd}, J=10.8,4.4 \mathrm{~Hz}, 1 \mathrm{H}) .{ }^{13} \mathrm{C} \mathrm{NMR}(101 \mathrm{MHz}$, $\left.\mathrm{CDCl}_{3}\right) \delta 188.6,158.0,141.5,138.4,137.8,137.5,130.5,128.5,128.5,128.3,128.1,127.9,127.8$, 127.7, 126.1, 116.0, 77.8, 73.3, 72.6, 71.8, 71.7, 68.2, 67.2. IR $\left(\mathrm{CHCl}_{3} \mathrm{~cm}^{-1}\right): v 1645 ;$ HRMS $(\mathrm{ESI}+): \mathrm{m} / \mathrm{z}$ calcd. For $\mathrm{C}_{32} \mathrm{H}_{30} \mathrm{NaO}_{5} \mathrm{~S}(\mathrm{M}+\mathrm{Na})^{+} 549.1712$, found 549.1722. $[\alpha]_{\mathrm{D}}+66.4\left(\mathrm{c} 1, \mathrm{CHCl}_{3}\right)$.

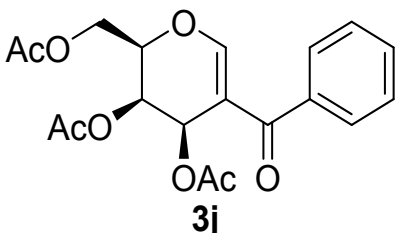

2-(acetoxymethyl)-5-benzoyl-3,4-dihydro-2H-pyran-3,4-diyl diacetate (3j). Prepared according to general procedure 2.1. In an oven dried single neck round bottom flask charged with magnetic bead and flashed three times with $\mathrm{N}_{2}, 2$-iodo-tri- $O$-acetyl-D-galactal $(0.25 \mathrm{mmol})$, $\mathrm{Pd}(\mathrm{OAc})_{2}\left(0.012 \mathrm{mmol}, 0.05\right.$ equiv), $\mathrm{PPh}_{3}(0.025 \mathrm{mmol}, 0.1$ equiv) in $5 \mathrm{~mL}$ of dry toluene were added. In the same solution phenyl boronic acid ( $0.3 \mathrm{mmol}, 1.2$ equiv), DCC ( $0.5 \mathrm{mmol}, 2$ equiv) and $\mathrm{K}_{2} \mathrm{CO}_{3}(0.5 \mathrm{mmol}, 2$ equiv) were also added and the reaction mixture was sealed with rubber septa. Formic acid ( $0.5 \mathrm{mmol}, 2$ equiv), triethyl amine ( $0.5 \mathrm{mmol}, 2$ equiv) were added via syringe sequentially and stirred the reaction mixture at $r$ under $\mathrm{N}_{2}$ atmosphere until complete consumption of starting material was observed by TLC analysis. Then the reaction mixture was diluted with chilled $10 \mathrm{~mL}$ of toluene/hexane $(1: 1)$ to precipitate the DCU formed from DCC. This precipitation process was repeated at least three times before filtration through a filter paper. The filtrate was washed with $20 \mathrm{~mL}$ of ethyl acetate and brine solution. The organic layer was dried over sodium sulphate and evaporated in vacuo. The residue left was purified by column chromatography over silica gel (60-120 mesh) using pet ether/ ethyl acetate (8:1.6) as eluent as yellow oil ( 71\%, 99 
mg). ${ }^{1} \mathrm{H}$ NMR (400 MHz, $\left.\mathrm{CDCl}_{3}\right) \delta 7.69-7.65(\mathrm{~m}, 2 \mathrm{H}), 7.57-7.51(\mathrm{~m}, 1 \mathrm{H}), 7.45$ (t, $J=7.4 \mathrm{~Hz}$, 2H), $7.33(\mathrm{~s}, 1 \mathrm{H}), 6.23(\mathrm{~d}, J=4.4 \mathrm{~Hz}, 1 \mathrm{H}), 5.52(\mathrm{dd}, J=4.3,2.8 \mathrm{~Hz}, 1 \mathrm{H}), 4.60-4.46(\mathrm{~m}, 2 \mathrm{H})$, $4.32-4.23(\mathrm{~m}, 1 \mathrm{H}), 2.15(\mathrm{~s}, 3 \mathrm{H}), 2.11(\mathrm{~s}, 3 \mathrm{H}), 2.00(\mathrm{~s}, 3 \mathrm{H}) .{ }^{13} \mathrm{C} \mathrm{NMR}\left(101 \mathrm{MHz}, \mathrm{CDCl}_{3}\right) \delta 192.2$, $170.5,169.9,169.6,158.5,138.2,132.0,128.8,128.5,114.4,74.3,64.5,61.7,61.3,20.7$, 20.6, 20.5. IR $\left(\mathrm{CHCl}_{3} \mathrm{~cm}^{-1}\right): v 1752,1645$; HRMS (ESI+): $\mathrm{m} / \mathrm{z}$ calcd. For $\mathrm{C}_{19} \mathrm{H}_{20} \mathrm{NaO}_{8}(\mathrm{M}+\mathrm{Na})^{+}$ 399.1056, found 399.1064. $[\alpha]_{\mathrm{D}}-66.4\left(\mathrm{c} 1, \mathrm{CHCl}_{3}\right)$.

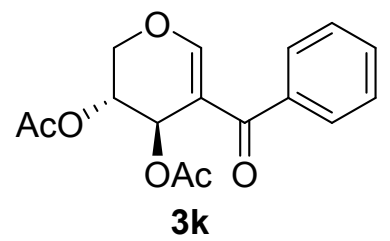

5-benzoyl-3,4-dihydro-2H-pyran-3,4-diyl diacetate (3k). Prepared according to general procedure 2.1. In an oven dried single neck round bottom flask charged with magnetic bead and flashed three times with $\mathrm{N}_{2}$, 2-iodo-di- $O$-acetyl-D-xylal $(0.30 \mathrm{mmol}), \mathrm{Pd}(\mathrm{OAc})_{2}(0.03 \mathrm{mmol}, 0.015$ equiv), $\mathrm{PPh}_{3}$ ( $0.03 \mathrm{mmol}, 0.1$ equiv) in $5 \mathrm{~mL}$ of dry toluene were added. In the same solution phenyl boronic acid (0.36 mmol, 1.2 equiv), DCC ( $0.36 \mathrm{mmol}, 2$ equiv) and $\mathrm{K}_{2} \mathrm{CO}_{3}(0.36 \mathrm{mmol}$, 2 equiv) were also added and the reaction mixture was sealed with rubber septa. Formic acid (0.36 mmol, 2 equiv), triethyl amine ( $0.36 \mathrm{mmol}, 2$ equiv) were added via syringe sequentially and stirred the reaction mixture at $\mathrm{rt}$ under $\mathrm{N}_{2}$ atmosphere until complete consumption of starting material was observed by TLC analysis. Then the reaction mixture was diluted with chilled $10 \mathrm{~mL}$ of toluene/hexane $(1: 1)$ to precipitate the DCU formed from DCC. This precipitation process was repeated at least three times before filtration through a filter paper. The filtrate was washed with $20 \mathrm{~mL}$ of ethyl acetate and brine solution. The organic layer was dried over sodium sulphate and evaporated in vacuo. The residue left was purified by column chromatography over silica gel (60$120 \mathrm{mesh})$ using pet ether/ ethyl acetate (8:1.2) as eluent as yellow oil (73\%, $111 \mathrm{mg})$. 1H NMR $(400 \mathrm{MHz}, \mathrm{CDCl} 3) \delta 7.63(\mathrm{~d}, J=7.2 \mathrm{~Hz}, 2 \mathrm{H}), 7.54-7.48(\mathrm{~m}, 2 \mathrm{H}), 7.43(\mathrm{t}, J=7.4 \mathrm{~Hz}, 2 \mathrm{H}), 5.88$ $(\mathrm{s}, 1 \mathrm{H}), 5.10(\mathrm{~s}, 1 \mathrm{H}), 4.46(\mathrm{~d}, J=12.5 \mathrm{~Hz}, 1 \mathrm{H}), 4.11(\mathrm{~d}, J=12.5 \mathrm{~Hz}, 1 \mathrm{H}), 2.07(\mathrm{~s}, 6 \mathrm{H}) .{ }^{13} \mathrm{C} \mathrm{NMR}$ $\left(101 \mathrm{MHz}, \mathrm{CDCl}_{3}\right) \delta 193.6,169.6,169.3,161.9,138.4,131.6,128.7,128.4,112.9,65.4,64.7$, 60.8, 21.0, 20.9. IR $\left(\mathrm{CHCl}_{3} \mathrm{~cm}^{-1}\right): v$ 1740, 1650; HRMS (ESI+): $\mathrm{m} / \mathrm{z}$ calcd. For $\mathrm{C}_{16} \mathrm{H}_{16} \mathrm{NaO}_{6}$ $(\mathrm{M}+\mathrm{Na})^{+}$327.0845, found 327.0853. $[\alpha]_{\mathrm{D}}+120.1\left(\mathrm{c} 1, \mathrm{CHCl}_{3}\right)$. 


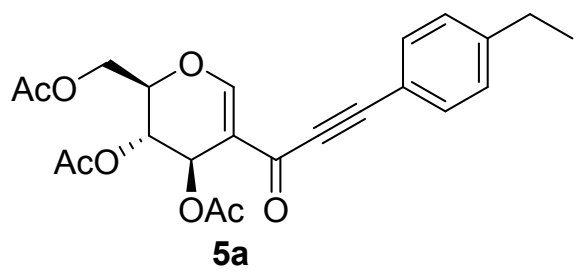

\section{2-(acetoxymethyl)-5-(3-(4-ethylphenyl) propioloyl)-3,4-dihydro-2H-pyran-3,4-diyl diacetate}

(5a). Prepared according to general procedure 2.2. In an oven dried single neck round bottom flask charged with magnetic bead and flashed three times with $\mathrm{N}_{2}, 2$-iodo-tri-O-acetyl-D-glucal (0.25 $\mathrm{mmol}), \mathrm{Pd}(\mathrm{OAc})_{2}\left(0.012 \mathrm{mmol}, 0.05\right.$ equiv), $\mathrm{PPh}_{3}(0.025 \mathrm{mmol}, 0.1$ equiv) in $5 \mathrm{~mL}$ of dry toluene were added. In the same solution 1-ethyl-4-ethynylbenzene $(0.3 \mathrm{mmol}, 1.2$ equiv), and DCC $(0.5$ mmol, 2 equiv) were also added and the reaction mixture was sealed with rubber septa. Formic acid ( $0.5 \mathrm{mmol}, 2$ equiv), triethyl amine $(0.5 \mathrm{mmol}, 2$ equiv) were added via syringe sequentially and stirred the reaction mixture at $\mathrm{rt}$ under $\mathrm{N}_{2}$ atmosphere until complete consumption of starting material was observed by TLC analysis. Then the reaction mixture was diluted with chilled $10 \mathrm{~mL}$ of toluene/hexane (1:1) to precipitate the DCU formed from DCC. This precipitation process was repeated at least three times before filtration through a filter paper. The filtrate was washed with $20 \mathrm{~mL}$ of ethyl acetate and brine solution. The organic layer was dried over sodium sulphate and evaporated in vacuo. The residue left was purified by column chromatography over silica gel (60120 mesh) using pet ether/ ethyl acetate (82:18) as eluent as yellow oil ( 68\%, $73 \mathrm{mg}) .{ }^{1} \mathrm{H}$ NMR $\left(400 \mathrm{MHz}, \mathrm{CDCl}_{3}\right) \delta 8.07(\mathrm{~s}, 1 \mathrm{H}), 7.51(\mathrm{~d}, J=8.1 \mathrm{~Hz}, 2 \mathrm{H}), 7.22(\mathrm{~d}, J=8.3 \mathrm{~Hz}, 2 \mathrm{H}), 5.81$ (dd, $J=$ 3.0, $1.7 \mathrm{~Hz}, 1 \mathrm{H}), 5.22$ (t, $J=2.9 \mathrm{~Hz}, 1 \mathrm{H}), 4.66(\mathrm{dd}, J=4.8,3.1 \mathrm{~Hz}, 1 \mathrm{H}), 4.47$ (dd, $J=12.1,7.9$ $\mathrm{Hz}, 1 \mathrm{H}), 4.20(\mathrm{dd}, J=12.2,4.5 \mathrm{~Hz}, 1 \mathrm{H}), 2.75-2.66(\mathrm{~m}, 2 \mathrm{H}), 2.11(\mathrm{~s}, 3 \mathrm{H}), 2.09(\mathrm{~s}, 3 \mathrm{H}), 2.06(\mathrm{~s}$, $3 \mathrm{H}), 1.26(\mathrm{t}, 3 \mathrm{H}) .{ }^{13} \mathrm{C} \mathrm{NMR}\left(126 \mathrm{MHz}, \mathrm{CDCl}_{3}\right) \delta 174.6,170.3,169.5,169.2,160.7,147.6,133.0$, 128.3, 117.0, 114.9, 91.6, 84.8, 75.6, 65.7, 61.2, 61.0, 29.0, 20.8, 20.7, 20.7, 15.1. IR $\left(\mathrm{CHCl}_{3} \mathrm{~cm}^{-}\right.$ $\left.{ }^{1}\right): v 1742,1639 ; \mathrm{HRMS}(\mathrm{ESI}+): \mathrm{m} / \mathrm{z}$ calcd. For $\mathrm{C}_{23} \mathrm{H}_{24} \mathrm{NaO}_{8}(\mathrm{M}+\mathrm{Na})^{+} 451.1369$, found 451.1376 . $[\alpha]_{\mathrm{D}}+87.1$ (c $\left.1, \mathrm{CHCl}_{3}\right)$.

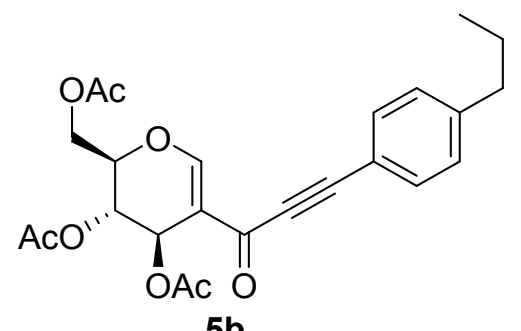

$5 b$ 


\section{2-(acetoxymethyl)-5-(3-(4-propylphenyl)propioloyl)-3,4-dihydro-2H-pyran-3,4-diyl}

diacetate $(\mathbf{5 b})$. Prepared according to general procedure 2.2. In an oven dried single neck round bottom flask charged with magnetic bead and flashed three times with $\mathrm{N}_{2}$, 2-iodo-tri- $O$-acetyl-Dglucal (0.25 mmol), $\mathrm{Pd}(\mathrm{OAc})_{2}\left(0.012 \mathrm{mmol}, 0.05\right.$ equiv), $\mathrm{PPh}_{3}(0.025 \mathrm{mmol}, 0.1$ equiv) in $5 \mathrm{~mL}$ of dry toluene were added. In the same solution 1-ethynyl-4-propylbenzene ( $0.3 \mathrm{mmol}, 1.2$ equiv), and DCC (0.5 mmol, 2 equiv) were also added and the reaction mixture was sealed with rubber septa. Formic acid ( $0.5 \mathrm{mmol}, 2$ equiv), triethyl amine ( $0.5 \mathrm{mmol}, 2$ equiv) were added via syringe sequentially and stirred the reaction mixture at $\mathrm{rt}$ under $\mathrm{N}_{2}$ atmosphere until complete consumption of starting material was observed by TLC analysis. Then the reaction mixture was diluted with chilled $10 \mathrm{~mL}$ of toluene/hexane $(1: 1)$ to precipitate the DCU formed from DCC. This precipitation process was repeated at least three times before filtration through a filter paper. The filtrate was washed with $20 \mathrm{~mL}$ of ethyl acetate and brine solution. The organic layer was dried over sodium sulphate and evaporated in vacuo. The residue left was purified by column chromatography over silica gel (60-120 mesh) using pet ether/ ethyl acetate (83:17) as eluent as yellow oil ( 67\%, 75 mg). 1H NMR (400 MHz, CDCl3) $\delta 8.08(\mathrm{~s}, 1 \mathrm{H}), 7.51$ (d, $J=8.1 \mathrm{~Hz}, 2 \mathrm{H}), 7.21(\mathrm{~d}, J=8.1 \mathrm{~Hz}$, 2H), $5.82(\mathrm{~s}, 1 \mathrm{H}), 5.24(\mathrm{~d}, J=2.8 \mathrm{~Hz}, 1 \mathrm{H}), 4.66(\mathrm{~d}, J=3.1 \mathrm{~Hz}, 1 \mathrm{H}), 4.48(\mathrm{dd}, J=12.1,7.9 \mathrm{~Hz}$, 1H), 4.21 (dd, $J=12.1,4.4 \mathrm{~Hz}, 1 \mathrm{H}), 2.62$ (t, $J=7.5 \mathrm{~Hz}, 2 \mathrm{H}), 2.12(\mathrm{~s}, 3 \mathrm{H}), 2.10(\mathrm{~s}, 3 \mathrm{H}), 2.06$ (s, $3 \mathrm{H}), 1.69-1.63(\mathrm{~m}, 2 \mathrm{H}), 0.95(\mathrm{t}, J=7.3 \mathrm{~Hz}, 3 \mathrm{H}) .{ }^{13} \mathrm{C} \mathrm{NMR}\left(126 \mathrm{MHz}, \mathrm{CDCl}_{3}\right) \delta 174.7,170.3$, $169.5,169.3,160.8,132.9,128.9,116.9,114.9,91.6,84.8,75.6,65.7,61.2,61.00,38.1,24.2,20.8$, 20.7, 20.7, 13.7. IR $\left(\mathrm{CHCl}_{3} \mathrm{~cm}^{-1}\right): v$ 1751, 1643; HRMS (ESI+): m/z calcd. For $\mathrm{C}_{24} \mathrm{H}_{26} \mathrm{NaO}_{8}$ $(\mathrm{M}+\mathrm{Na})^{+} 465.1525$, found 465.1520. $[\alpha]_{\mathrm{D}}+110.1\left(\mathrm{c} 1, \mathrm{CHCl}_{3}\right)$.

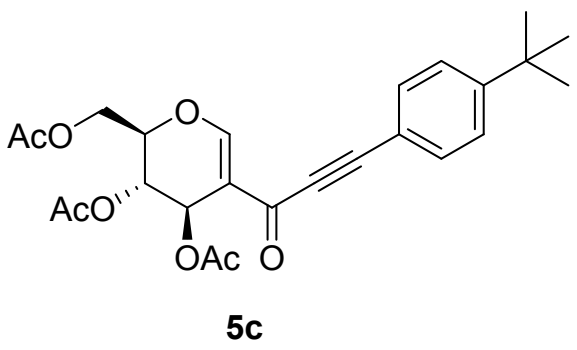

\section{2-(acetoxymethyl)-5-(3-(4-(tert-butyl)phenyl)propioloyl)-3,4-dihydro-2H-pyran-3,4-diyl}

diacetate (5c). Prepared according to general procedure 2.2. In an oven dried single neck round bottom flask charged with magnetic bead and flashed three times with $\mathrm{N}_{2}$, 2-iodo-tri- $O$-acetyl-Dglucal (0.25 mmol), $\mathrm{Pd}(\mathrm{OAc})_{2}\left(0.012 \mathrm{mmol}, 0.05\right.$ equiv), $\mathrm{PPh}_{3}(0.025 \mathrm{mmol}, 0.1$ equiv) in $5 \mathrm{~mL}$ 
of dry toluene were added. In the same solution 1-(tert-butyl)-4-ethynylbenzene (0.3 mmol, 1.2 equiv), and DCC (0.5 mmol, 2 equiv) were also added and the reaction mixture was sealed with rubber septa. Formic acid ( $0.5 \mathrm{mmol}, 2$ equiv), triethyl amine ( $0.5 \mathrm{mmol}, 2$ equiv) were added via syringe sequentially and stirred the reaction mixture at $\mathrm{rt}$ under $\mathrm{N}_{2}$ atmosphere until complete consumption of starting material was observed by TLC analysis. Then the reaction mixture was diluted with chilled $10 \mathrm{~mL}$ of toluene/hexane (1:1) to precipitate the DCU formed from DCC. This precipitation process was repeated at least three times before filtration through a filter paper. The filtrate was washed with $20 \mathrm{~mL}$ of ethyl acetate and brine solution. The organic layer was dried over sodium sulphate and evaporated in vacuo. The residue left was purified by column chromatography over silica gel (60-120 mesh) using pet ether/ ethyl acetate (85:15) as eluent as yellow oil ( 67\%, $77 \mathrm{mg}) .{ }^{1} \mathrm{H}$ NMR (400 MHz, $\left.\mathrm{CDCl}_{3}\right) \delta 8.07(\mathrm{~s}, 1 \mathrm{H}), 7.53(\mathrm{~d}, J=8.4 \mathrm{~Hz}, 2 \mathrm{H})$, $7.41(\mathrm{~d}, J=8.4 \mathrm{~Hz}, 2 \mathrm{H}), 5.82(\mathrm{dd}, J=2.9,1.7 \mathrm{~Hz}, 1 \mathrm{H}), 5.23(\mathrm{t}, J=3.0 \mathrm{~Hz}, 1 \mathrm{H}), 4.66(\mathrm{dd}, J=4.7$, $3.0 \mathrm{~Hz}, 1 \mathrm{H}), 4.47(\mathrm{dd}, J=12.3,7.9 \mathrm{~Hz}, 1 \mathrm{H}), 4.21(\mathrm{dd}, J=12.2,4.5 \mathrm{~Hz}, 1 \mathrm{H}), 2.12(\mathrm{~s}, 3 \mathrm{H}), 2.09$ (s, 3H), $2.06(\mathrm{~s}, 3 \mathrm{H}), 1.33(\mathrm{~s}, 9 \mathrm{H}) .{ }^{13} \mathrm{C} \mathrm{NMR}\left(101 \mathrm{MHz}, \mathrm{CDCl}_{3}\right) \delta 174.6,170.2,169.5,169.2,160.7$, 154.5, 132.8, 125.7, 116.8, 115.00, 91.5, 84.8, 75.6, 65.7, 61.4, 61.0, 31.0, 29.7, 20.7, 20.7, 20.6. IR $\left(\mathrm{CHCl}_{3} \mathrm{~cm}^{-1}\right): v$ 1750, 1644; HRMS (ESI+): m/z calcd. For $\mathrm{C}_{25} \mathrm{H}_{28} \mathrm{NaO}_{8}(\mathrm{M}+\mathrm{Na})^{+} 479.1682$, found 479.1683. $[\alpha]_{\mathrm{D}}+59.4\left(\mathrm{c} 1, \mathrm{CHCl}_{3}\right)$.



5d

\section{2-(acetoxymethyl)-5-(3-(4-fluorophenyl)propioloyl)-3,4-dihydro-2H-pyran-3,4-diyl}

diacetate (5d). Prepared according to general procedure 2.2. In an oven dried single neck round bottom flask charged with magnetic bead and flashed three times with $\mathrm{N}_{2}$, 2-iodo-tri- $O$-acetyl-Dglucal (0.25 mmol), $\mathrm{Pd}(\mathrm{OAc})_{2}\left(0.012 \mathrm{mmol}, 0.05\right.$ equiv), $\mathrm{PPh}_{3}(0.025 \mathrm{mmol}, 0.1$ equiv) in $5 \mathrm{~mL}$ of dry toluene were added. In the same solution 4-fluro-ethynylbenzene ( $0.3 \mathrm{mmol}, 1.2$ equiv), and DCC ( $0.5 \mathrm{mmol}, 2$ equiv) were also added and the reaction mixture was sealed with rubber septa. Formic acid ( $0.5 \mathrm{mmol}, 2$ equiv), triethyl amine $(0.5 \mathrm{mmol}, 2$ equiv) were added via syringe sequentially and stirred the reaction mixture at $\mathrm{rt}$ under $\mathrm{N}_{2}$ atmosphere until complete consumption of starting material was observed by TLC analysis. Then the reaction mixture was diluted with 
chilled $10 \mathrm{~mL}$ of toluene/hexane (1:1) to precipitate the DCU formed from DCC. This precipitation process was repeated at least three times before filtration through a filter paper. The filtrate was washed with $20 \mathrm{~mL}$ of ethyl acetate and brine solution. The organic layer was dried over sodium sulphate and evaporated in vacuo. The residue left was purified by column chromatography over silica gel (60-120 mesh) using pet ether/ ethyl acetate (8:1.8) as eluent as yellow oil ( $59 \%, 59$ mg). 1H NMR (400 MHz, CDCl3) $\delta 8.06(\mathrm{~s}, 1 \mathrm{H}), 7.60(\mathrm{dd}, J=8.3,5.4 \mathrm{~Hz}, 2 \mathrm{H}), 7.10(\mathrm{t}, J=8.5$ $\mathrm{Hz}, 2 \mathrm{H}), 5.81(\mathrm{~d}, J=1.5 \mathrm{~Hz}, 1 \mathrm{H}), 5.23(\mathrm{t}, J=2.8 \mathrm{~Hz}, 1 \mathrm{H}), 4.67(\mathrm{~d}, J=3.1 \mathrm{~Hz}, 1 \mathrm{H}), 4.49$ (dd, $J=$ 12.1, $7.9 \mathrm{~Hz}, 1 \mathrm{H}), 4.20(\mathrm{dd}, J=12.1,4.4 \mathrm{~Hz}, 1 \mathrm{H}), 2.12$ (s, 3H), $2.10(\mathrm{~s}, 3 \mathrm{H}), 2.06(\mathrm{~s}, 3 \mathrm{H}) .{ }^{13} \mathrm{C}$ NMR $\left(126 \mathrm{MHz}, \mathrm{CDCl}_{3}\right) \delta 174.5,170.3,169.5,169.2,160.9,135.2,135.1,116.3,116.2,115.9$, 114.8, 89.9, 84.9, 75.7, 65.6, 61.1, 60.9, 20.8, 20.7, 20.7. IR $\left(\mathrm{CHCl}_{3} \mathrm{~cm}^{-1}\right): v$ 1737, 1641; HRMS (ESI+): $\mathrm{m} / \mathrm{z}$ calcd. For $\mathrm{C}_{21} \mathrm{H}_{19} \mathrm{FNaO}_{8}(\mathrm{M}+\mathrm{Na})+441.0962$, found 441.0971. $[\alpha]_{\mathrm{D}}+96.7$ (c 1, $\left.\mathrm{CHCl}_{3}\right)$.

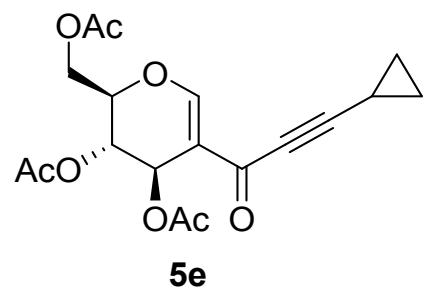

\section{2-(acetoxymethyl)-5-(3-cyclopropylpropioloyl)-3,4-dihydro-2H-pyran-3,4-diyl diacetate}

(5e). Prepared according to general procedure 2.2. In an oven dried single neck round bottom flask charged with magnetic bead and flashed three times with $\mathrm{N}_{2}$, 2-iodo-tri- $O$-acetyl-D-glucal $(0.25$ $\mathrm{mmol}), \mathrm{Pd}(\mathrm{OAc})_{2}\left(0.012 \mathrm{mmol}, 0.05\right.$ equiv), $\mathrm{PPh}_{3}(0.025 \mathrm{mmol}, 0.1$ equiv) in $5 \mathrm{~mL}$ of dry toluene were added. In the same solution ethynylcyclopropane ( $0.3 \mathrm{mmol}, 1.2$ equiv), and DCC $(0.5 \mathrm{mmol}$, 2 equiv) were also added and the reaction mixture was sealed with rubber septa. Formic acid $(0.5$ mmol, 2 equiv), triethyl amine ( $0.5 \mathrm{mmol}, 2$ equiv) were added via syringe sequentially and stirred the reaction mixture at $\mathrm{rt}$ under $\mathrm{N}_{2}$ atmosphere until complete consumption of starting material was observed by TLC analysis. Then the reaction mixture was diluted with chilled $10 \mathrm{~mL}$ of toluene/hexane (1:1) to precipitate the DCU formed from DCC. This precipitation process was repeated at least three times before filtration through a filter paper. The filtrate was washed with $20 \mathrm{~mL}$ of ethyl acetate and brine solution. The organic layer was dried over sodium sulphate and evaporated in vacuo. The residue left was purified by column chromatography over silica gel (60120 mesh) using pet ether/ ethyl acetate (8:1.8) as eluent as yellow oil ( $51 \%, 47 \mathrm{mg}) .{ }^{1} \mathrm{H}$ NMR 
$(400 \mathrm{MHz}, \mathrm{CDCl} 3) \delta 7.90(\mathrm{~s}, 1 \mathrm{H}), 5.70(\mathrm{dd}, J=2.8,1.7 \mathrm{~Hz}, 1 \mathrm{H}), 5.16(\mathrm{t}, J=2.9 \mathrm{~Hz}, 1 \mathrm{H}), 4.61$ $(\mathrm{dd}, J=4.7,3.2 \mathrm{~Hz}, 1 \mathrm{H}), 4.43(\mathrm{dd}, J=12.1,7.9 \mathrm{~Hz}, 1 \mathrm{H}), 4.15(\mathrm{dd}, J=12.3,4.4 \mathrm{~Hz}, 1 \mathrm{H}), 2.10$ (s, 3H), $2.06(\mathrm{~s}, 3 \mathrm{H}), 2.03(\mathrm{~s}, 3 \mathrm{H}), 1.45-1.37(\mathrm{~m}, 1 \mathrm{H}), 0.97(\mathrm{dt}, J=8.3,2.8 \mathrm{~Hz}, 2 \mathrm{H}), 0.92-0.88(\mathrm{~m}$, 2H). 13C NMR (101 MHz, CDCl3) $\delta 174.5,170.3,169.4,169.2,160.5,114.8,98.2,75.5,73.4$, 65.7, 61.2, 61.0, 20.8, 20.7 (2C), 9.5, 9.5, IR $\left(\mathrm{CHCl}_{3} \mathrm{~cm}^{-1}\right): v$ 1740, 1635; HRMS (ESI+): m/z calcd. For $\mathrm{C}_{18} \mathrm{H}_{20} \mathrm{NaO}_{8}(\mathrm{M}+\mathrm{Na})^{+}$387.1056, found 387.1045. $[\alpha]_{\mathrm{D}}+105.3$ (c 1, $\left.\mathrm{CHCl}_{3}\right)$.

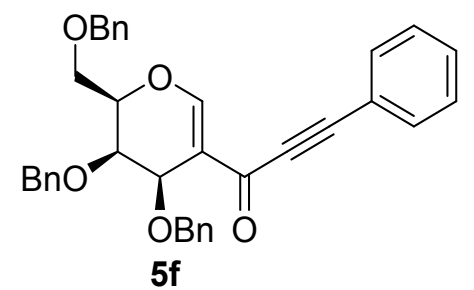

\section{3,4-bis(benzyloxy)-2-((benzyloxy)methyl)-3,4-dihydro-2H-pyran-5-yl)-3-phenylprop-2-yn-}

1-one (5f). Prepared according to general procedure 2.2. In an oven dried single neck round bottom flask charged with magnetic bead and flashed three times with $\mathrm{N}_{2}, 2$-iodo-tri- $O$-benzyl-D-galactal (0.18 mmol), $\mathrm{Pd}(\mathrm{OAc})_{2}\left(0.009 \mathrm{mmol}, 0.05\right.$ equiv), $\mathrm{PPh}_{3}(0.018 \mathrm{mmol}, 0.1$ equiv) in $5 \mathrm{~mL}$ of dry toluene were added. In the same solution phenyl acetylene $(0.21 \mathrm{mmol}, 1.2$ equiv), and DCC (0.36 mmol, 2 equiv) were also added and the reaction mixture was sealed with rubber septa. Formic acid ( $0.36 \mathrm{mmol}, 2$ equiv), triethyl amine $(0.36 \mathrm{mmol}, 2$ equiv $)$ were added via syringe sequentially and stirred the reaction mixture at $\mathrm{rt}$ under $\mathrm{N}_{2}$ atmosphere until complete consumption of starting material was observed by TLC analysis. Then the reaction mixture was diluted with chilled $10 \mathrm{~mL}$ of toluene/hexane (1:1) to precipitate the DCU formed from DCC. This precipitation process was repeated at least three times before filtration through a filter paper. The filtrate was washed with $20 \mathrm{~mL}$ of ethyl acetate and brine solution. The organic layer was dried over sodium sulphate and evaporated in vacuo. The residue left was purified by column chromatography over silica gel (60$120 \mathrm{mesh})$ using pet ether/ ethyl acetate (8:1.8) as eluent as yellow oil ( 63\%, $63 \mathrm{mg}) .{ }^{1} \mathrm{H}$ NMR $\left(400 \mathrm{MHz}, \mathrm{CDCl}_{3}\right) \delta 8.12(\mathrm{~s}, 1 \mathrm{H}), 7.59(\mathrm{~m}, 2 \mathrm{H}), 7.41(\mathrm{~m}, 3 \mathrm{H}), 7.38-7.33(\mathrm{~m}, 6 \mathrm{H}), 7.29(\mathrm{~m}, 9 \mathrm{H})$, $4.76-4.69(\mathrm{~m}, 2 \mathrm{H}), 4.59(\mathrm{~m}, 4 \mathrm{H}), 4.54-4.48(\mathrm{~m}, 2 \mathrm{H}), 3.88-3.81(\mathrm{~m}, 2 \mathrm{H}), 3.66(\mathrm{dd}, J=10.7$, 4.7 Hz, 1H). ${ }^{13} \mathrm{C}$ NMR $\left(101 \mathrm{MHz}, \mathrm{CDCl}_{3}\right) \delta 176.6,161.7,138.3,137.8,137.4,132.7,130.3,128.6$, 128.6, 128.5, 128.4, 128.1, 127.8, 127.8, 127.7, 120.4, 117.5, 90.1, 85.4, 78.7, 73.4, 72.7, 71.6, 71.4, 68.6, 65.9. IR $\left(\mathrm{CHCl}_{3} \mathrm{~cm}^{-1}\right): v$ 1648; HRMS (ESI+): $\mathrm{m} / \mathrm{z}$ calcd. For $\mathrm{C}_{36} \mathrm{H}_{32} \mathrm{NaO}_{5}(\mathrm{M}+\mathrm{Na})+$ 567.2147, found 567.2156. $[\alpha]_{\mathrm{D}}-49.3\left(\mathrm{c} 1, \mathrm{CHCl}_{3}\right)$. 


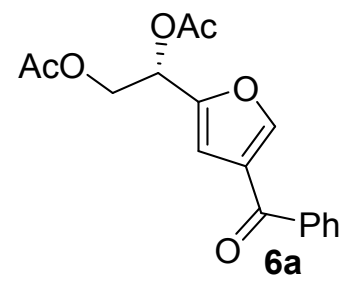

1-(4-benzoylfuran-2-yl) ethane-1,2-diyl diacetate (6a). Prepared according to procedure 2.3. Colorless liquid, (26.5 mg, 63\%), Purified by column chromatography over silica gel (60-120 mesh) using pet ether/ ethyl acetate (95:5). 1H NMR (400 MHz, CDCl3) $\delta 7.81$ (s, 1H), $7.79-$ $7.75(\mathrm{~m}, 2 \mathrm{H}), 7.53$ (t, $J=7.4 \mathrm{~Hz}, 1 \mathrm{H}), 7.43$ (t, $J=7.5 \mathrm{~Hz}, 2 \mathrm{H}), 6.84$ (s, 1H), 6.05 (dd, $J=7.2,4.4$ $\mathrm{Hz}, 1 \mathrm{H}), 4.46(\mathrm{dd}, J=11.7,4.6 \mathrm{~Hz}, 1 \mathrm{H}), 4.36(\mathrm{dd}, J=11.8,7.2 \mathrm{~Hz}, 1 \mathrm{H}), 2.06(\mathrm{~s}, 3 \mathrm{H}), 2.01(\mathrm{~s}, 3 \mathrm{H})$. ${ }^{13} \mathrm{C}$ NMR $\left(101 \mathrm{MHz}, \mathrm{CDCl}_{3}\right) \delta 189.0,170.5,169.8,151.2,148.4,138.5,132.7,128.8,128.6$, 127.8, 127.2, 109.7, 66.1, 63.0, 20.8, 20.7. IR $\left(\mathrm{CHCl}_{3} \mathrm{~cm}^{-1}\right): v$ 1746, 1649; HRMS (ESI+): m/z calcd. For $\mathrm{C}_{17} \mathrm{H}_{16} \mathrm{NaO}_{6}(\mathrm{M}+\mathrm{Na})^{+} 339.0845$, found 339.0855. $[\alpha]_{\mathrm{D}}+64.5$ (c 1, $\left.\mathrm{CHCl}_{3}\right)$.

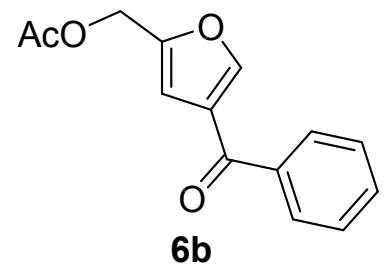

(4-benzoylfuran-2-yl) methyl acetate Prepared according to procedure 2.3. Colorless liquid, (23.5 mg, 59\%). Purified by column chromatography over silica gel (60-120 mesh) using pet ether/ ethyl acetate (95:5). ${ }^{1} \mathrm{H}$ NMR $\left(400 \mathrm{MHz} \mathrm{CDCl}_{3}\right) \delta 7.90(\mathrm{~s}, 1 \mathrm{H}), 7.85(\mathrm{~d}, J=7.9 \mathrm{~Hz}, 2 \mathrm{H})$, $7.60(\mathrm{t}, J=7.2 \mathrm{~Hz}, 1 \mathrm{H}), 7.50(\mathrm{~m}, 2 \mathrm{H}), 6.91(\mathrm{~s}, 1 \mathrm{H}), 5.11(\mathrm{~s}, 2 \mathrm{H}), 2.12(\mathrm{~s}, 3 \mathrm{H}) .{ }^{13} \mathrm{C}$ NMR $(126$ $\left.\mathrm{MHz}, \mathrm{CDCl}_{3}\right) \delta 189.2,170.5,151.2,148.8,138.5,132.7,128.9,128.7,110.8,57.7,20.83 . v$ 1743, 1647; HRMS (ESI+): m/z calcd. For $\mathrm{C}_{14} \mathrm{H}_{12} \mathrm{NaO}_{4}(\mathrm{M}+\mathrm{Na})^{+} 267.0633$, found 267.0637 . $[\alpha]_{\mathrm{D}}+47.4\left(\mathrm{c} 1, \mathrm{CHCl}_{3}\right)$.

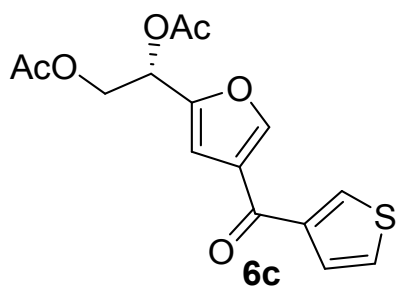


1-(4-(thiophene-3-carbonyl) furan-2-yl) ethane-1,2-diyl diacetate (6c). Prepared according to procedure 2.3. Colorless liquid, $(28.5 \mathrm{mg}, 67 \%)$. Purified by column chromatography over silica gel (60-120 mesh) using pet ether/ ethyl acetate (95:5).1H NMR (400 MHz, $\left.\mathrm{CDCl}_{3}\right) \delta 7.95$ (dd, $J$ $=2.8,1.1 \mathrm{~Hz}, 1 \mathrm{H}), 7.90(\mathrm{~s}, 1 \mathrm{H}), 7.50(\mathrm{dd}, J=5.0,1.0 \mathrm{~Hz}, 1 \mathrm{H}), 7.32(\mathrm{dd}, J=5.0,2.9 \mathrm{~Hz}, 1 \mathrm{H}), 6.82$ (s, 1H), $6.04(\mathrm{dd}, J=7.2,4.6 \mathrm{~Hz}, 1 \mathrm{H}), 4.45(\mathrm{dd}, J=11.7,4.6 \mathrm{~Hz}, 1 \mathrm{H}), 4.36(\mathrm{dd}, J=11.8,7.2 \mathrm{~Hz}$, 1H), $2.05(\mathrm{~s}, 3 \mathrm{H}), 2.00(\mathrm{~s}, 3 \mathrm{H}) .{ }^{13} \mathrm{C} \mathrm{NMR}\left(126 \mathrm{MHz}, \mathrm{CDCl}_{3}\right) \delta 182.1,170.5,169.9,151.2,147.4$, 142.0, 132.2, 127.9, 127.7, 126.8, 109.5, 66.0, 63.0, 20.9, 20.8. v 1735, 1637; HRMS (ESI+): m/z calcd. For $\mathrm{C}_{15} \mathrm{H}_{14} \mathrm{NaO}_{6} \mathrm{~S}(\mathrm{M}+\mathrm{Na})^{+} 345.0409$, found 345.0414. $[\alpha]_{\mathrm{D}}+65.6\left(\mathrm{c} 1, \mathrm{CHCl}_{3}\right)$.

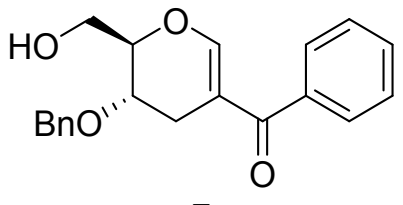

7

3-(benzyloxy)-2-(hydroxymethyl)-3,4-dihydro-2H-pyran-5-yl)(phenyl)methanone

(7).

Compound 7 was synthesized according to procedure 2.4. ${ }^{1} \mathrm{H} \mathrm{NMR}\left(400 \mathrm{MHz}, \mathrm{CDCl}_{3}\right) \delta 7.61-$ $7.57(\mathrm{~m}, 2 \mathrm{H}), 7.54-7.49(\mathrm{~m}, 1 \mathrm{H}), 7.46-7.38(\mathrm{~m}, 6 \mathrm{H}), 7.38-7.33(\mathrm{~m}, 1 \mathrm{H}), 7.31(\mathrm{~s}, 1 \mathrm{H}), 4.83(\mathrm{~d}$, $J=11.6 \mathrm{~Hz}, 1 \mathrm{H}), 4.62(\mathrm{~d}, J=11.7 \mathrm{~Hz}, 1 \mathrm{H}), 4.05-3.90(\mathrm{~m}, 3 \mathrm{H}), 3.83(\mathrm{td}, J=8.5,5.4 \mathrm{~Hz}, 1 \mathrm{H})$, $3.15(\mathrm{dd}, J=16.3,5.3 \mathrm{~Hz}, 1 \mathrm{H}), 2.42$ (ddd, $J=16.3,8.9,1.7 \mathrm{~Hz}, 1 \mathrm{H}) .{ }^{13} \mathrm{C} \mathrm{NMR}\left(101 \mathrm{MHz}, \mathrm{CDCl}_{3}\right)$ $\delta 195.29,159.28,139.13,137.61,131.13,128.61,128.25,128.07,127.95,114.29,79.25,70.94$, 69.47, 61.84, 25.30. IR $\left(\mathrm{CHCl}_{3} \mathrm{~cm}^{-1}\right): v$ 1642; HRMS (ESI+): m/z calcd. For $\mathrm{C}_{20} \mathrm{H}_{20} \mathrm{NaO}_{4}$ $(\mathrm{M}+\mathrm{Na})^{+}$347.1259, found 347.1259. $[\alpha]_{\mathrm{D}}+78.4\left(\mathrm{c} 1, \mathrm{CHCl}_{3}\right)$.

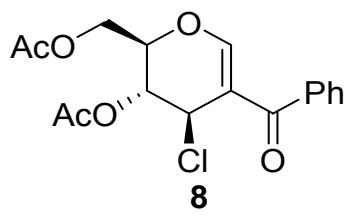

3-acetoxy-5-benzoyl-4-chloro-3,4-dihydro-2H-pyran-2-yl)methyl acetate (8). Compound 8 was synthesized according to procedure 2.5. 1H NMR (400 MHz, CDCl3) $\delta 7.54(\mathrm{~d}, J=7.7 \mathrm{~Hz}$, 2H), $7.45(\mathrm{dd}, J=10.8,3.9 \mathrm{~Hz}, 1 \mathrm{H}), 7.36(\mathrm{t}, J=7.7 \mathrm{~Hz}, 2 \mathrm{H}), 7.22(\mathrm{~s}, 1 \mathrm{H}), 5.51$ (d, $J=3.9 \mathrm{~Hz}$, $1 \mathrm{H}), 5.11(\mathrm{dd}, J=10.8,3.9 \mathrm{~Hz}, 1 \mathrm{H}), 4.63(\mathrm{dt}, J=10.8,2.8 \mathrm{~Hz}, 1 \mathrm{H}), 4.37(\mathrm{~d}, J=3.1 \mathrm{~Hz}, 2 \mathrm{H}), 2.11$ (s, 3H), 2.00 (s, 3H). ${ }^{13} \mathrm{C}$ NMR (101 MHz, $\left.\mathrm{CDCl}_{3}\right) \delta 191.7,170.4,169.3,159.2,138.2,131.9$, 128.7, 128.5, 116.6, 71.8, 66.1, 61.2, 49.7, 20.6, 20.6. IR $\left(\mathrm{CHCl}_{3} \mathrm{~cm}^{-1}\right): v 1748,1639$; HRMS 
(ESI+): $\mathrm{m} / \mathrm{z}$ calcd. For $\mathrm{C}_{17} \mathrm{H}_{17} \mathrm{ClNaO}_{6}(\mathrm{M}+\mathrm{Na})^{+}$375.0611, found 375.0625. $[\alpha]_{\mathrm{D}}+85.2$ (c 1, $\left.\mathrm{CHCl}_{3}\right)$.

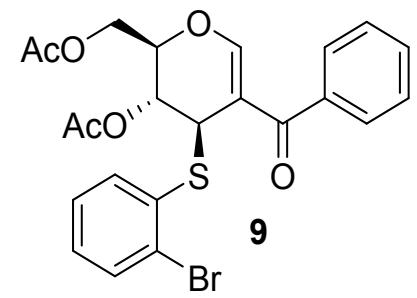

3-acetoxy-5-benzoyl-4-((2-bromophenyl) thio)-3,4-dihydro-2H-pyran-2-yl)methyl acetate (9). Compound 9 was synthesized according to procedure 2.5. ${ }^{1} \mathrm{H}$ NMR $\left(400 \mathrm{MHz}, \mathrm{CDCl}_{3}\right) \delta 7.85$ (dd, $J=8.0,1.4 \mathrm{~Hz}, 1 \mathrm{H}), 7.68-7.63(\mathrm{~m}, 2 \mathrm{H}), 7.59-7.53$ (m, 2H), $7.46(\mathrm{t}, J=7.6 \mathrm{~Hz}, 2 \mathrm{H}), 7.35$ $-7.30(\mathrm{~m}, 2 \mathrm{H}), 7.09(\mathrm{td}, J=7.9,1.3 \mathrm{~Hz}, 1 \mathrm{H}), 5.20(\mathrm{dd}, J=8.2,3.8 \mathrm{~Hz}, 2 \mathrm{H}), 4.92(\mathrm{dd}, J=7.5,3.1$ $\mathrm{Hz}, 1 \mathrm{H}), 4.47$ (d, J=3.1 Hz, 2H), 2.08 (s, 3H), $1.56(\mathrm{~s}, 3 \mathrm{H}) .{ }^{13} \mathrm{C} \mathrm{NMR}\left(101 \mathrm{MHz}, \mathrm{CDCl}_{3}\right) \delta 192.36$, $170.47,169.50,159.16,138.44,136.94,133.59,132.97,131.83,128.86,128.44,128.41,128.23$, 125.84, 114.14, 72.76, 67.16, 61.49, 43.10, 20.66, 19.76. IR $\left(\mathrm{CHCl}_{3} \mathrm{~cm}^{-1}\right): v 1743,1628$; HRMS (ESI+): $\mathrm{m} / \mathrm{z}$ calcd. For $\mathrm{C}_{23} \mathrm{H}_{22}{ }^{81} \mathrm{BrO}_{6}(\mathrm{M}+\mathrm{H})^{+}$507.0295, found 507.0286. $[\alpha]_{\mathrm{D}}+67.6\left(\mathrm{c} 1, \mathrm{CHCl}_{3}\right)$.

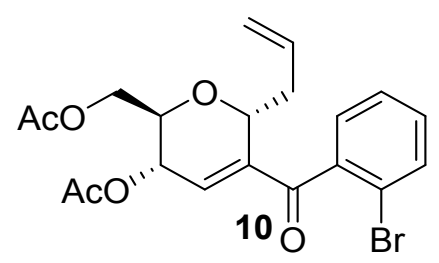

3-acetoxy-6-allyl-5-(2-bromobenzoyl)-3, 6-dihydro-2H-pyran-2-yl) methyl acetate (10). Compound 10 was synthesized according to procedure 2.5. 1H NMR (400 MHz, CDCl3) $\delta 7.62-$ $7.55(\mathrm{~m}, 1 \mathrm{H}), 7.41-7.27(\mathrm{~m}, 2 \mathrm{H}), 7.23(\mathrm{dd}, J=7.4,1.7 \mathrm{~Hz}, 1 \mathrm{H}), 6.23(\mathrm{~d}, J=1.1 \mathrm{~Hz}, 1 \mathrm{H}), 6.04-$ $5.88(\mathrm{~m}, 1 \mathrm{H}), 5.44-5.32(\mathrm{~m}, 1 \mathrm{H}), 5.14(\mathrm{~m}, 2 \mathrm{H}), 4.87(\mathrm{~d}, J=9.7 \mathrm{~Hz}, 1 \mathrm{H}), 4.22-4.15(\mathrm{~m}, 2 \mathrm{H})$, 4.03 (dt, $J=8.6,4.2 \mathrm{~Hz}, 1 \mathrm{H}), 2.67$ (m, 2H), 2.07 (s, 3H), 2.05 (s, 3H). 13C NMR (126 MHz, CDC13) $\delta 194.5,170.8,170.1,142.8,140.2,139.31,134.5,133.3,131.5,128.8,127.3,119.3$, 117.4, 71.9, 67.2, 64.9, 63.2, 35.9, 20.9, 20.8. IR $\left(\mathrm{CHCl}_{3} \mathrm{~cm}^{-1}\right): v$ 1737, 1646; HRMS (ESI+): m/z calcd. For $\mathrm{C}_{20} \mathrm{H}_{21} \mathrm{BrNaO} 6(\mathrm{M}+\mathrm{Na})^{+} 459.0419$, found 459.0413. $[\alpha]_{\mathrm{D}}+102.3$ (c 1, $\mathrm{CHCl}_{3}$ ). 


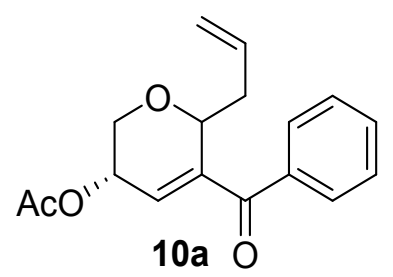

6-allyl-5-benzoyl-3, 6-dihydro-2H-pyran-3-yl acetate (10a). Compound 10a was synthesized according to procedure 2.5 colorless liquid (67\%). ${ }^{1} \mathrm{H}$ NMR $\left(400 \mathrm{MHz}, \mathrm{CDCl}_{3}\right) \delta 7.85-7.72(\mathrm{~m}$, 2H), $7.58(\mathrm{t}, J=7.4 \mathrm{~Hz}, 1 \mathrm{H}), 7.46(\mathrm{t}, J=7.7 \mathrm{~Hz}, 2 \mathrm{H}), 6.34(\mathrm{~s}, 1 \mathrm{H}), 5.95-5.79(\mathrm{~m}, 1 \mathrm{H}), 5.52$ $(\mathrm{ddd}, J=9.9,5.3,2.5 \mathrm{~Hz}, 1 \mathrm{H}), 5.04$ (t, $J=14.2 \mathrm{~Hz}, 2 \mathrm{H}), 4.96-4.84$ (m, 1H), 4.22 (dd, $J=11.2$, $5.5 \mathrm{~Hz}, 1 \mathrm{H}), 3.49$ (dd, $J=11.2,7.3 \mathrm{~Hz}, 1 \mathrm{H}), 2.58-2.41(\mathrm{~m}, 2 \mathrm{H}), 2.03(\mathrm{~s}, 3 \mathrm{H}) .{ }^{13} \mathrm{C}$ NMR (101 $\left.\mathrm{MHz}, \mathrm{CDCl}_{3}\right) \delta 195.9,170.2,143.5,136.8,135.9,133.7,132.9,129.7,128.4,118.0,73.8,64.5$, 64.4, 36.7, 20.9. $v$ 1743, 1648; HRMS (ESI+): $\mathrm{m} / \mathrm{z}$ calcd. For $\mathrm{C}_{17} \mathrm{H}_{18} \mathrm{NaO}_{4}(\mathrm{M}+\mathrm{Na})^{+} 309.1103$, found 309.1109. $[\alpha]_{\mathrm{D}}+86.5\left(\mathrm{c} 1, \mathrm{CHCl}_{3}\right)$.

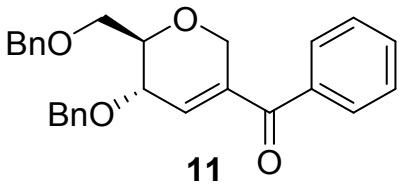

5-(benzyloxy)-6-((benzyloxy)methyl)-5,6-dihydro-2H-pyran-3-yl)(phenyl)methanone (11). Compound 11 was synthesized according to procedure $2.5{ }^{1} \mathrm{H} \mathrm{NMR}\left(400 \mathrm{MHz}, \mathrm{CDCl}_{3}\right) \delta 7.71$ (d, $J=7.2 \mathrm{~Hz}, 2 \mathrm{H}), 7.59$ (t, $J=7.3 \mathrm{~Hz}, 1 \mathrm{H}), 7.48(\mathrm{t}, J=7.6 \mathrm{~Hz}, 2 \mathrm{H}), 7.43-7.36(\mathrm{~m}, 4 \mathrm{H}), 7.35-7.30$ (m, 4H), $7.27-7.22(\mathrm{~m}, 2 \mathrm{H}), 6.59(\mathrm{~s}, 1 \mathrm{H}), 4.70(\mathrm{dd}, J=12.7,8.8 \mathrm{~Hz}, 2 \mathrm{H}), 4.62(\mathrm{~d}, J=3.9 \mathrm{~Hz}$, $1 \mathrm{H}), 4.59(\mathrm{~d}, J=4.6 \mathrm{~Hz}, 2 \mathrm{H}), 4.56-4.52(\mathrm{~m}, 1 \mathrm{H}), 4.41-4.36(\mathrm{~m}, 1 \mathrm{H}), 3.82(\mathrm{dd}, J=10.5,2.1 \mathrm{~Hz}$, 1H), $3.76(\mathrm{dd}, J=10.6,4.4 \mathrm{~Hz}, 1 \mathrm{H}), 3.67-3.61(\mathrm{~m}, 1 \mathrm{H}) .{ }^{13} \mathrm{C} \mathrm{NMR}\left(101 \mathrm{MHz}, \mathrm{CDCl}_{3}\right) \delta 195.3$, 139.0, 138.5, 138.0, 137.6, 137.2, 132.3, 129.2, 128.6, 128.5, 128.4, 128.4, 128.0, 128.0, 128.0, 127.8, 76.2, 73.6, 72.0, 70.4, 69.1, 65.7. IR $\left(\mathrm{CHCl}_{3} \mathrm{~cm}^{-1}\right): v$ 1730, 1643; HRMS (ESI+): m/z calcd. For $\mathrm{C}_{27} \mathrm{H}_{26} \mathrm{NaO}_{4}(\mathrm{M}+\mathrm{Na})^{+} 437.1729$, found 437.1736. $[\alpha]_{\mathrm{D}}+49.8\left(\mathrm{c} 1, \mathrm{CHCl}_{3}\right)$. 


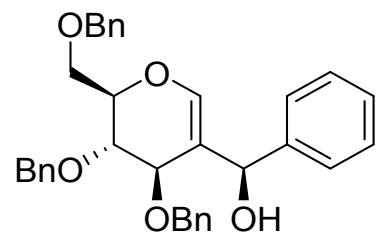

$12 a$

\section{3,4-bis(benzyloxy)-2-((benzyloxy)methyl)-3,4-dihydro-2H-pyran-5-yl)(phenyl)methanol}

(12a). Compound 12a was synthesized according to procedure 2.6. ${ }^{1} \mathrm{H} \mathrm{NMR}\left(400 \mathrm{MHz}, \mathrm{CDCl}_{3}\right)$ $\delta 7.35(\mathrm{~m}, 16 \mathrm{H}), 7.20(\mathrm{~m}, 4 \mathrm{H}), 6.63(\mathrm{~s}, 1 \mathrm{H}), 5.25(\mathrm{~s}, 1 \mathrm{H}), 4.59(\mathrm{~s}, 4 \mathrm{H}), 4.53(\mathrm{~d}, J=11.0 \mathrm{~Hz}, 1 \mathrm{H})$, $4.28(\mathrm{~s}, 1 \mathrm{H}), 4.19-4.14(\mathrm{~m}, 1 \mathrm{H}), 4.02-3.97(\mathrm{~m}, 2 \mathrm{H}), 3.86(\mathrm{dd}, J=10.5,5.6 \mathrm{~Hz}, 1 \mathrm{H}), 3.77(\mathrm{dd}, J$ $=10.5,4.2 \mathrm{~Hz}, 1 \mathrm{H}), 2.08(\mathrm{~s}, 1 \mathrm{H}) .{ }^{13} \mathrm{C} \mathrm{NMR}\left(126 \mathrm{MHz}, \mathrm{CDCl}_{3}\right) \delta 144.2,142.8,137.9,137.6,137.6$, 128.6, 128.5, 128.5, 128.2, 128.0, 128.0, 128.0, 127.9, 127.8, 126.9, 125.8, 113.7, 76.5, 74.2, 74.2, 73.8, 73.5, 72.9, 72.6, 67.9. IR $\left(\mathrm{CHCl}_{3} \mathrm{~cm}^{-1}\right): v$ 1747, 1649; HRMS (ESI+): m/z calcd. For $\mathrm{C}_{34} \mathrm{H}_{34} \mathrm{NaO}_{5}(\mathrm{M}+\mathrm{Na})^{+}$545.2304, found 545.2310. $[\alpha]_{\mathrm{D}}+68.9\left(\mathrm{c} 1, \mathrm{CHCl}_{3}\right)$.<smiles>O[C@@H]1C(COc2ccccc2)OC=C([C@H](O)c2ccccc2)[C@@H]1OCc1ccccc1</smiles>

$12 b$

\section{3,4-bis(benzyloxy)-2-((benzyloxy)methyl)-3,4-dihydro-2H-pyran-5-yl)(phenyl)methanol}

(12b). Compound 12b was synthesized according to procedure 2.6. ${ }^{1} \mathrm{H}$ NMR $\left(400 \mathrm{MHz}, \mathrm{CDCl}_{3}\right)$ $\delta 7.39-7.28(\mathrm{~m}, 18 \mathrm{H}), 7.20(\mathrm{dd}, J=7.0,2.4 \mathrm{~Hz}, 2 \mathrm{H}), 6.38(\mathrm{~s}, 1 \mathrm{H}), 5.34(\mathrm{~s}, 1 \mathrm{H}), 4.65(\mathrm{~s}, 2 \mathrm{H}), 4.55$ $(\mathrm{t}, J=5.5 \mathrm{~Hz}, 3 \mathrm{H}), 4.34(\mathrm{~m}, 2 \mathrm{H}), 4.04-3.96(\mathrm{~m}, 2 \mathrm{H}), 3.82(\mathrm{dd}, J=10.5,6.1 \mathrm{~Hz}, 1 \mathrm{H}), 3.72(\mathrm{dd}, J$ $=10.5,4.6 \mathrm{~Hz}, 1 \mathrm{H}) .{ }^{13} \mathrm{C} \mathrm{NMR}\left(101 \mathrm{MHz}, \mathrm{CDCl}_{3}\right) \delta 143.2,141.7,138.0,137.8,128.6,128.5$, $128.4,128.3,128.0,127.9,127.8,127.7,127.6,127.1,114.4,75.8,74.7,73.4,73.1,72.5,72.0$, 68.0. IR $\left(\mathrm{CHCl}_{3} \mathrm{~cm}^{-1}\right): v$ 1748, 1646; HRMS (ESI+): m/z calcd. For $\mathrm{C}_{34} \mathrm{H}_{34} \mathrm{NaO}_{5}(\mathrm{M}+\mathrm{Na})^{+}$ 545.2304, found 545.2308. $[\alpha]_{\mathrm{D}}+60.3\left(\mathrm{c} 1, \mathrm{CHCl}_{3}\right)$. 
NMR Spectra 


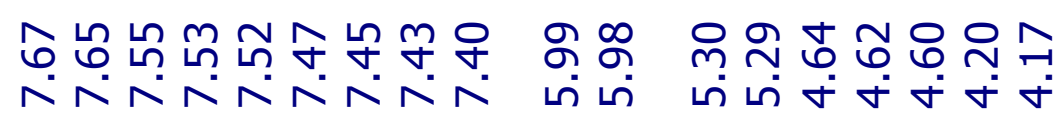

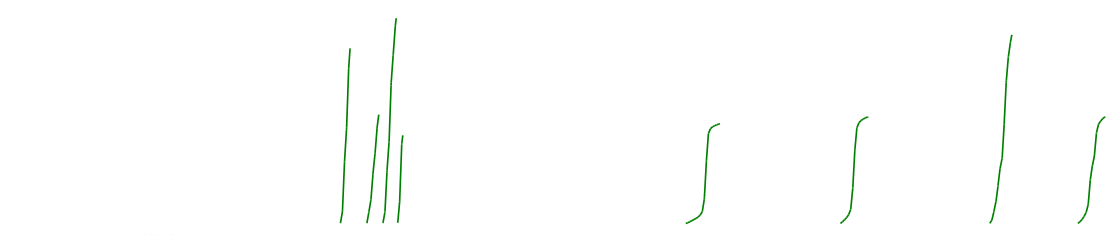

궁ㅇㅇㅇ

i

14000

$\rightarrow 1$

13000

$-12000$

$-11000$

10000

9000

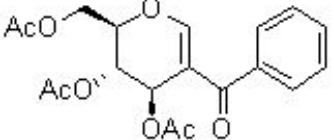

$3 a$

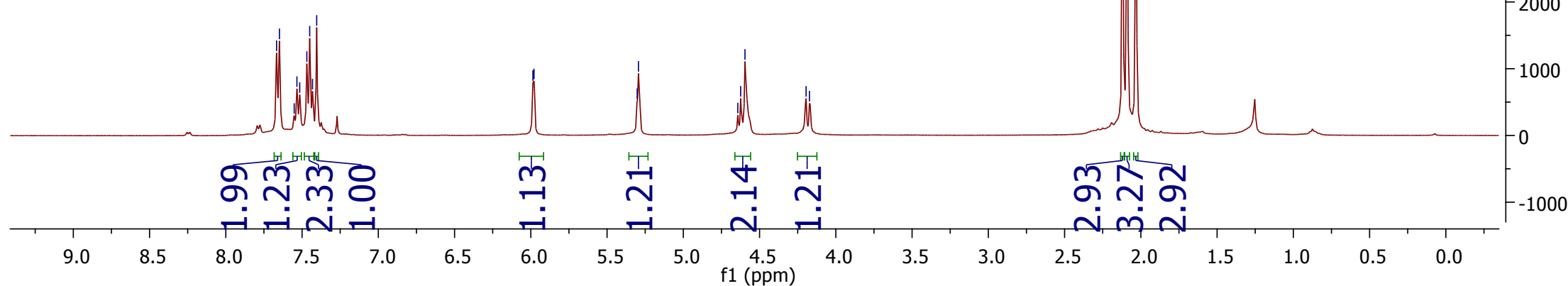




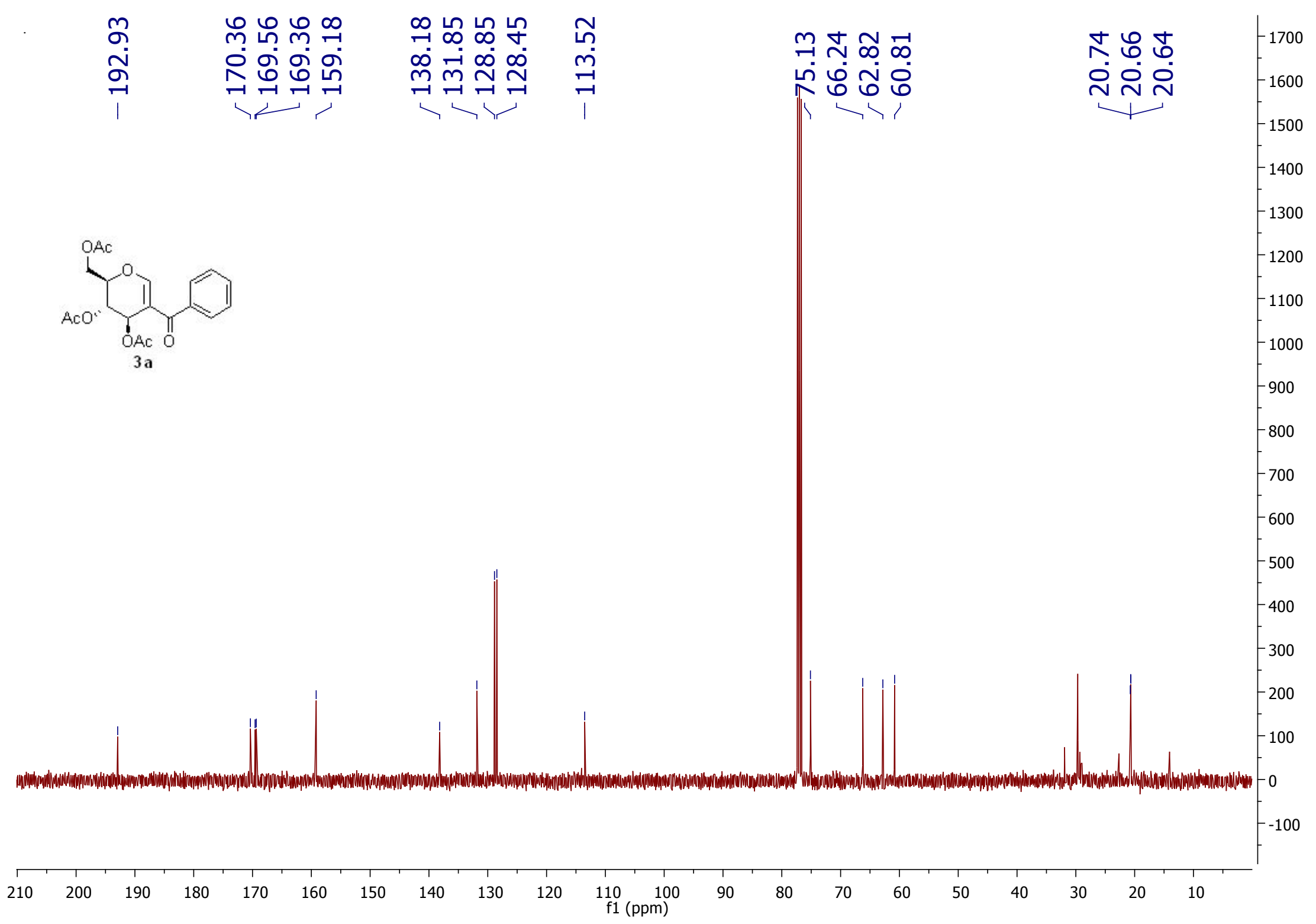




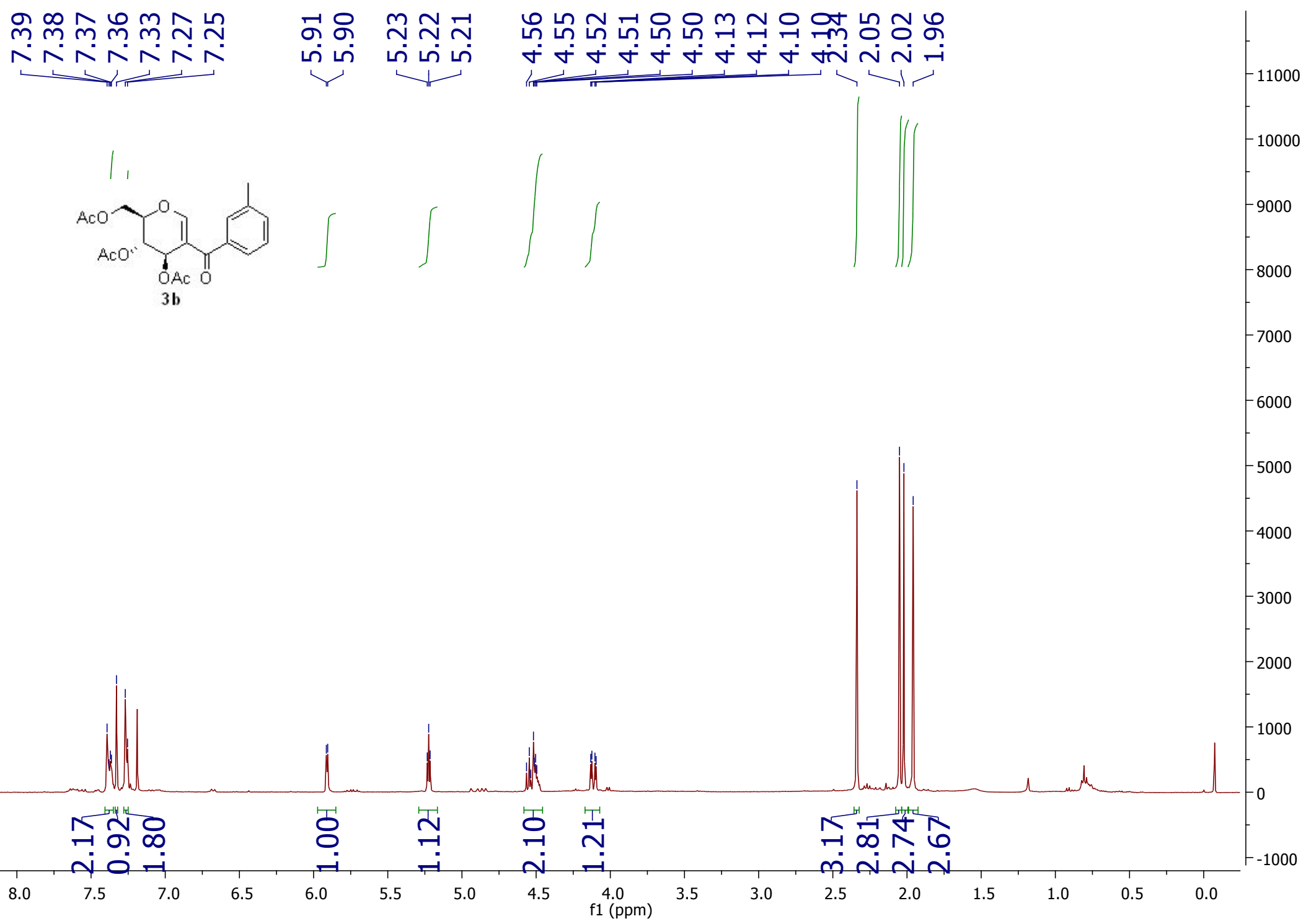




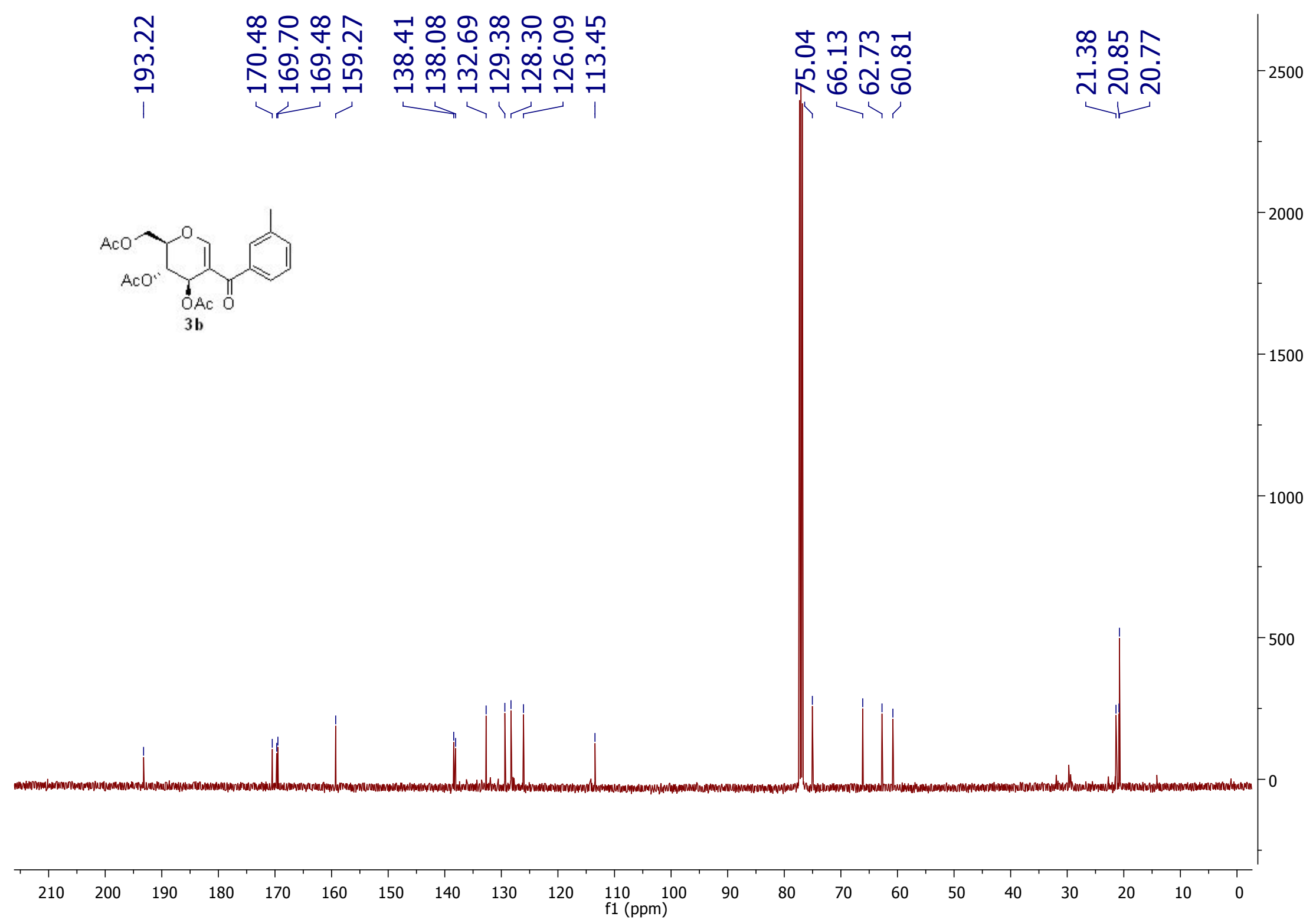




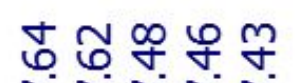

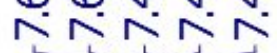

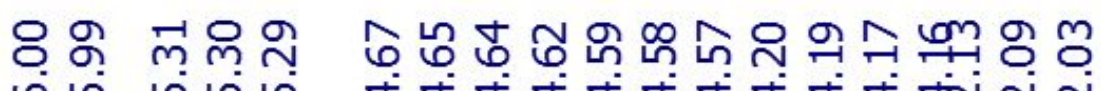

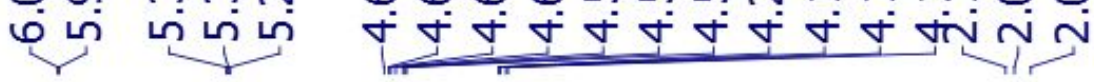

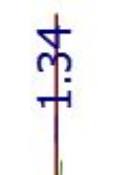

$-15000$

$\Rightarrow 1$

$1 / 1$<smiles>COC(=O)OC1C(C(=O)c2ccc(C(C)(C)C)cc2)=COC(CO)C1O</smiles>

$\int \rho$

$3 \mathrm{c}$

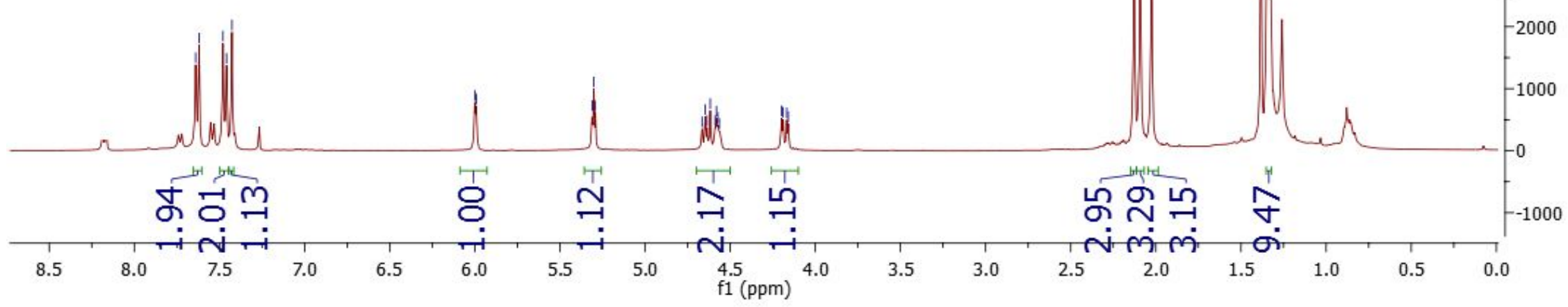




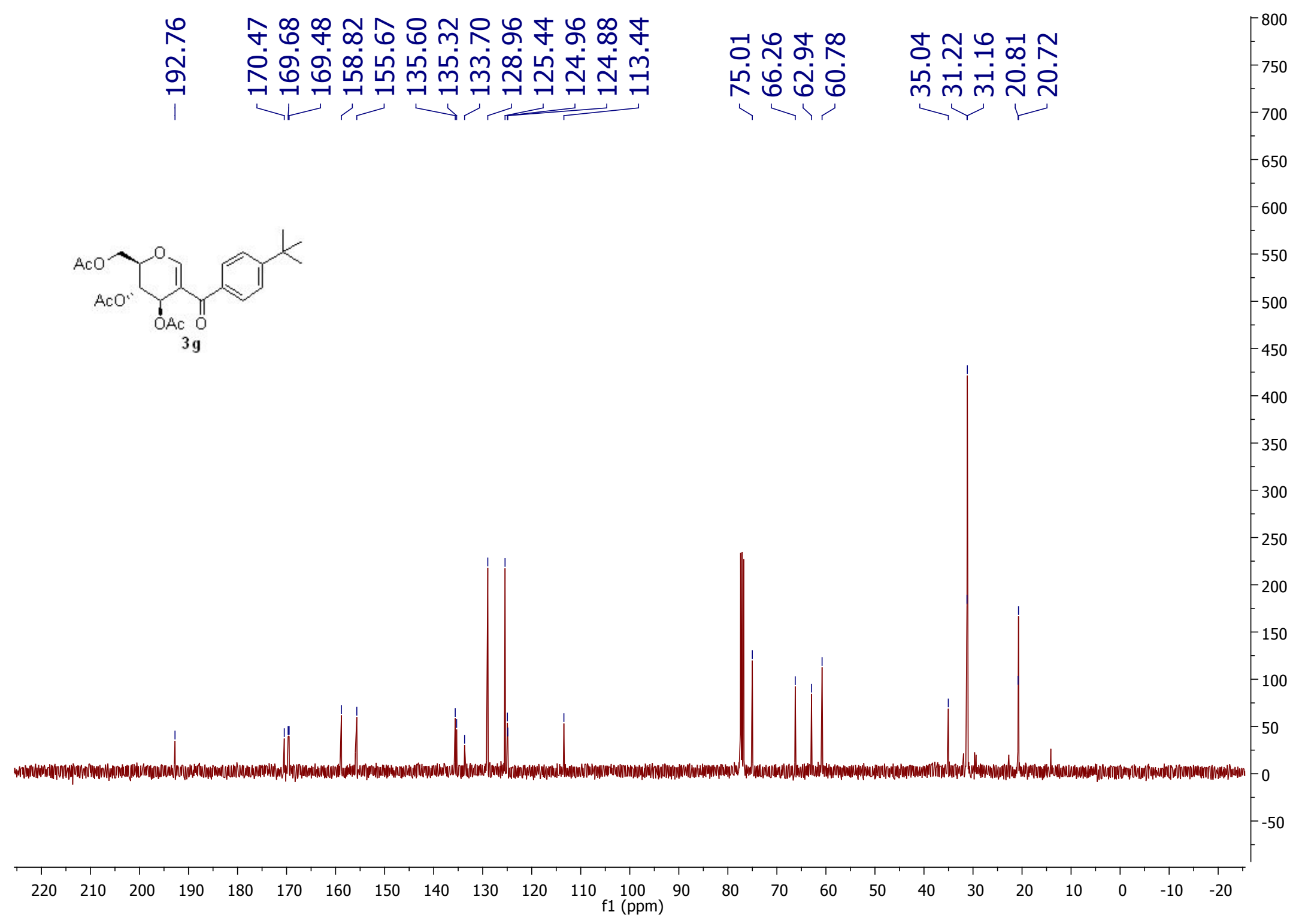




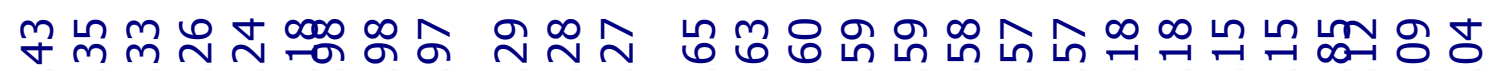
ヘNヘNヘ
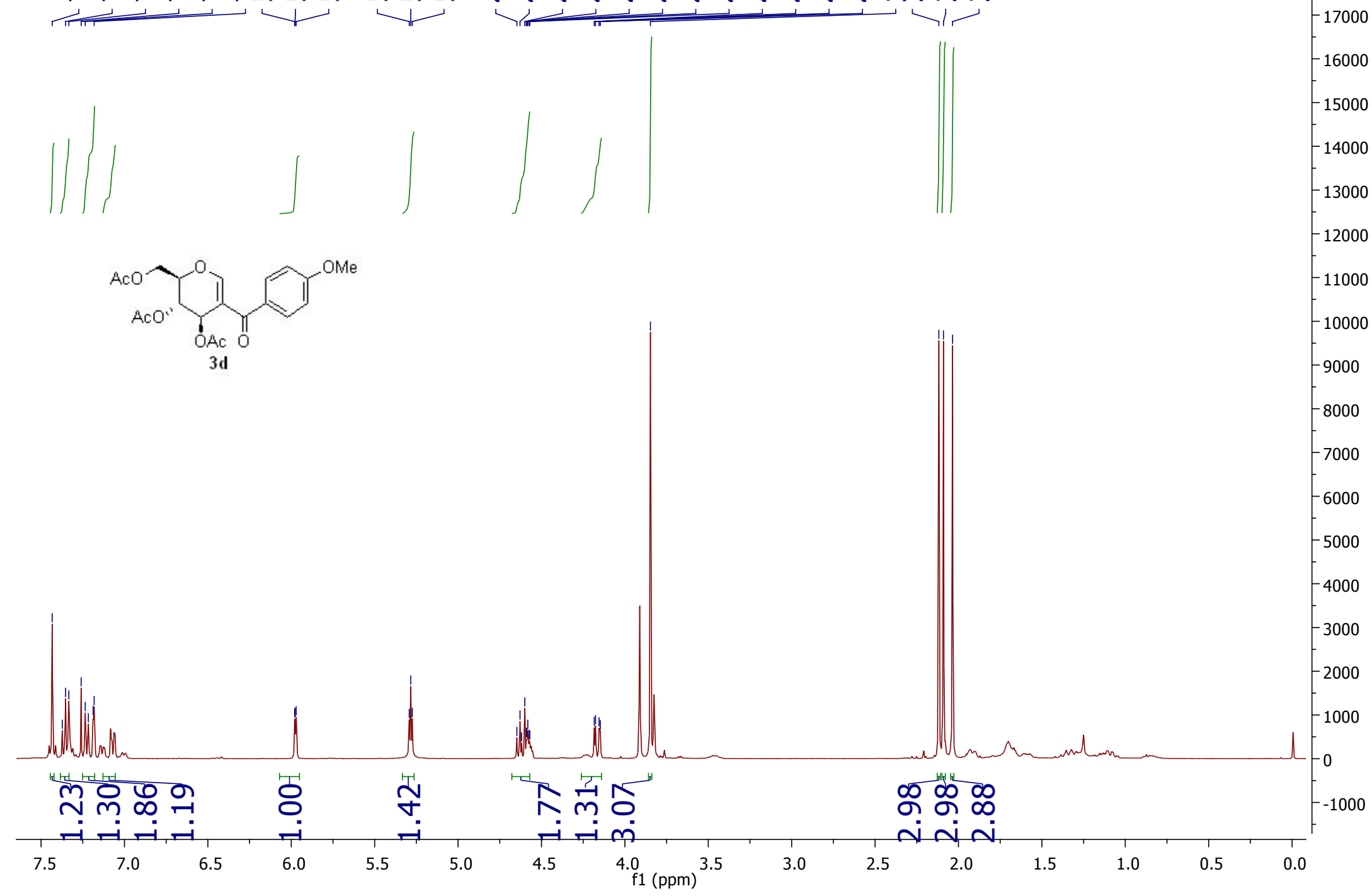







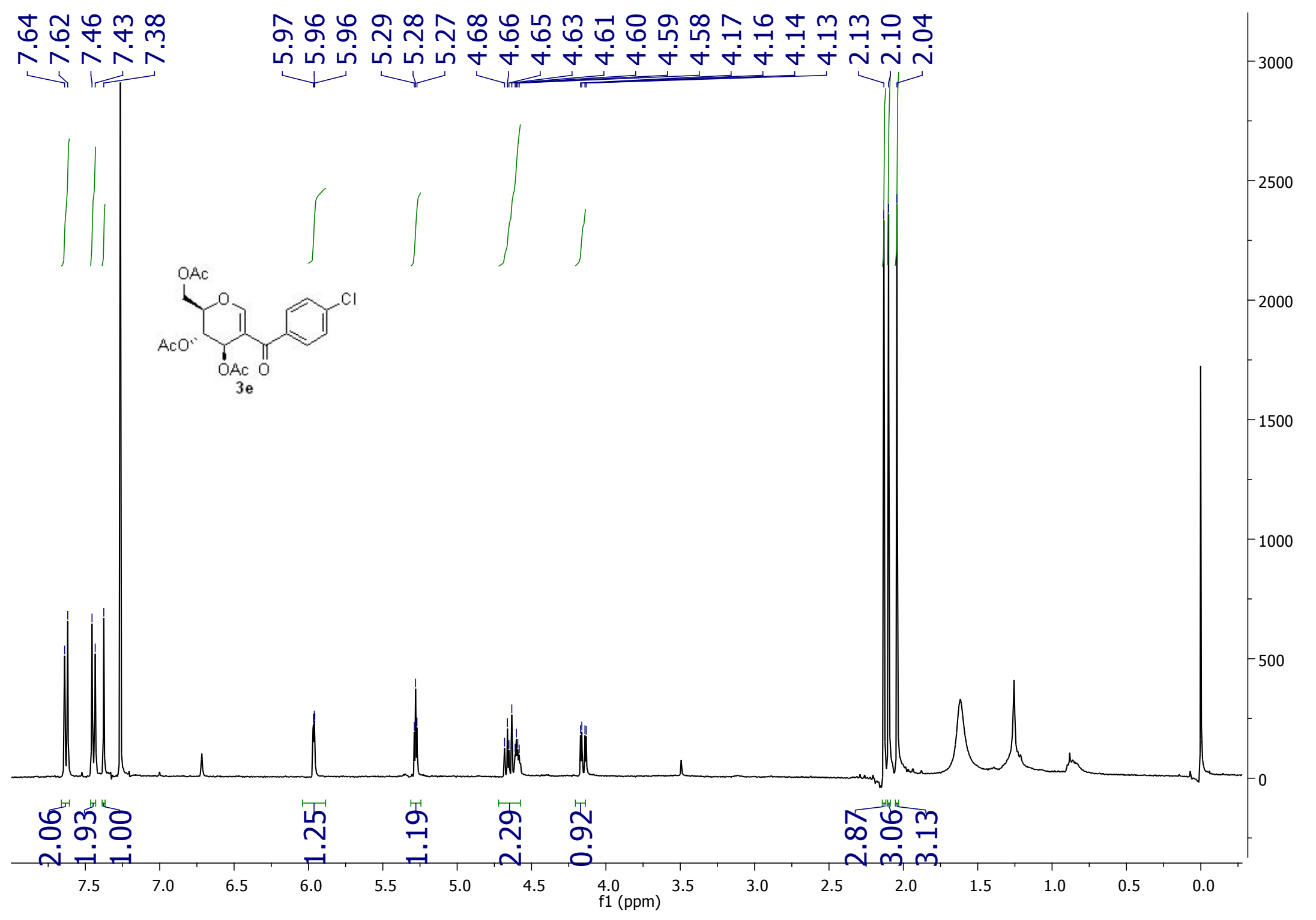




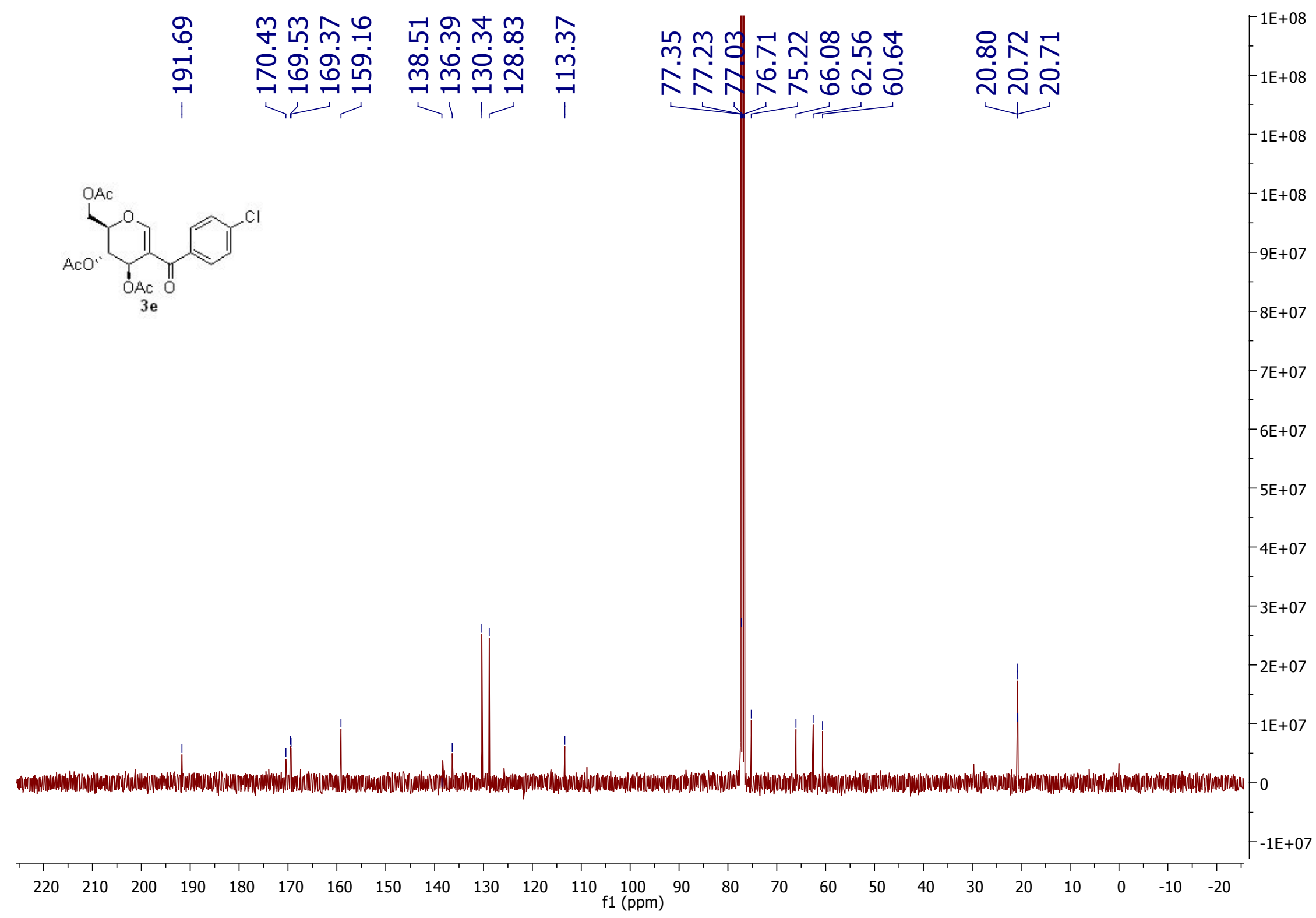




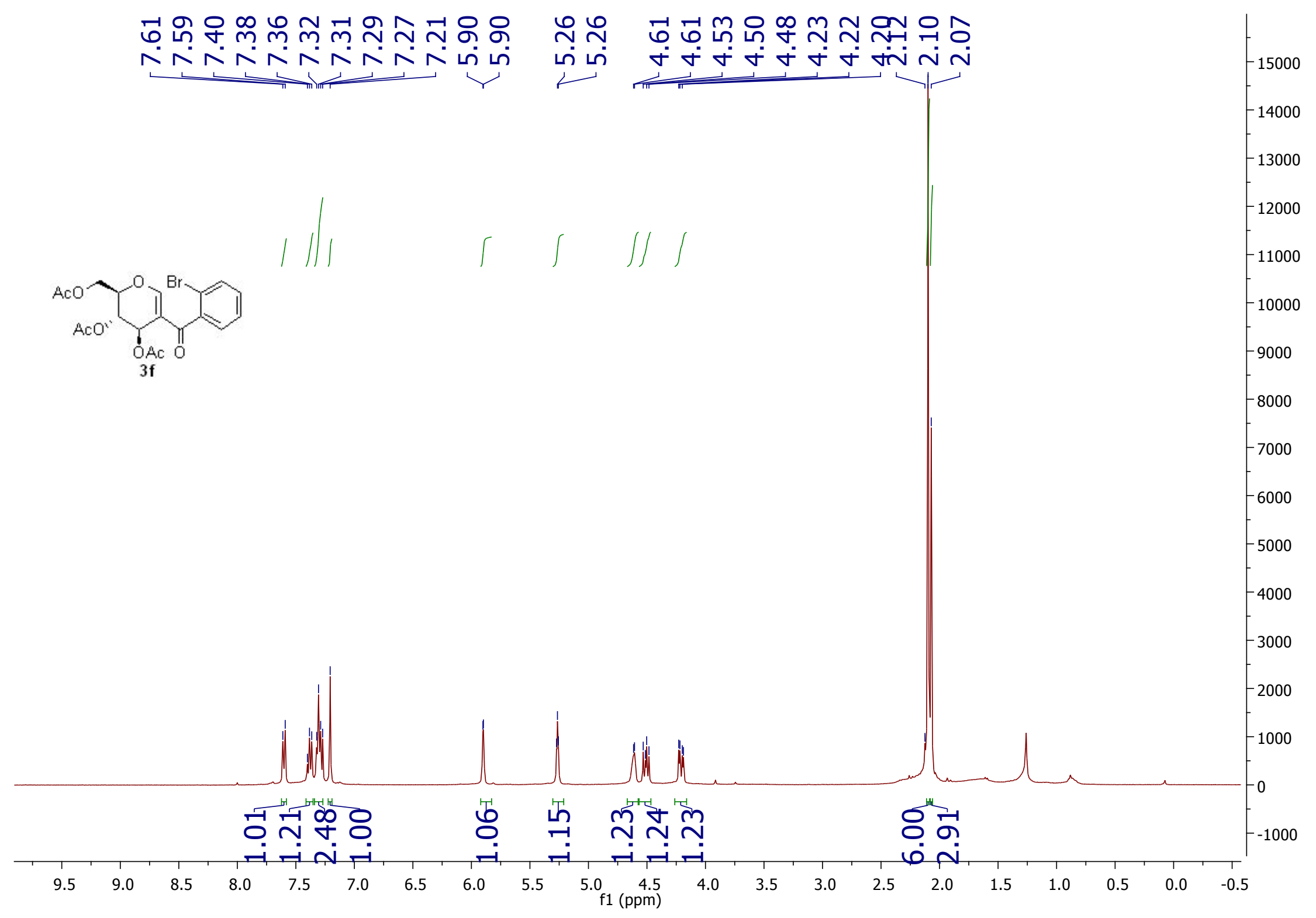




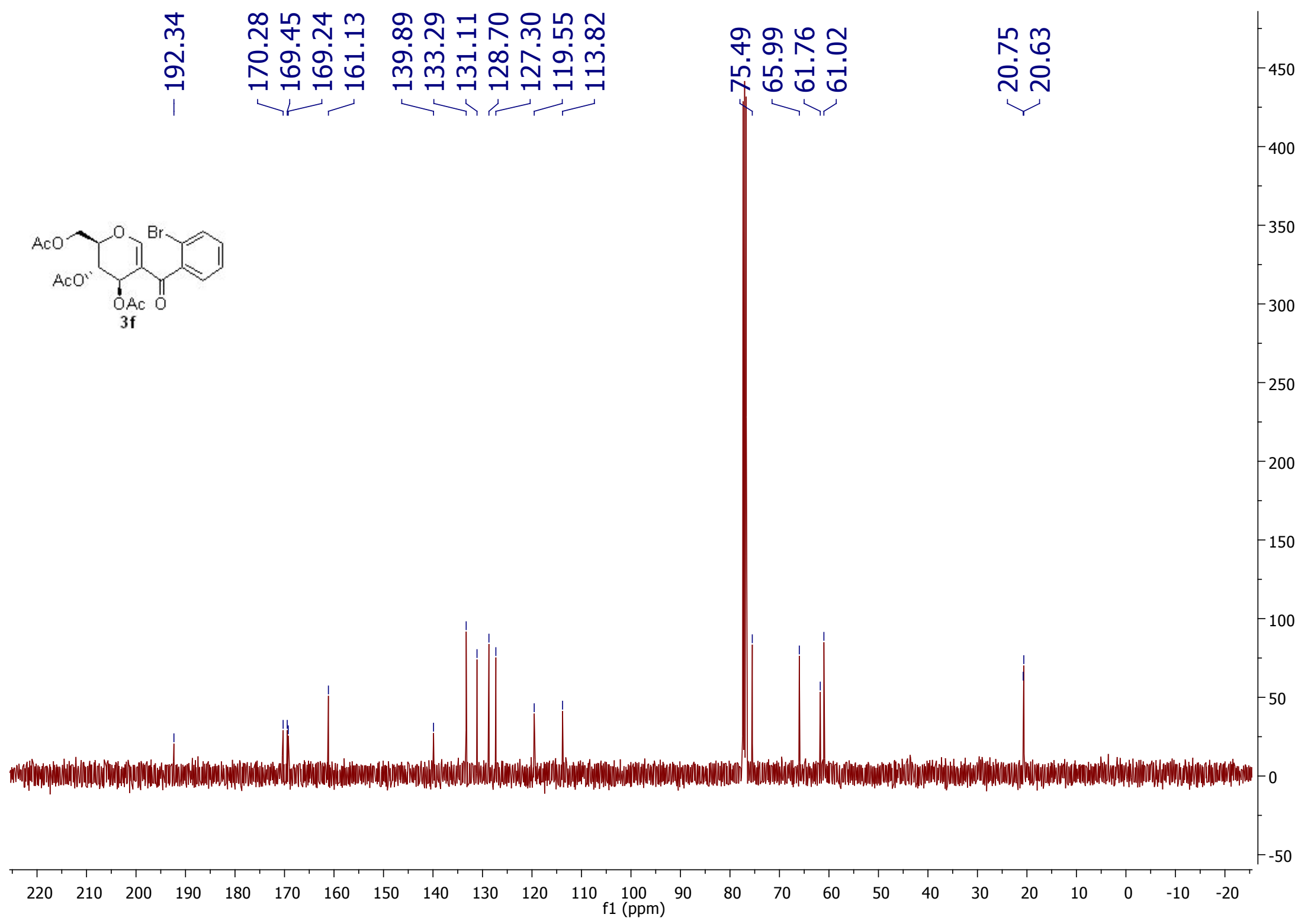




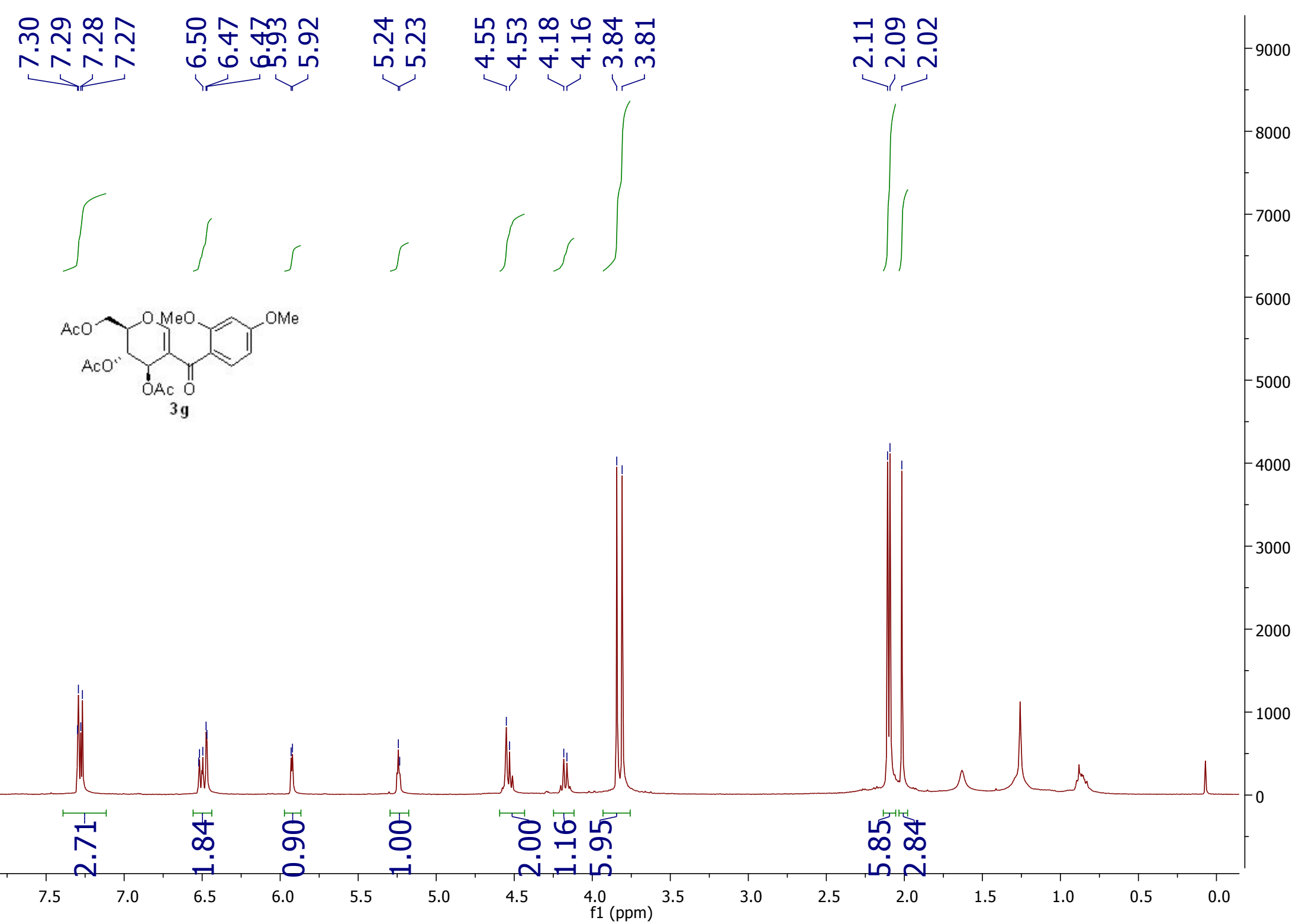




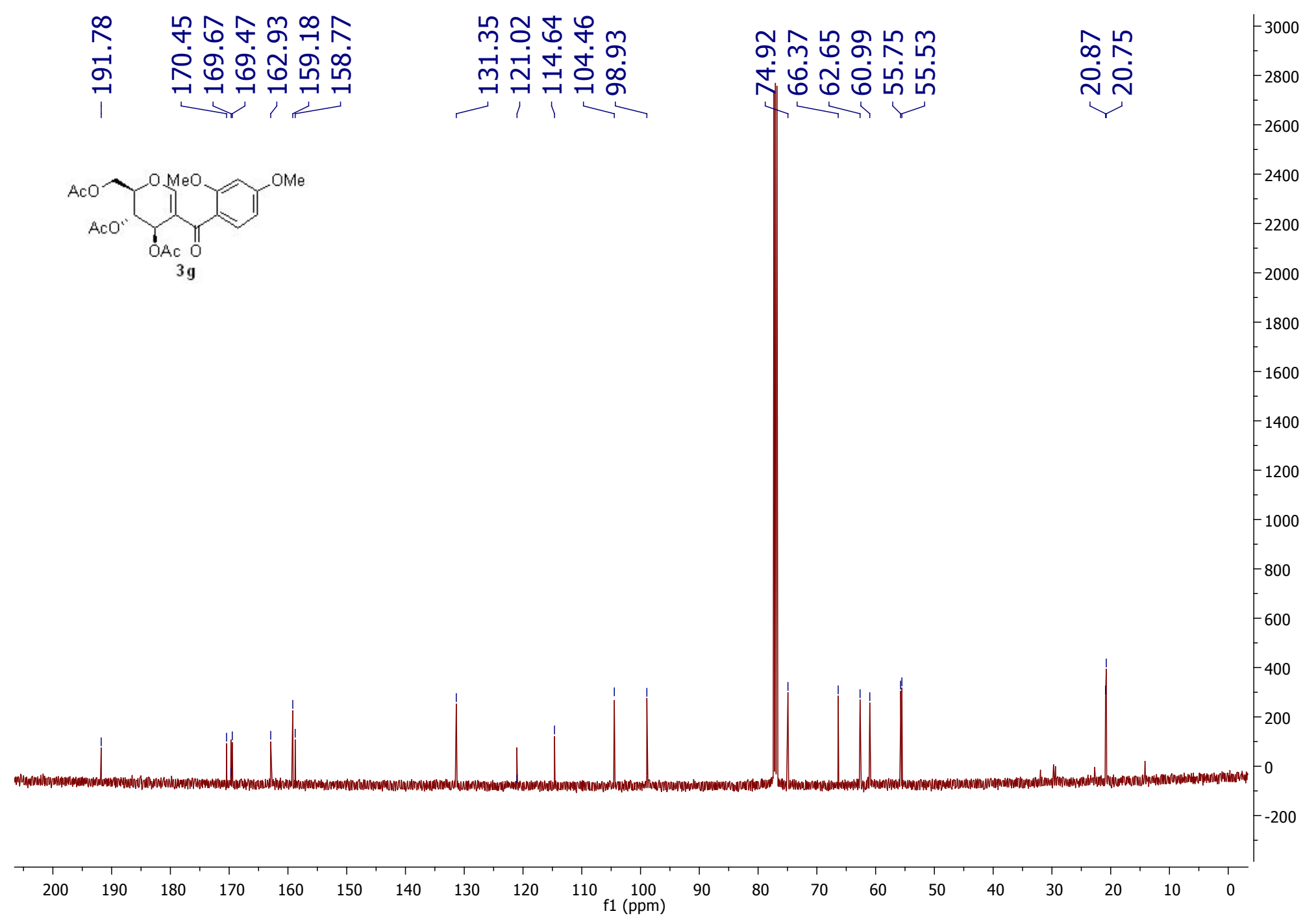




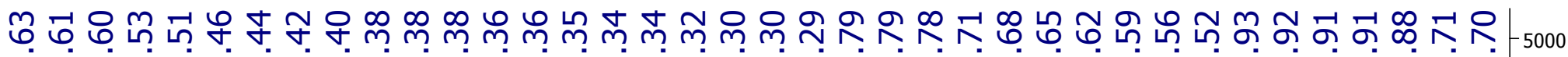

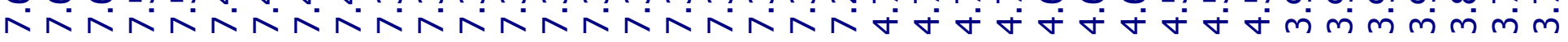

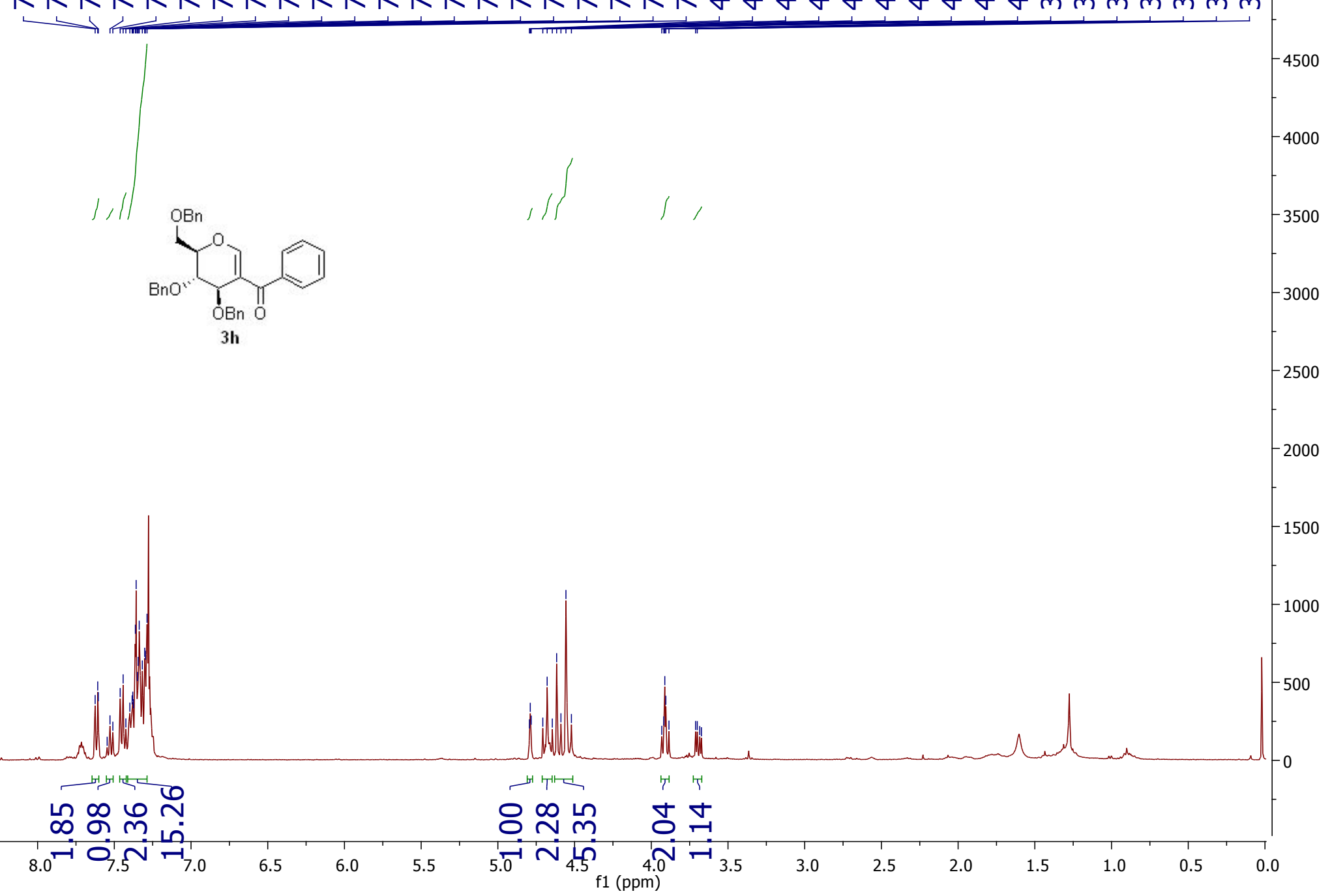


ติ

กั่

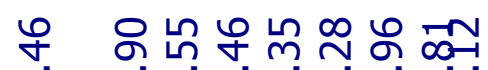

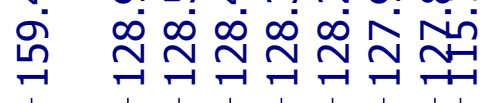

$\sum_{\substack{\mathrm{OBn} O \\ \mathbf{3 h}}}^{\mathrm{Bn}}$
ఫ

रำत

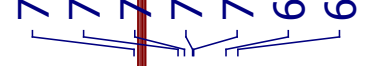

1800 


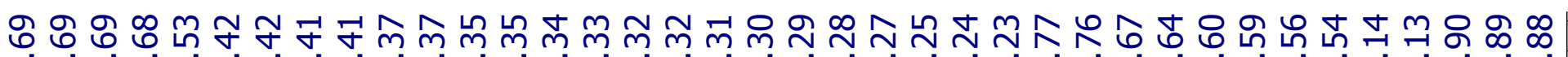

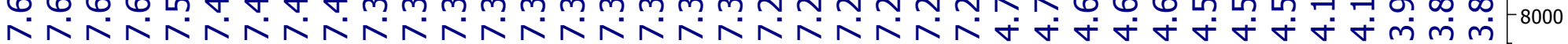

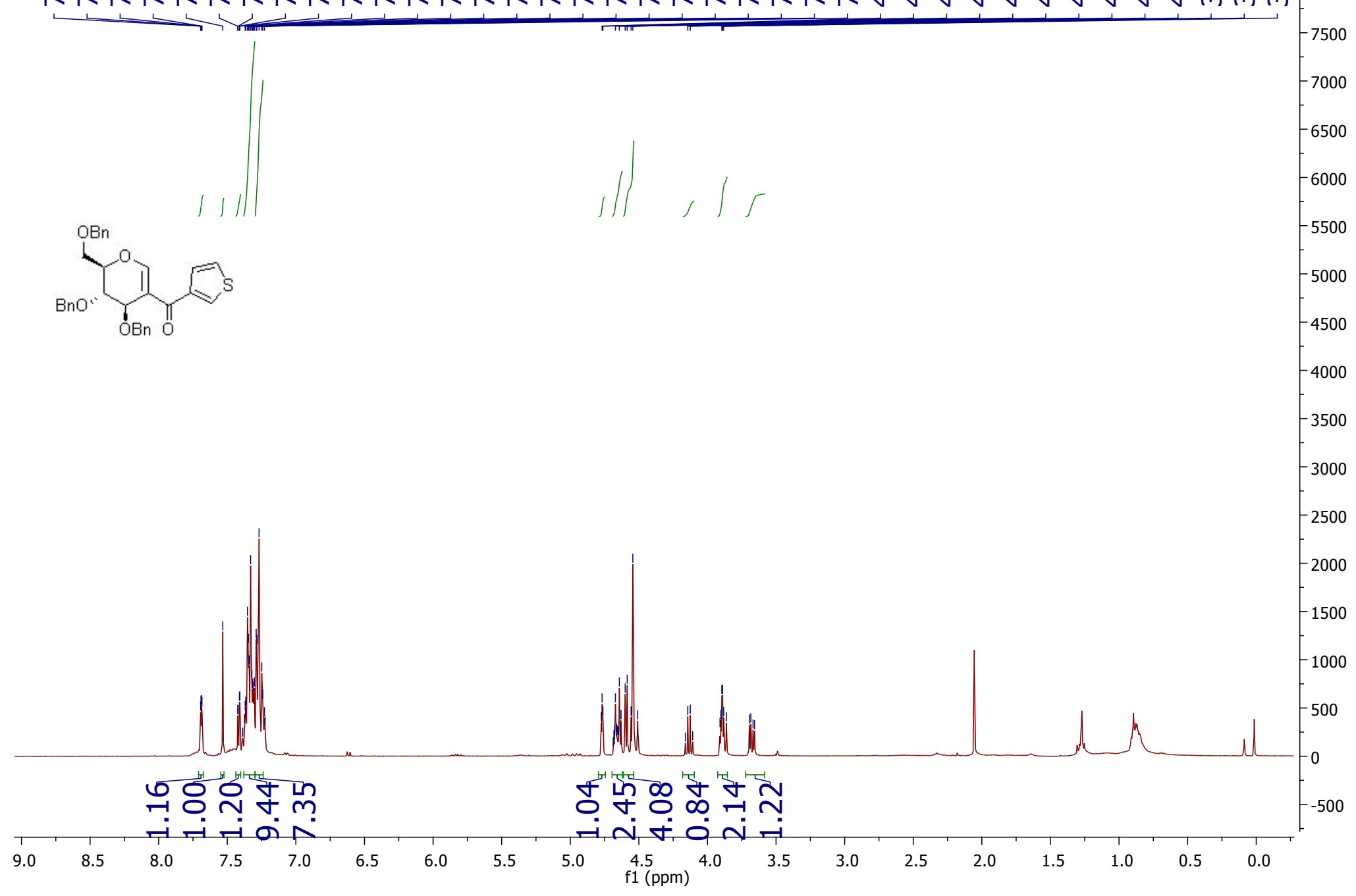




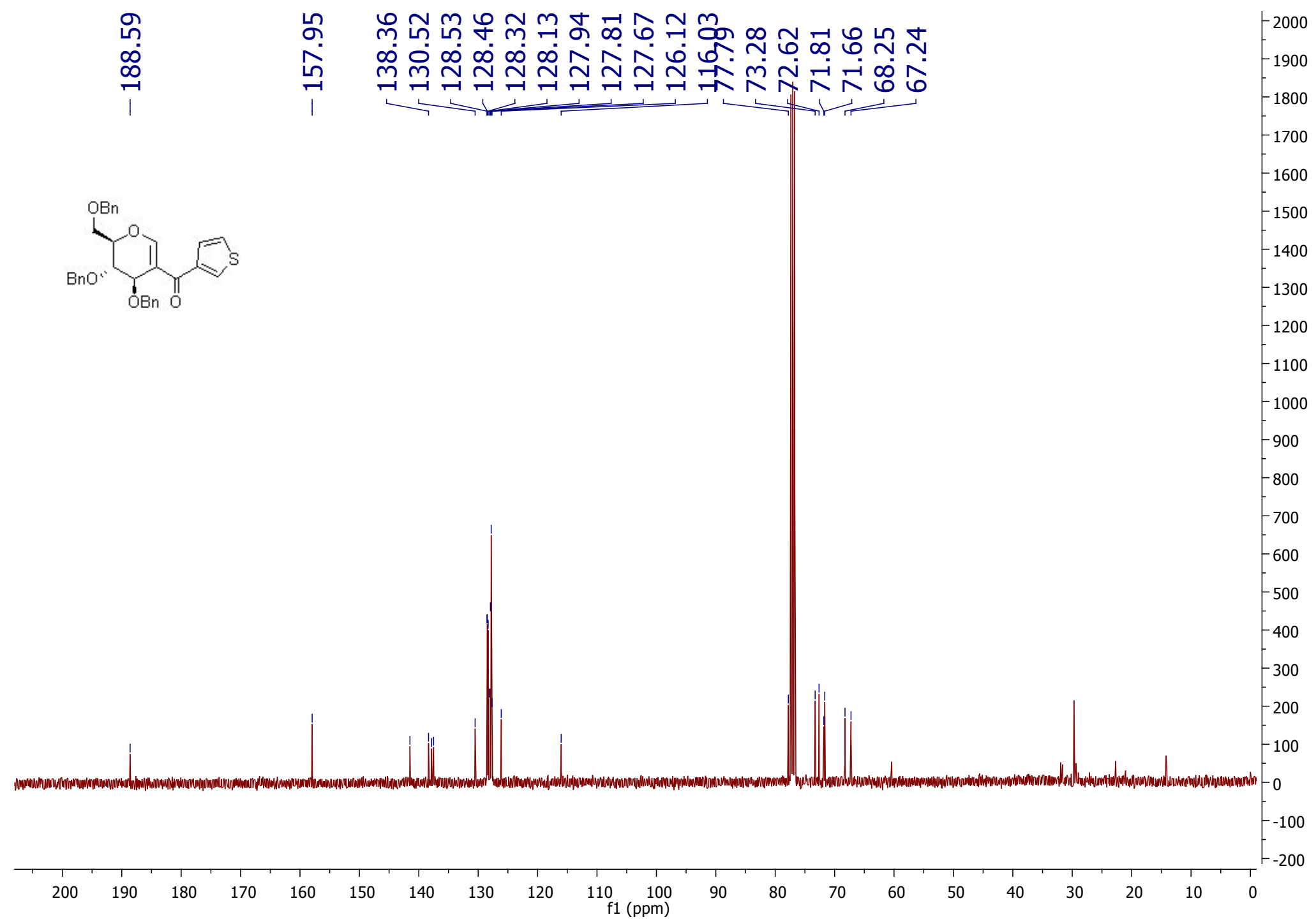


ம

NヘNヘNヘ



ऍ
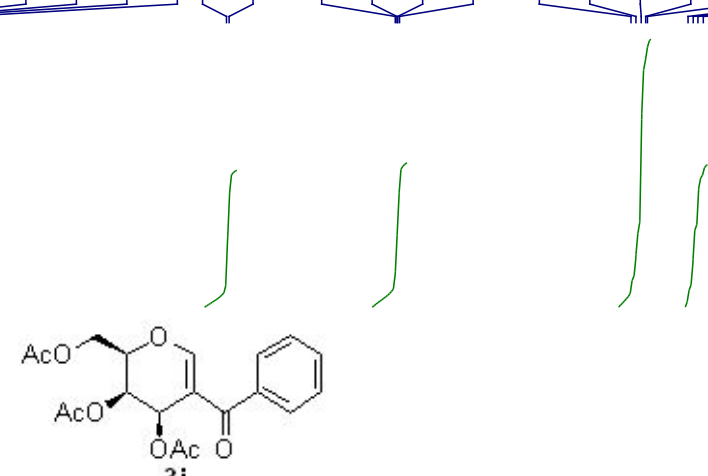

$3 \mathbf{j}$

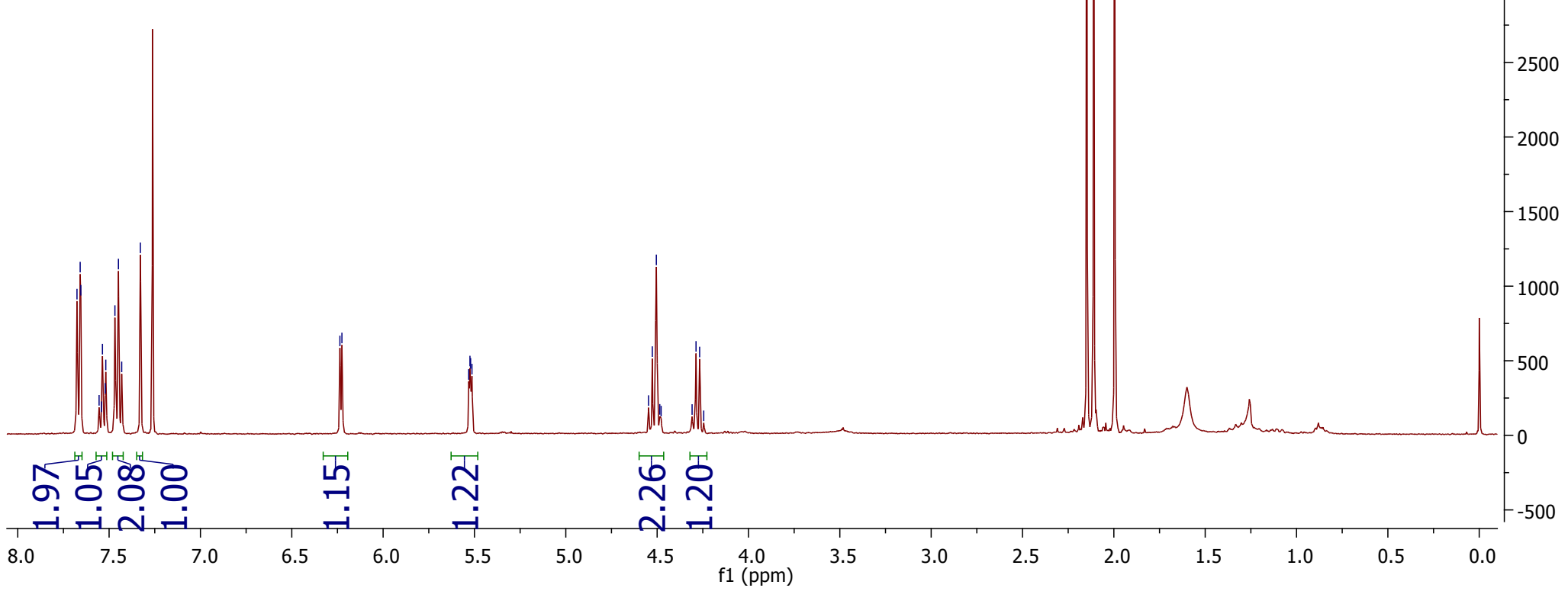




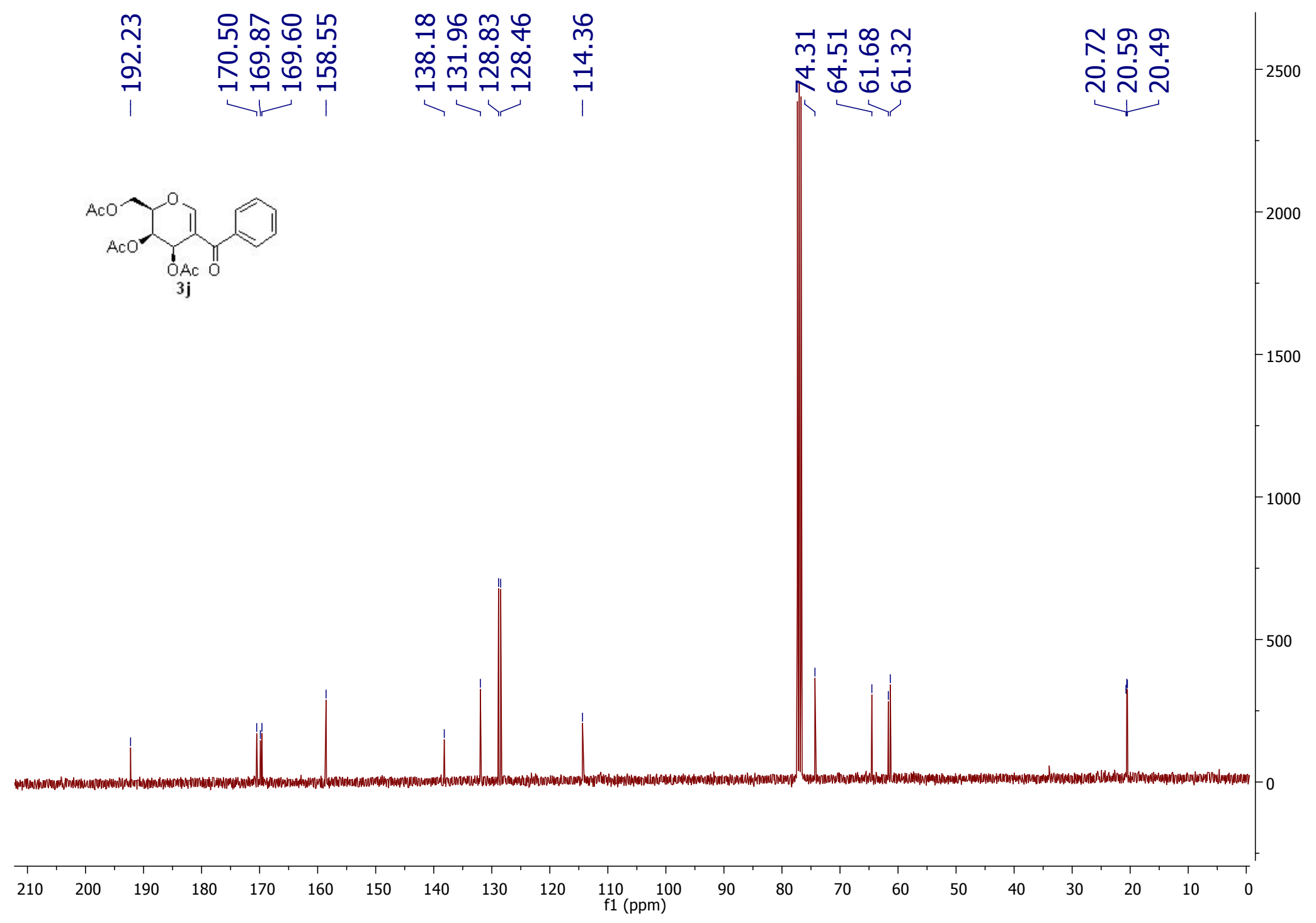






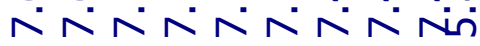

$1 /$<smiles>CC(=O)OC1C(C(=O)c2ccccc2)=COC[C@H]1C(C)=O</smiles>

$3 \mathbf{k}$
IIIII,

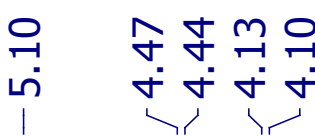
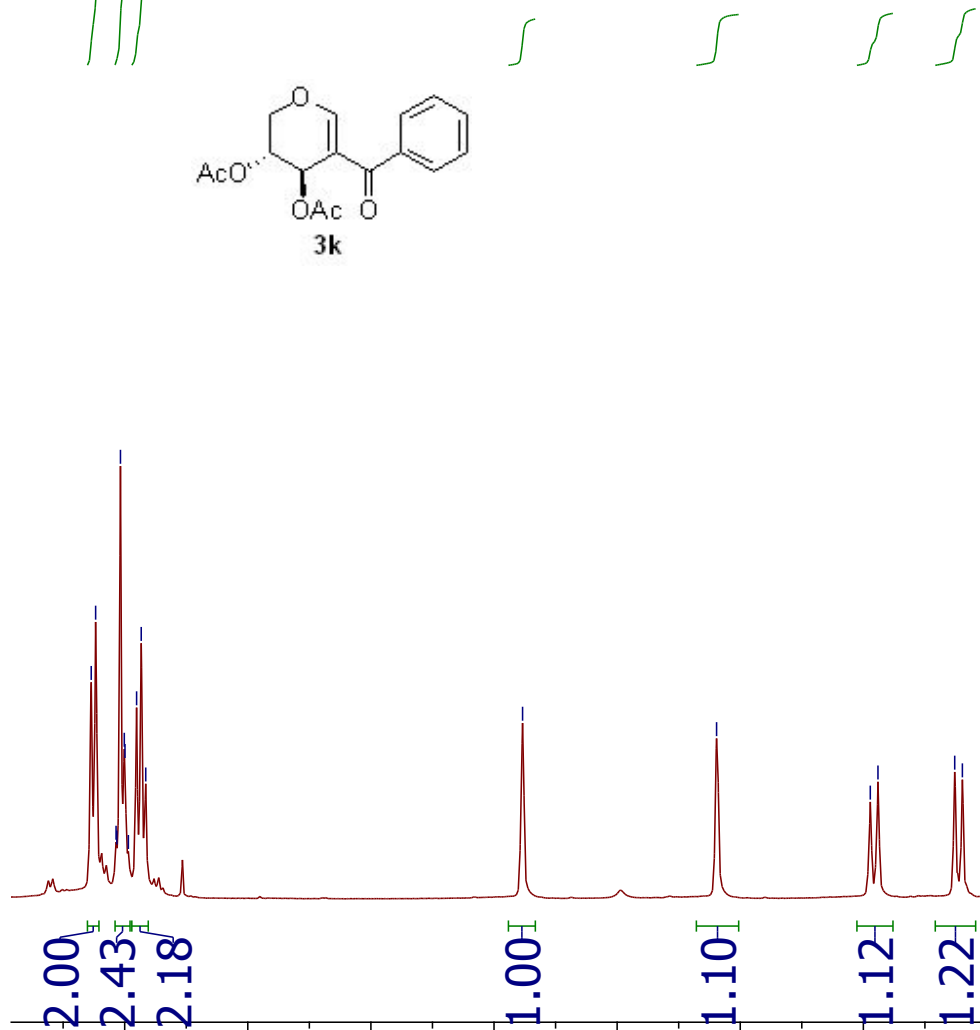

7.5

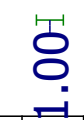

6.0

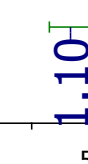

\&용

in

4

9000

8000 


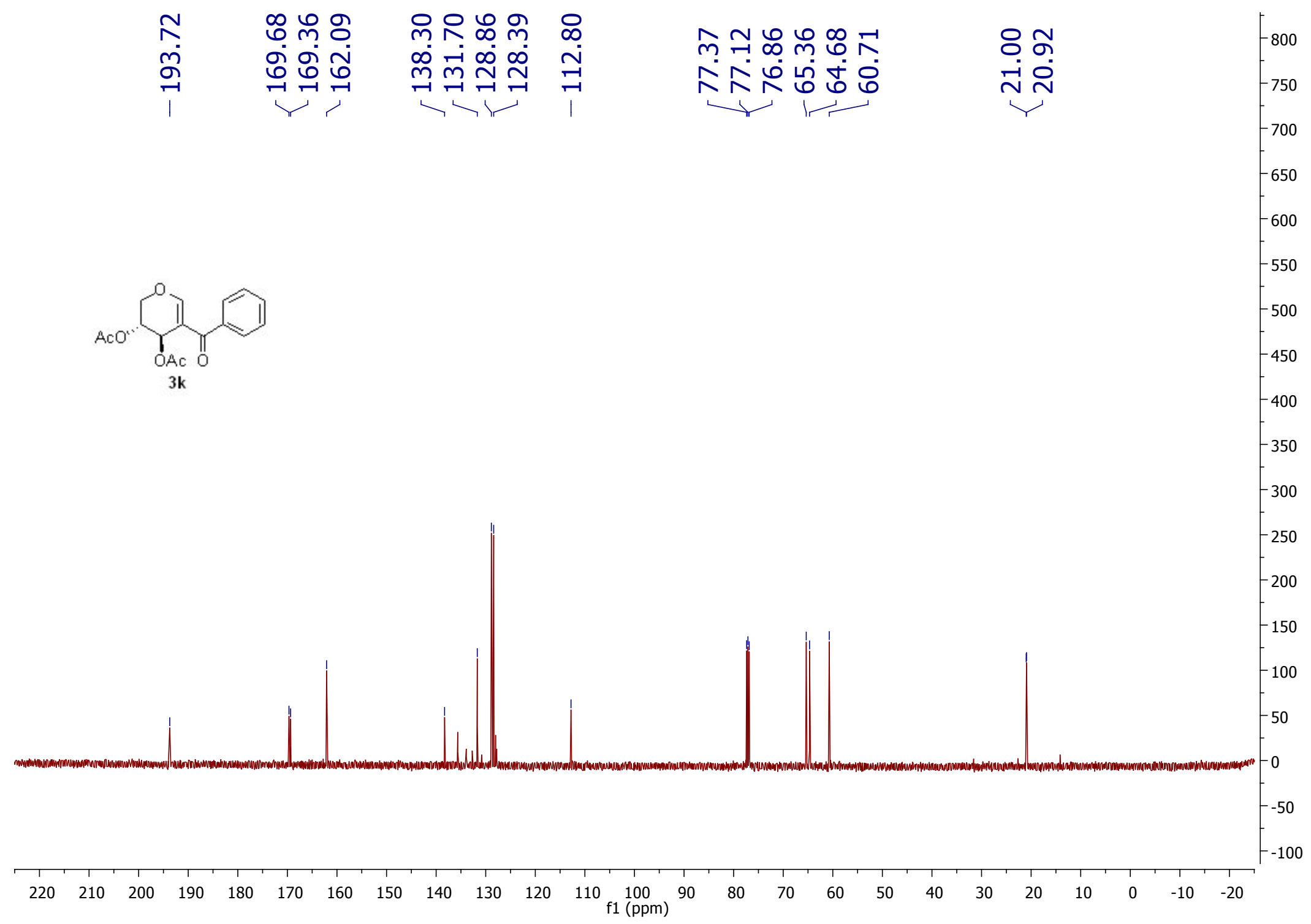




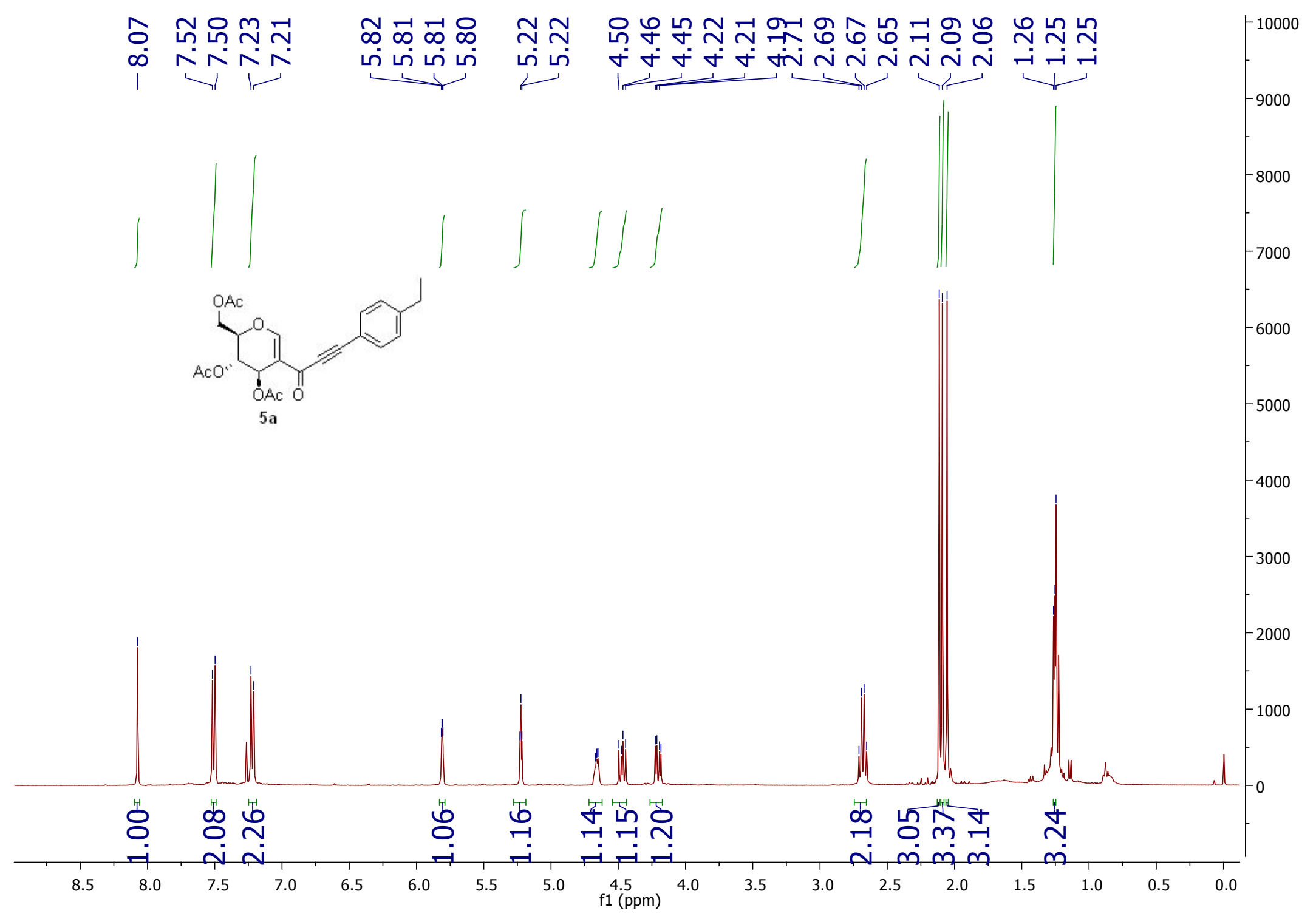




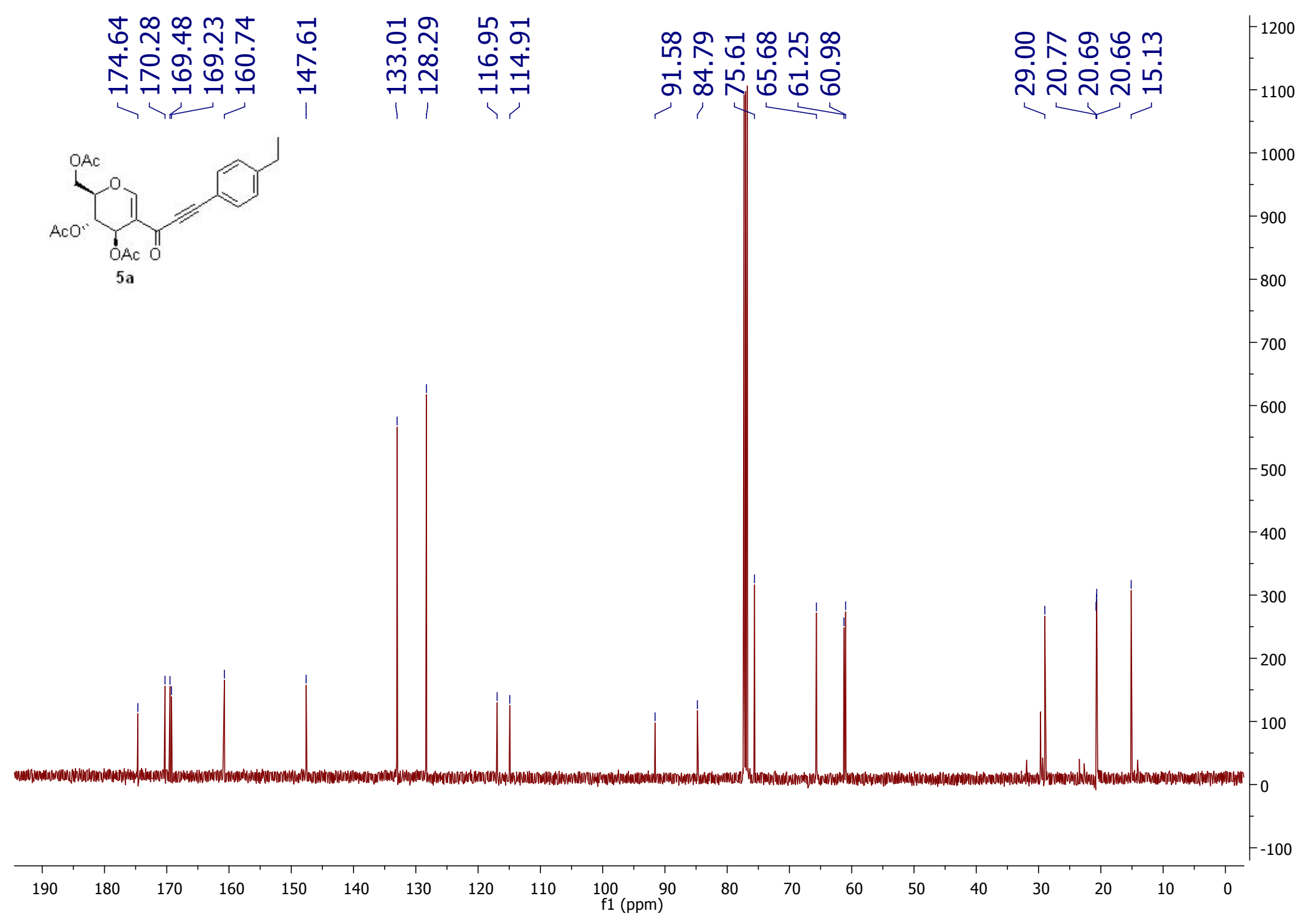


$\infty$ ำ ำกำกำ

$\infty N-N$

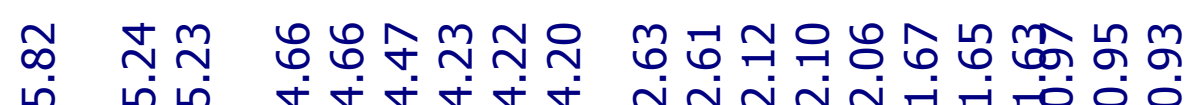

5500

$1 \longrightarrow$

r
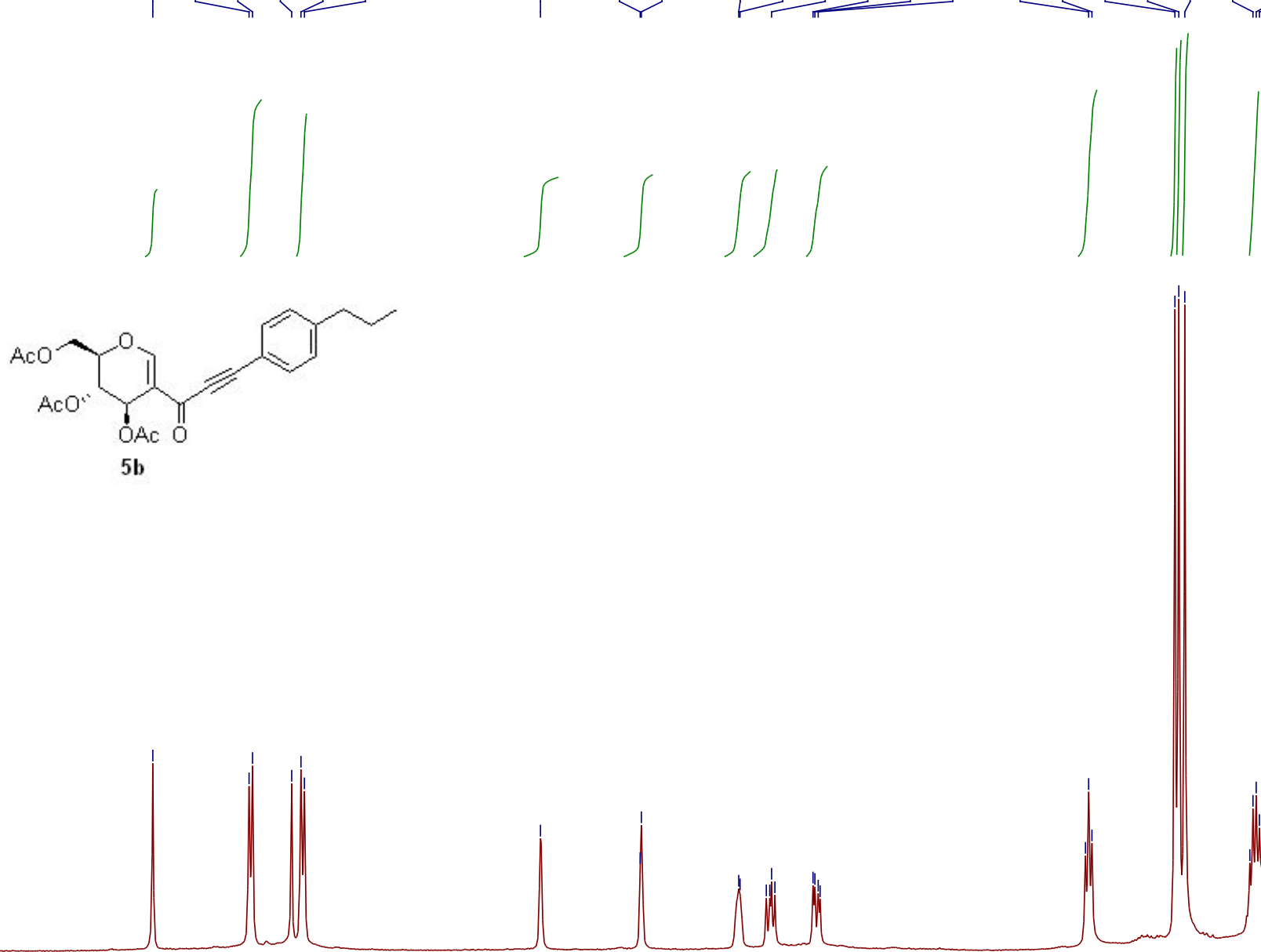

3500

$-3000$

$-2500$

2000

1500

1000








○ீ नં்

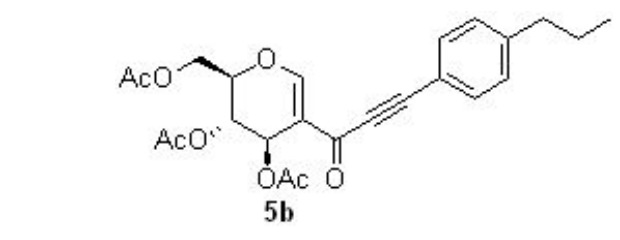

욱 뉘용ㅁㅇㅛ

m ㅎํ윰ำ

12
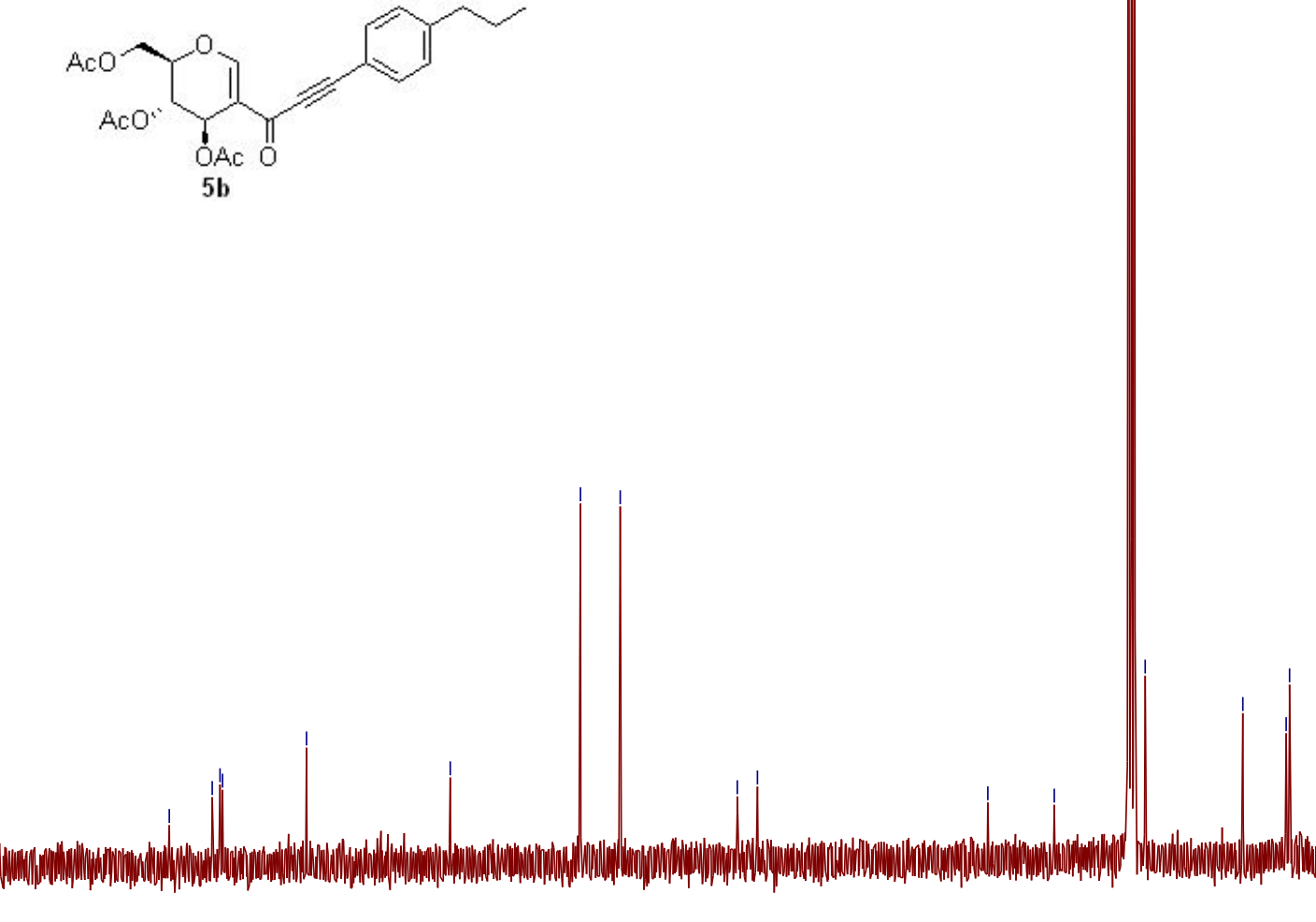


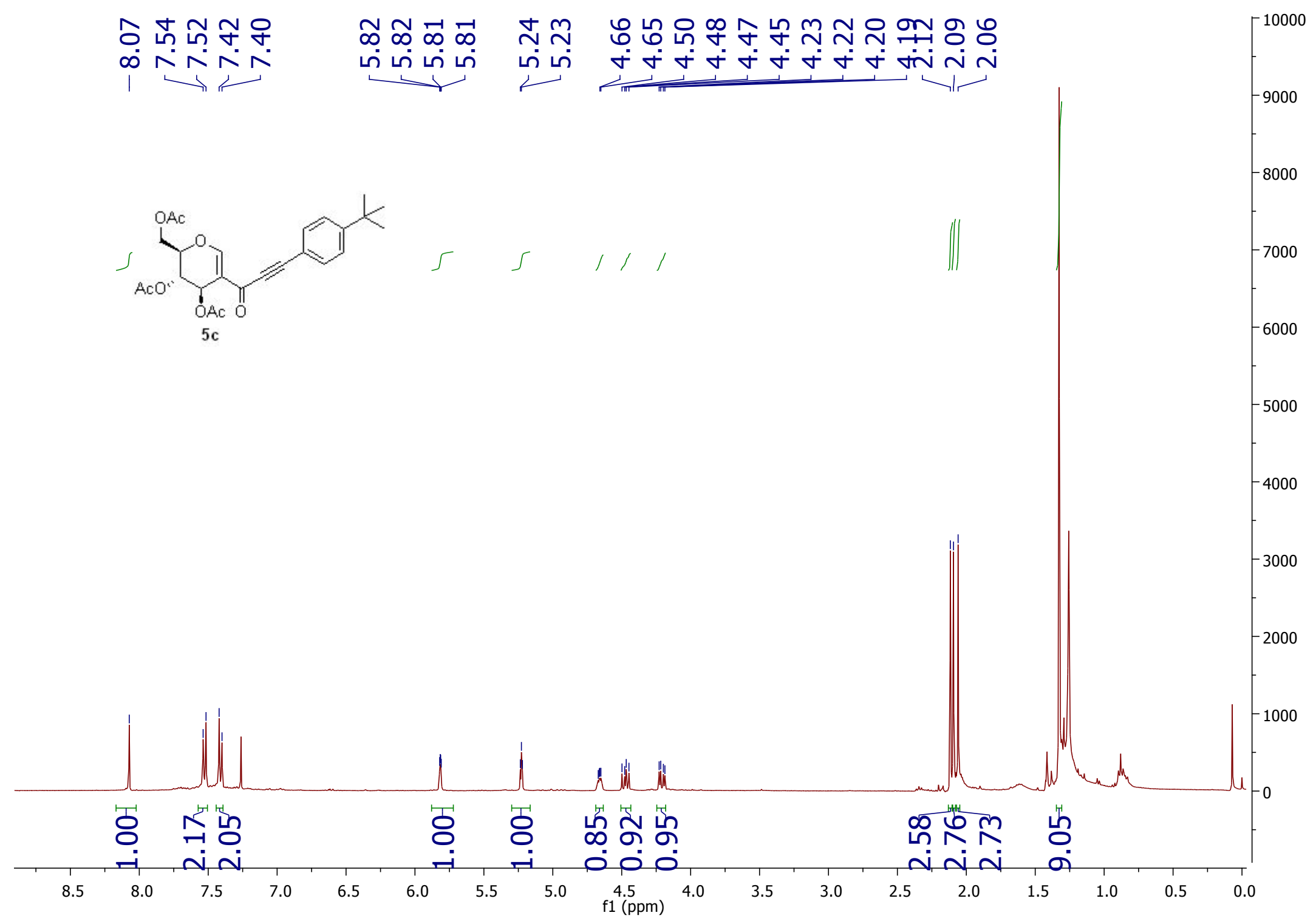




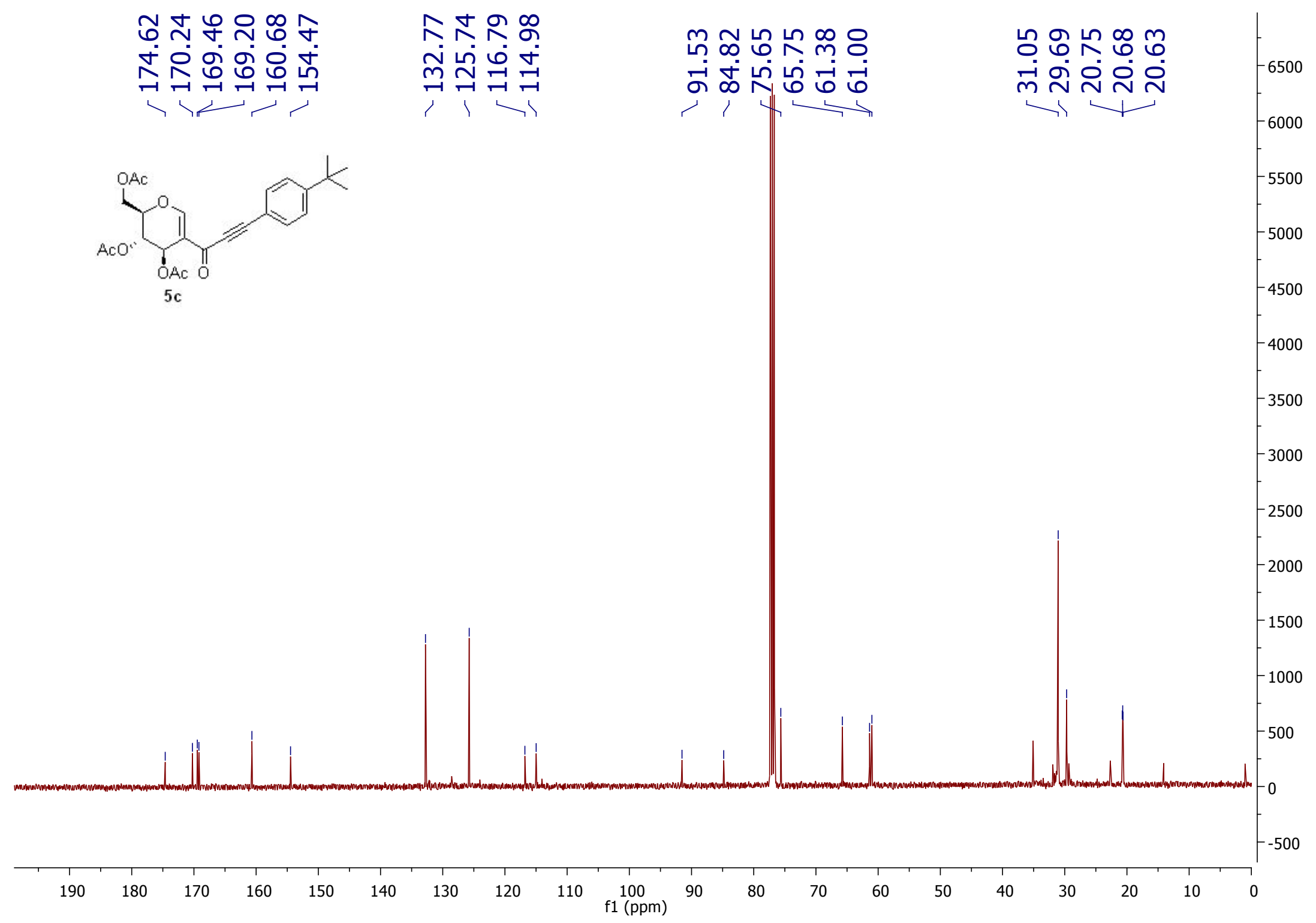




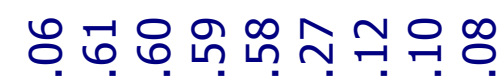

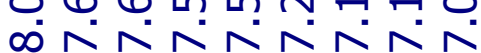

$\infty$ 끄ำ

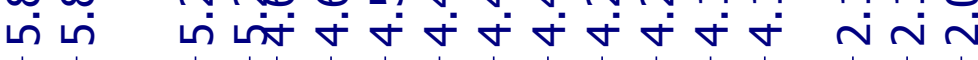

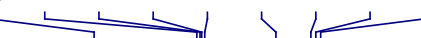

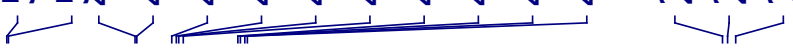
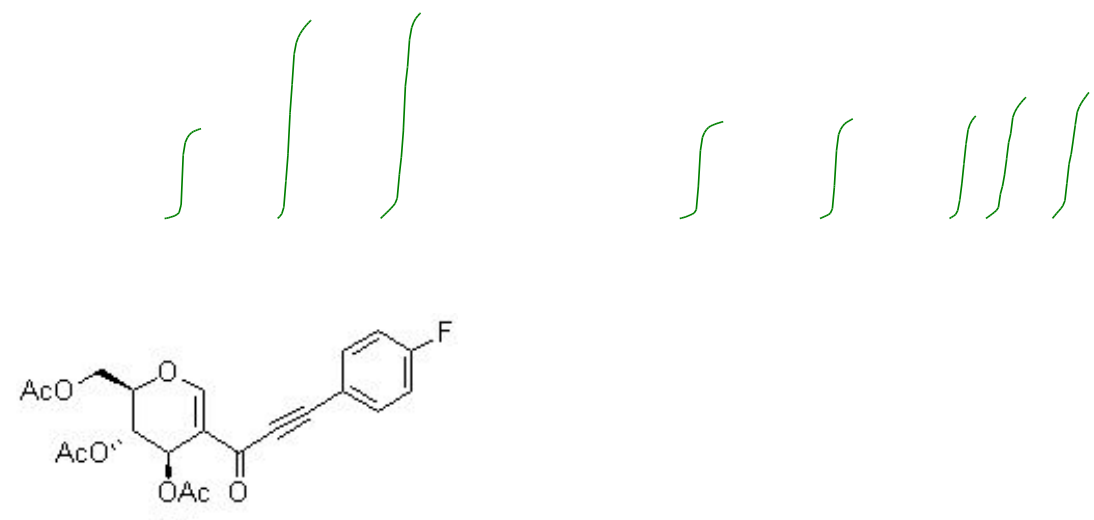

$5 d$

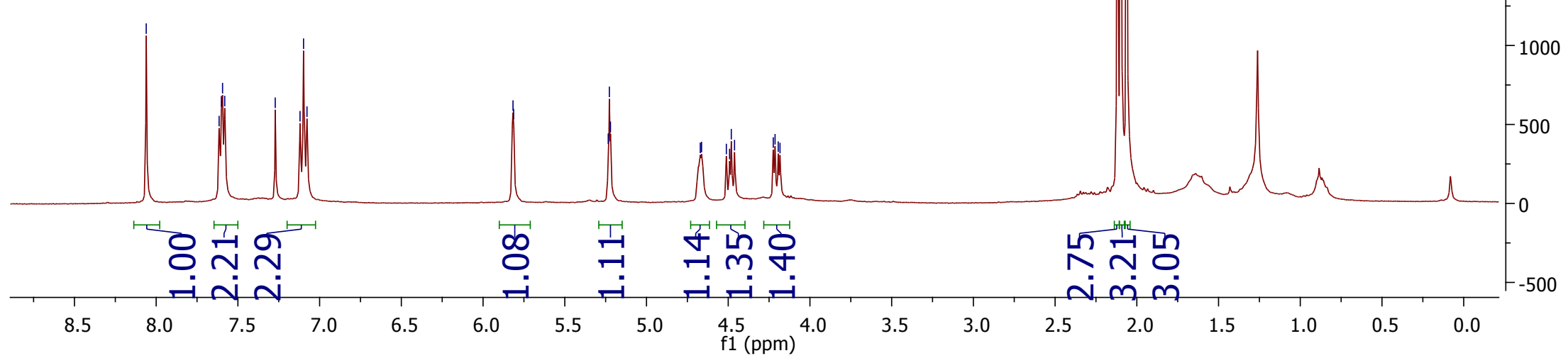


ำฟำำㅇํㅇ

ரீ

न규규귝

윽으

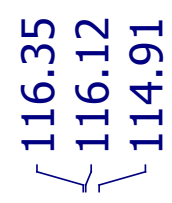

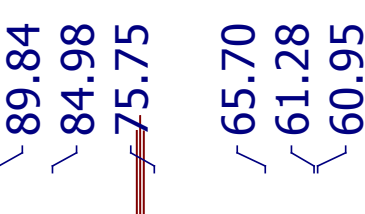

부ำ



융ํํ

$-1100$

$\longrightarrow$

nand


$180 \quad 170 \quad 160$

150

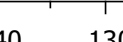

120

110

90
f1 (ppm)

80

70

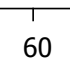

50
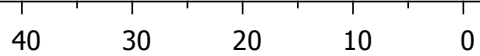



ヘ

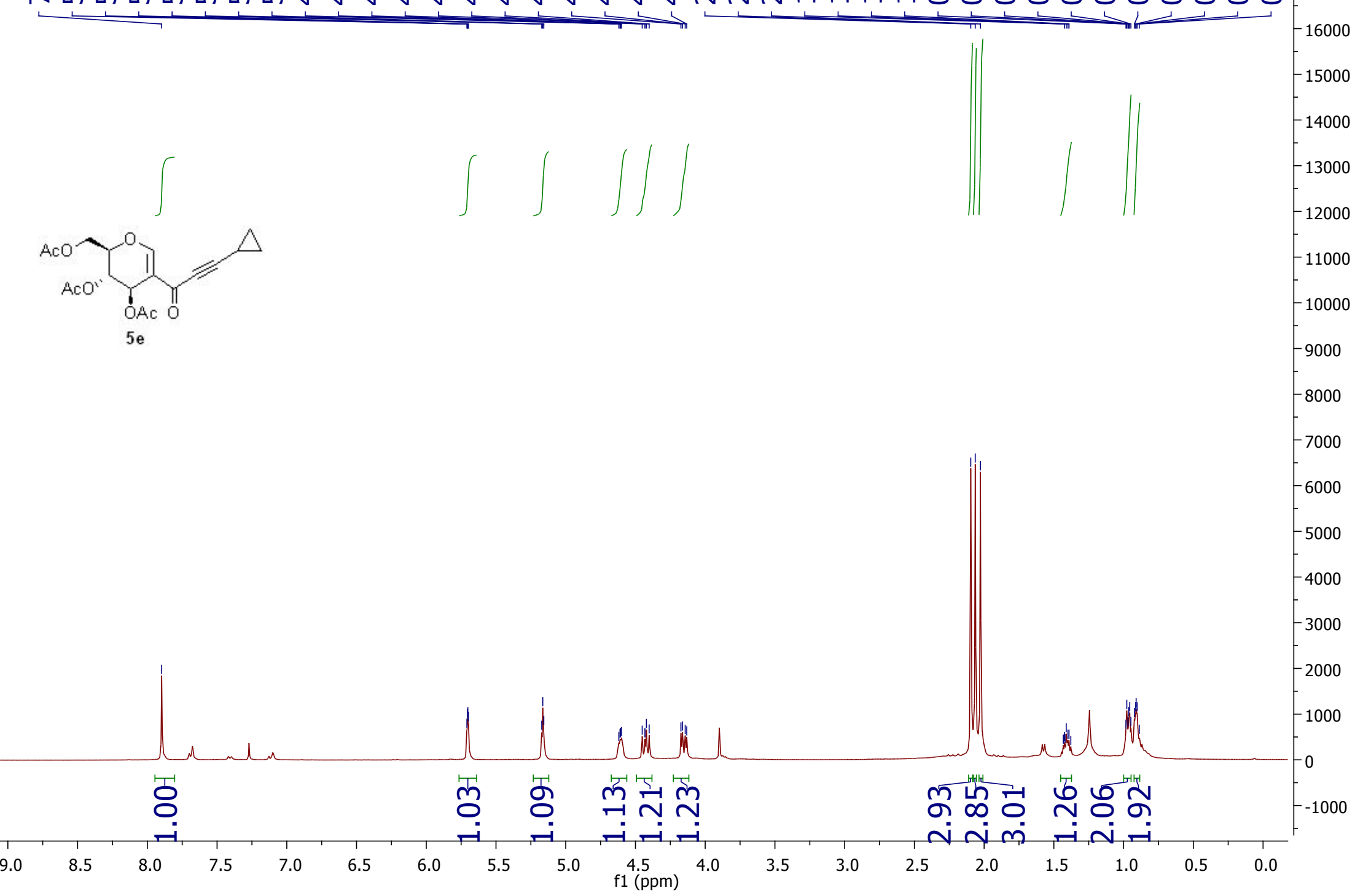


느ำㅇำ

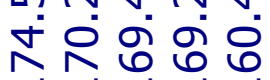

가규 가

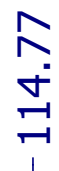

๑

क N

กู

$\checkmark$

9000

$-8000$

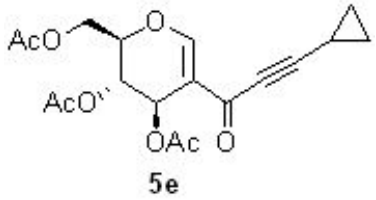

6000

$-5000$

4000

$-3000$

2000

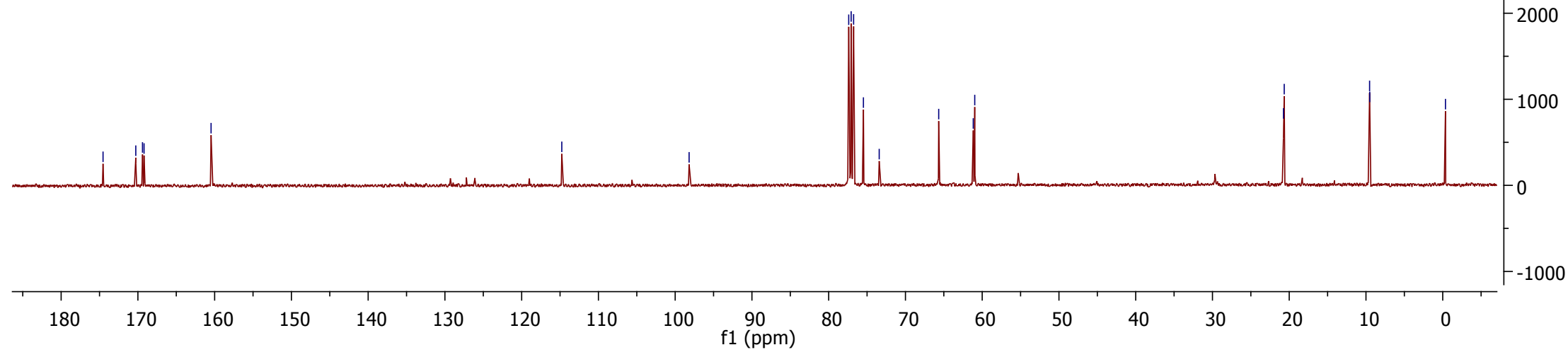


그유 ㅇํำ

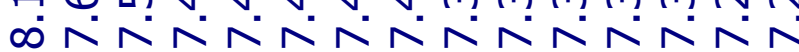

ำ

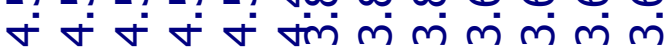

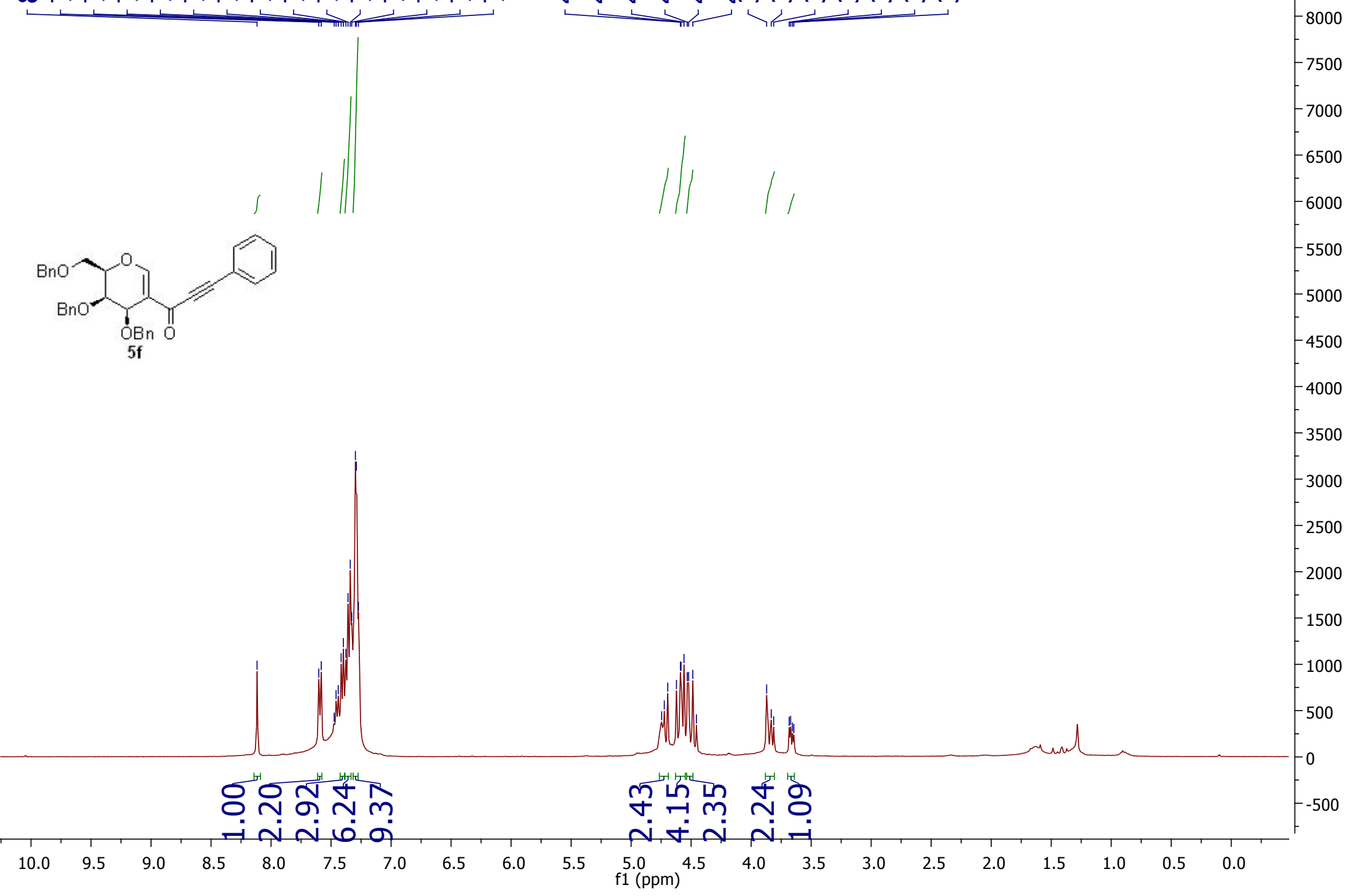




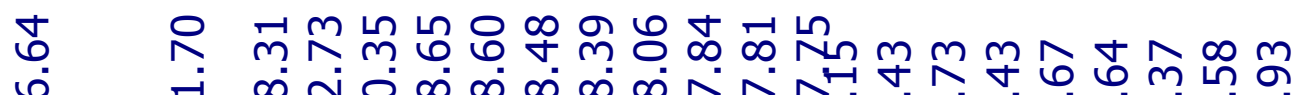

ம. $\quad$ ঢं

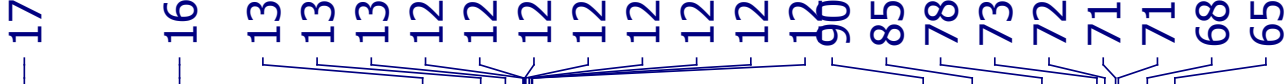
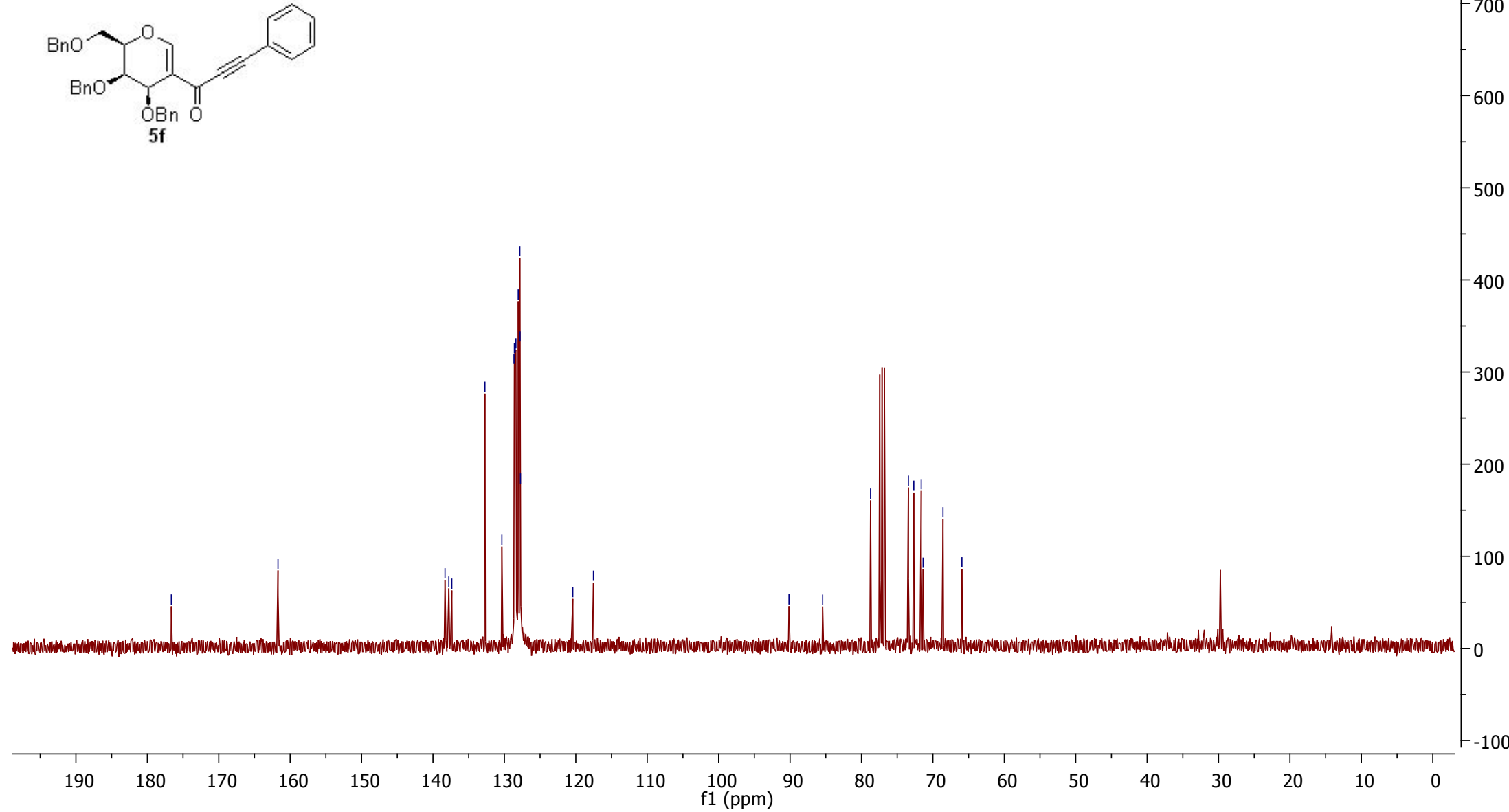


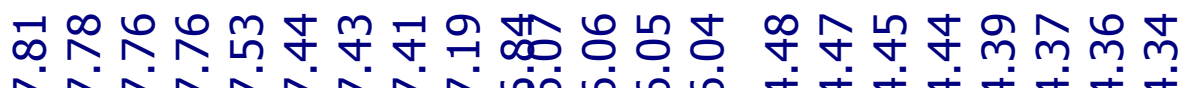
ベベベベベベベம
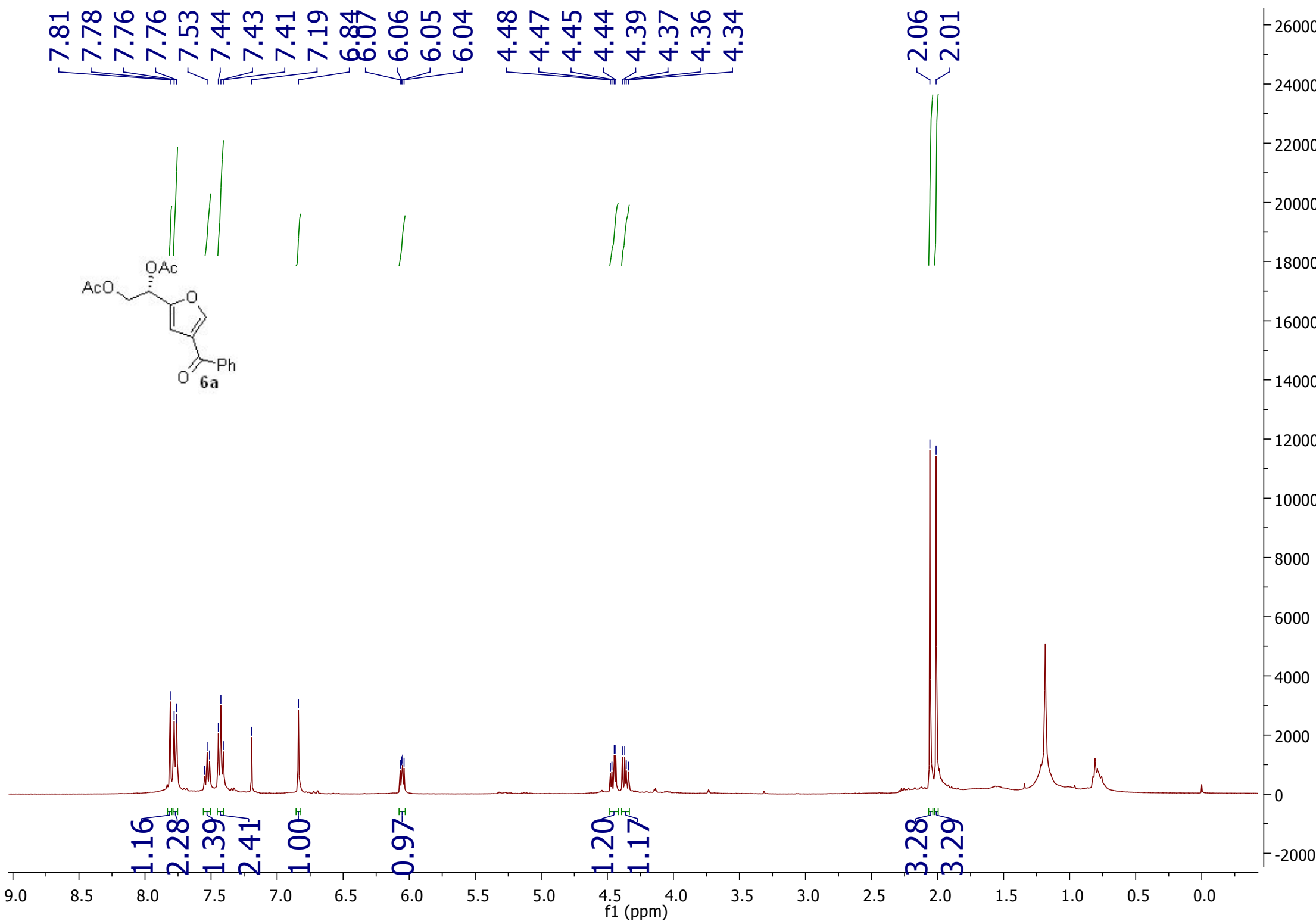


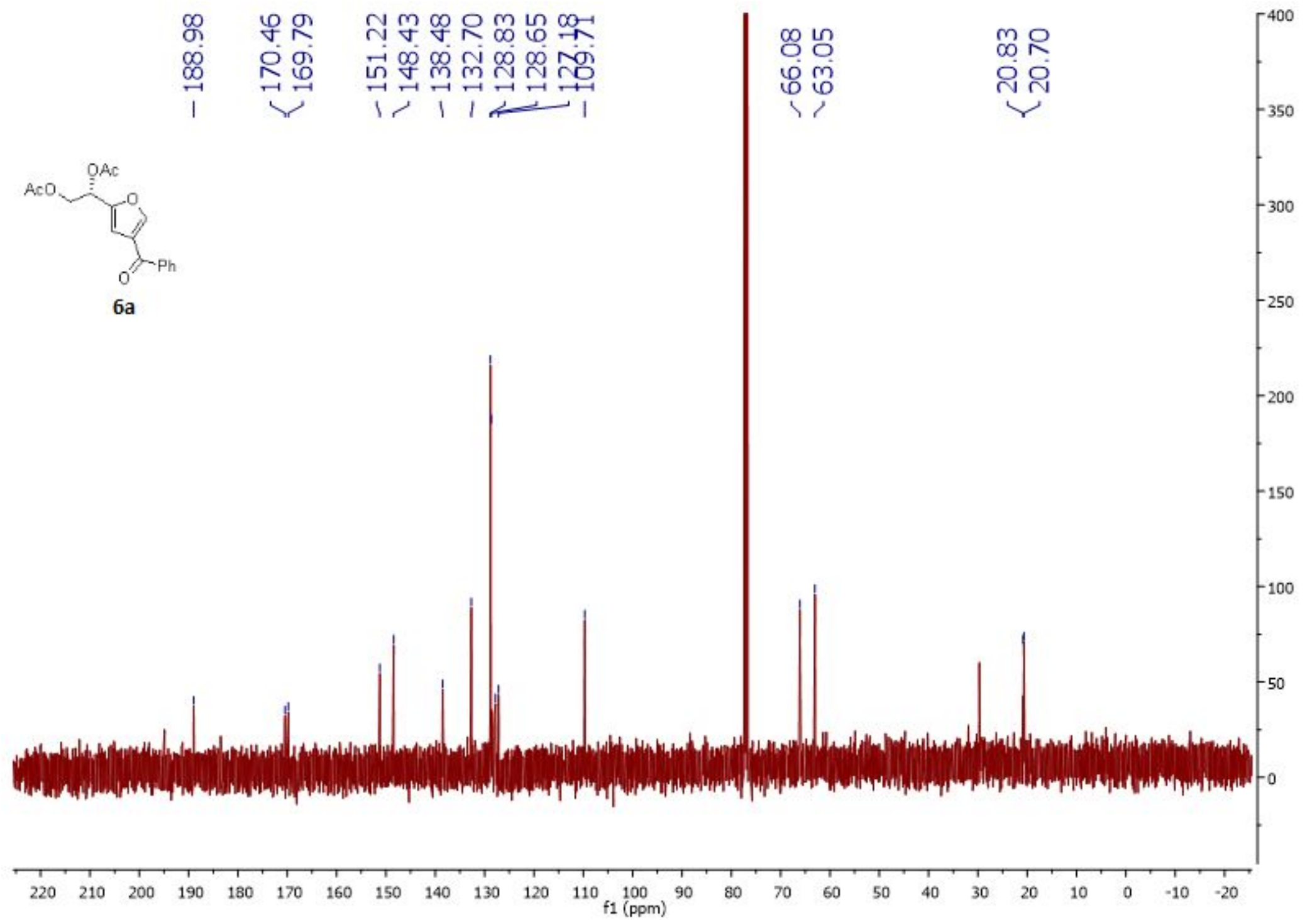


HSQC of compound 6a (Expanded version)

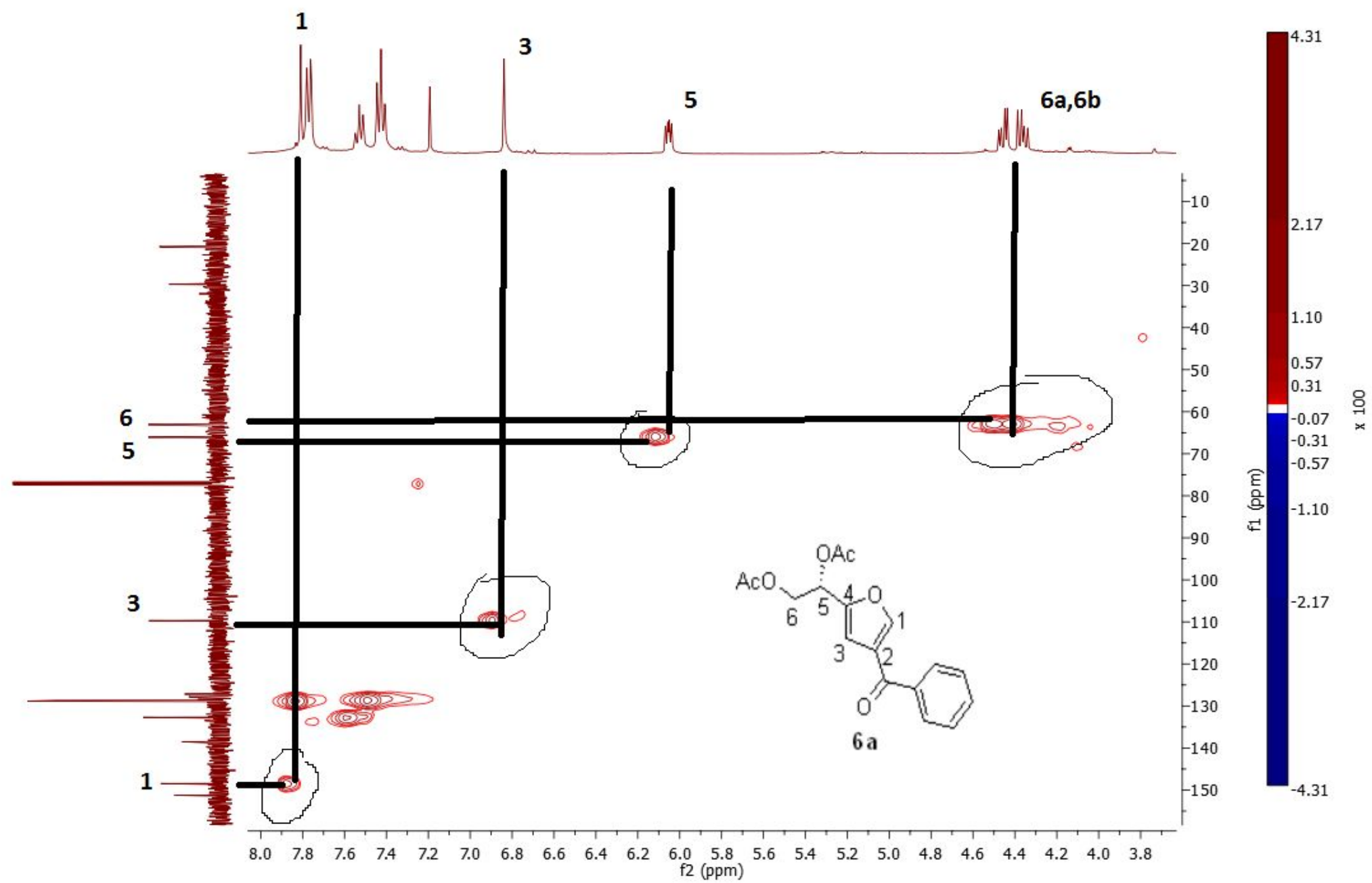




\section{HSQC of compound 6a (Normal version)}

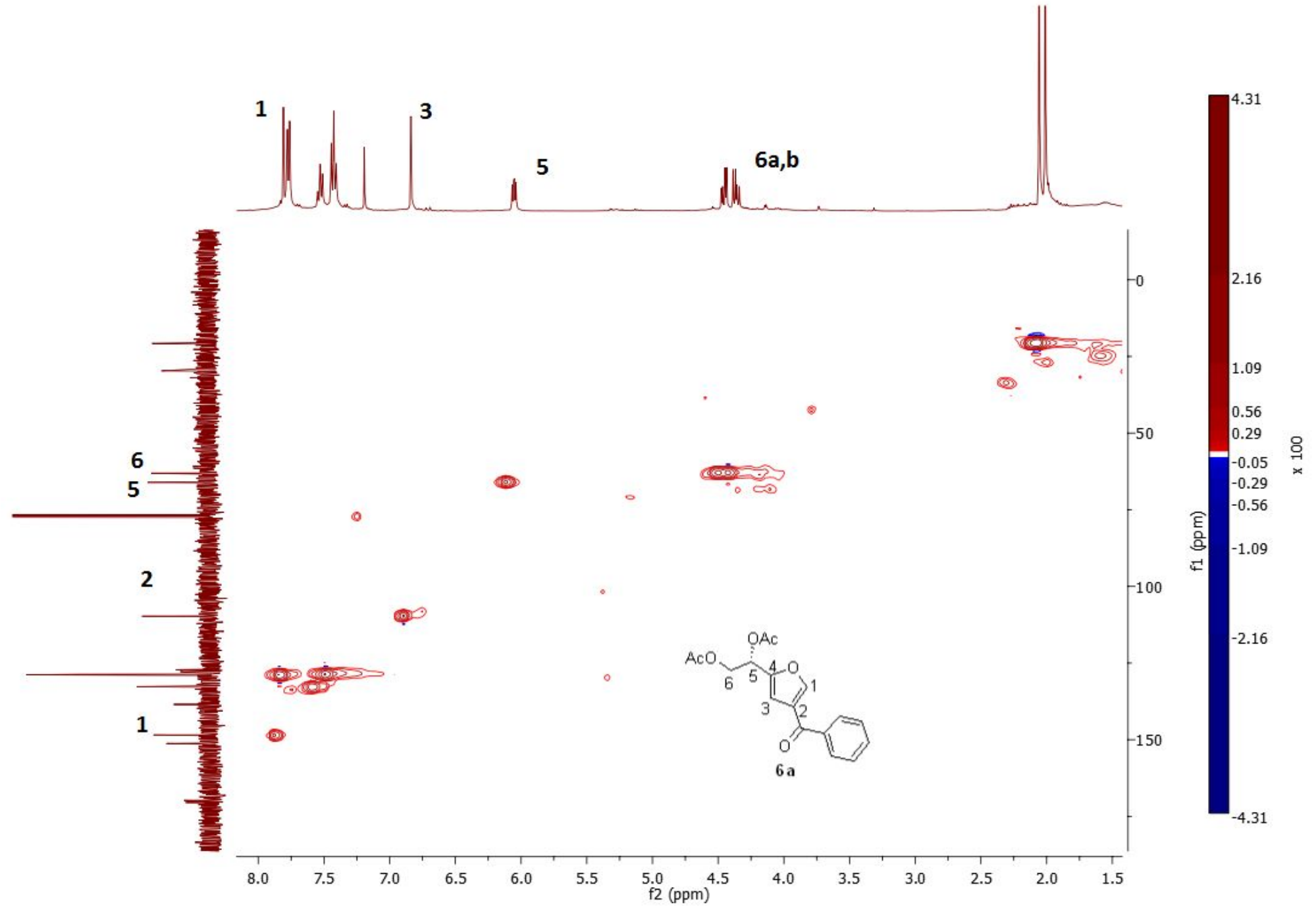




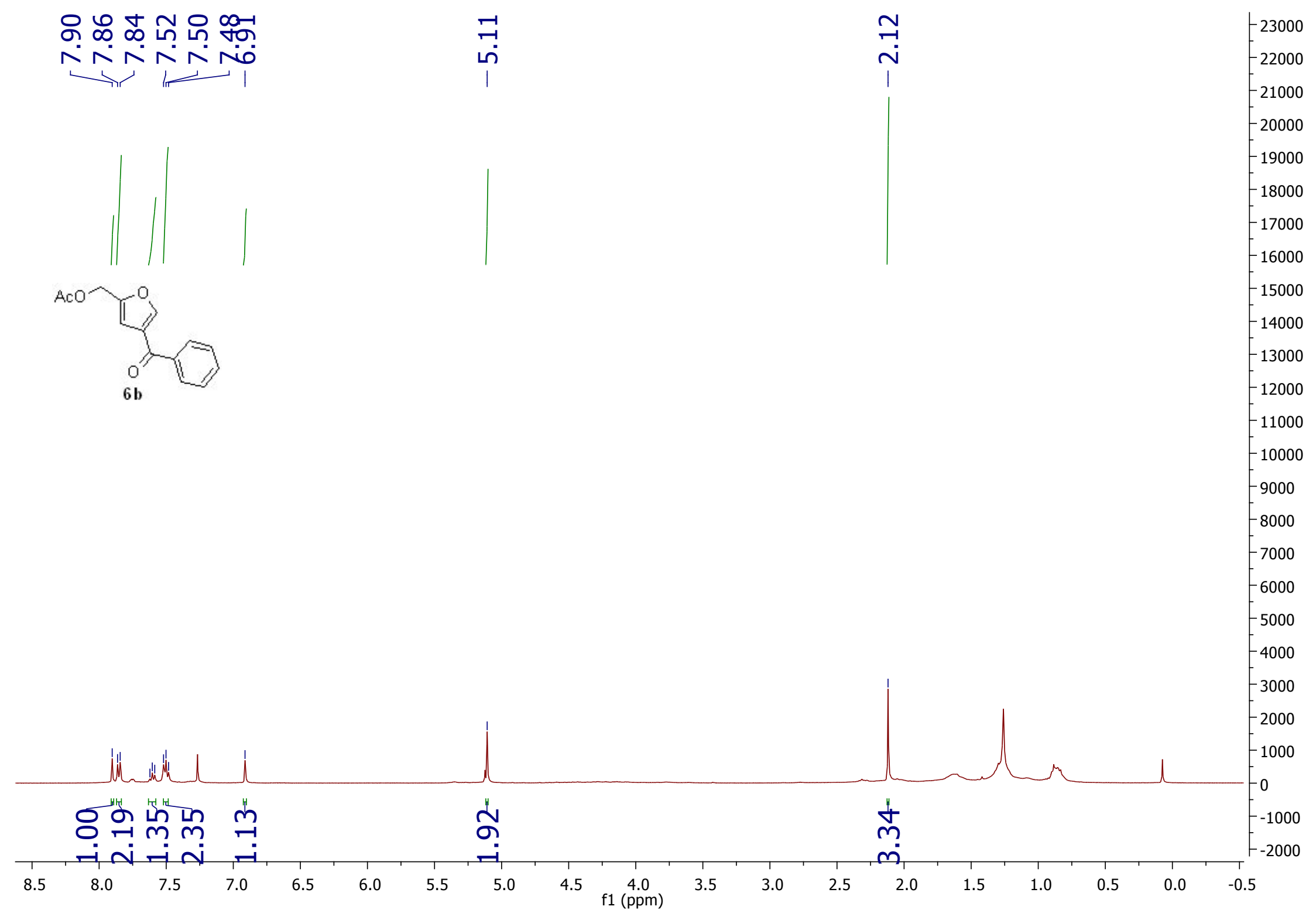




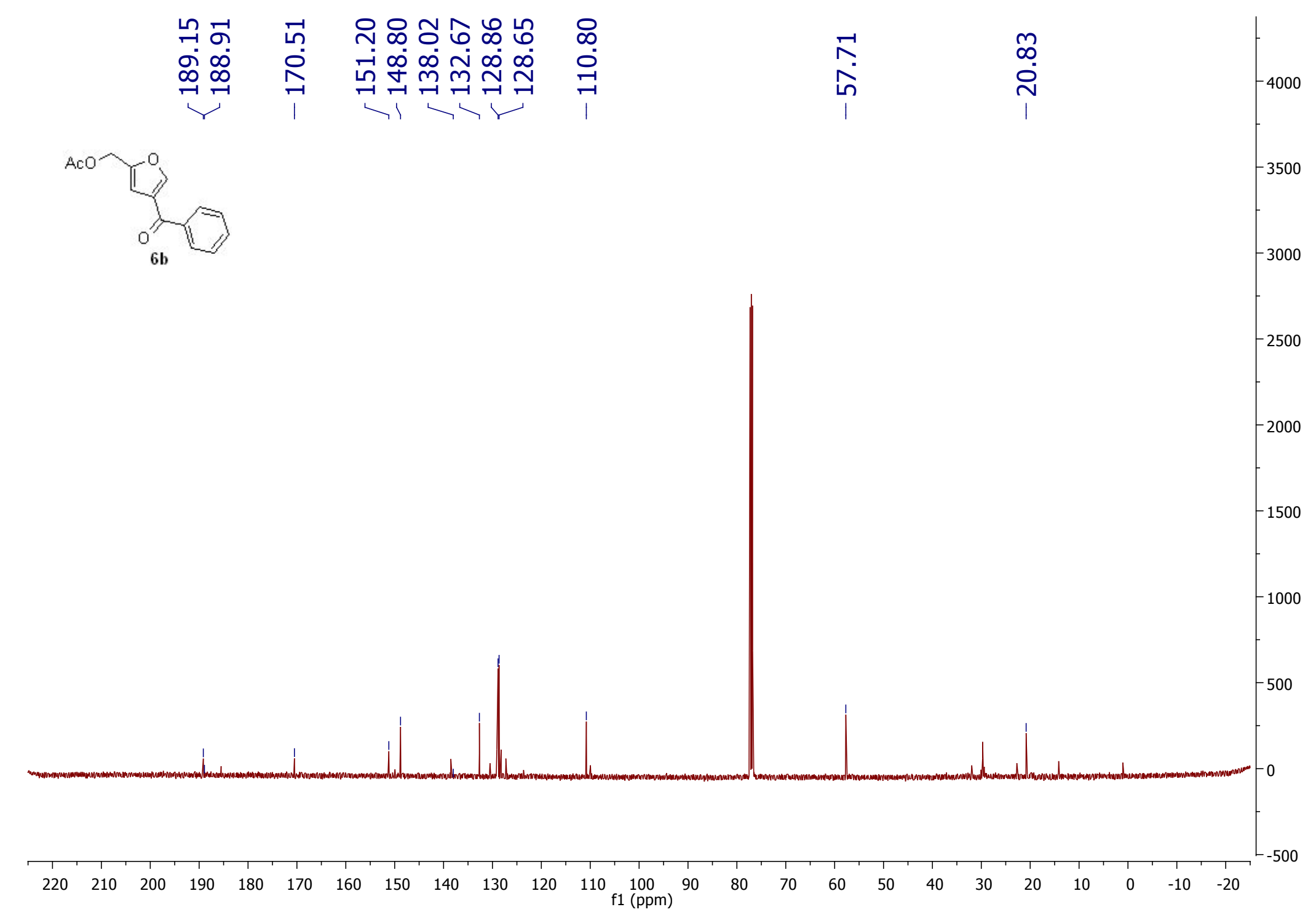




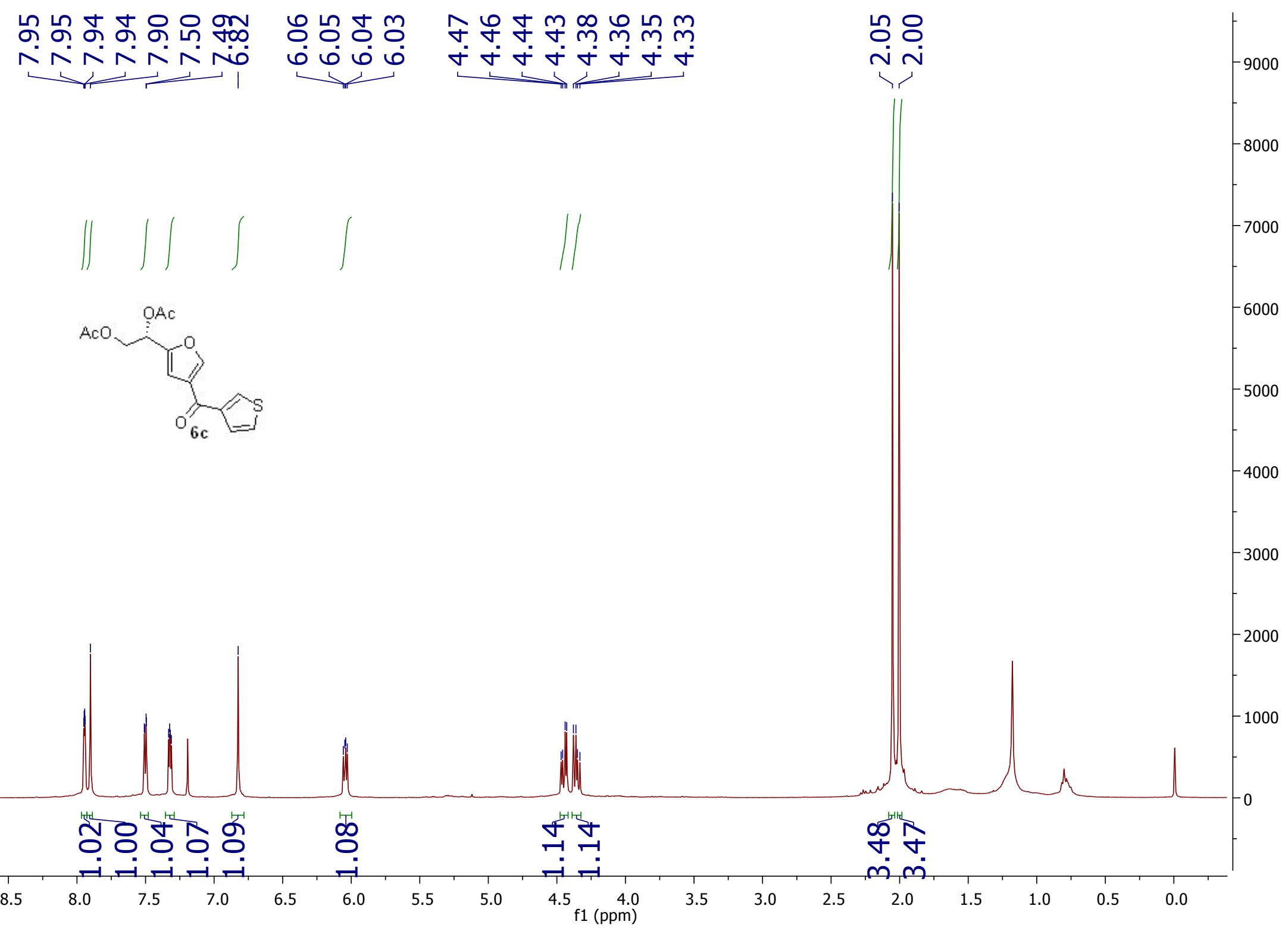




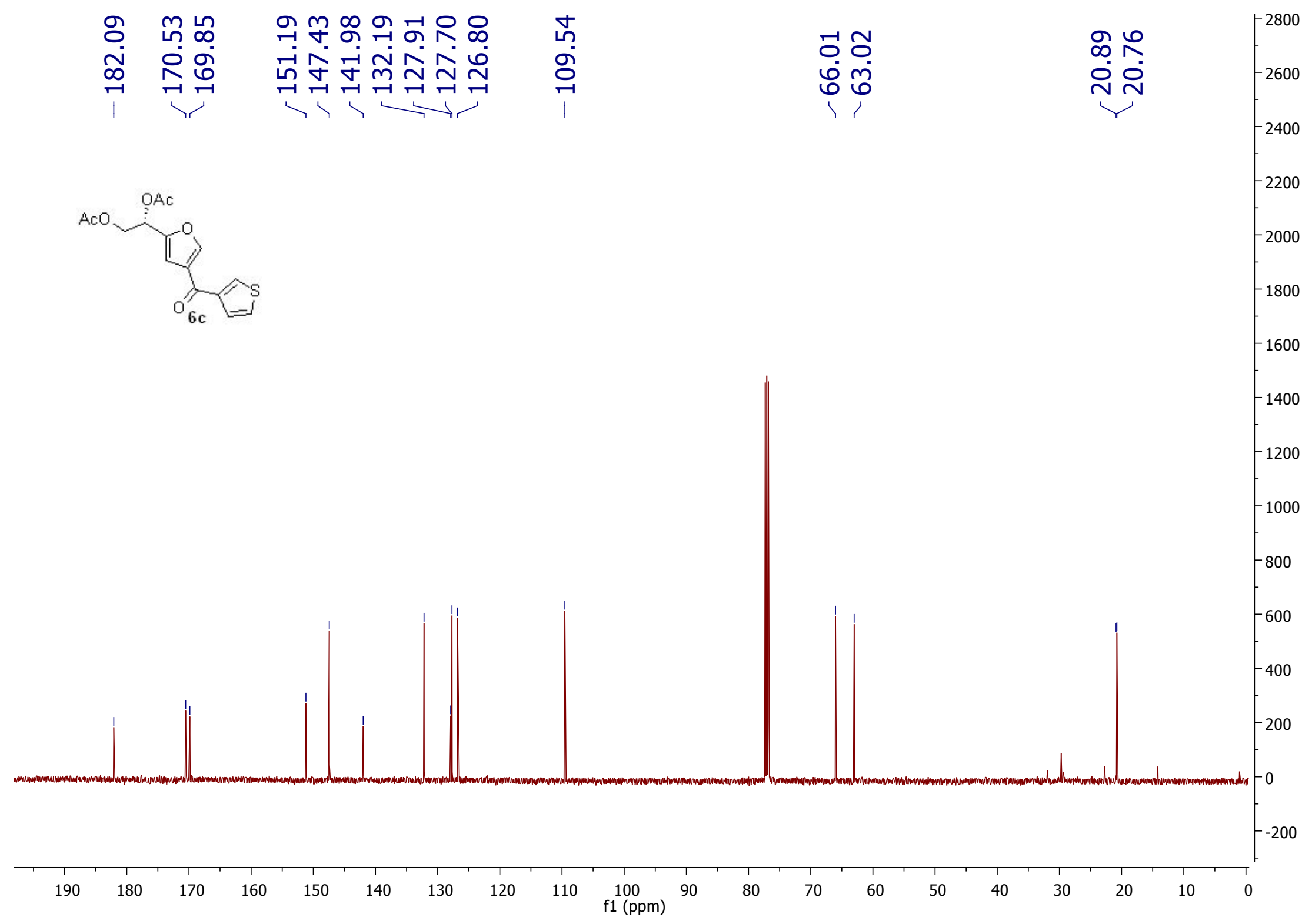




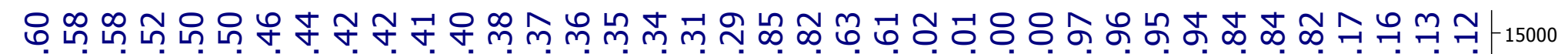

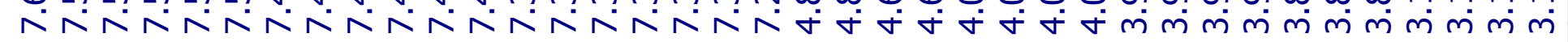






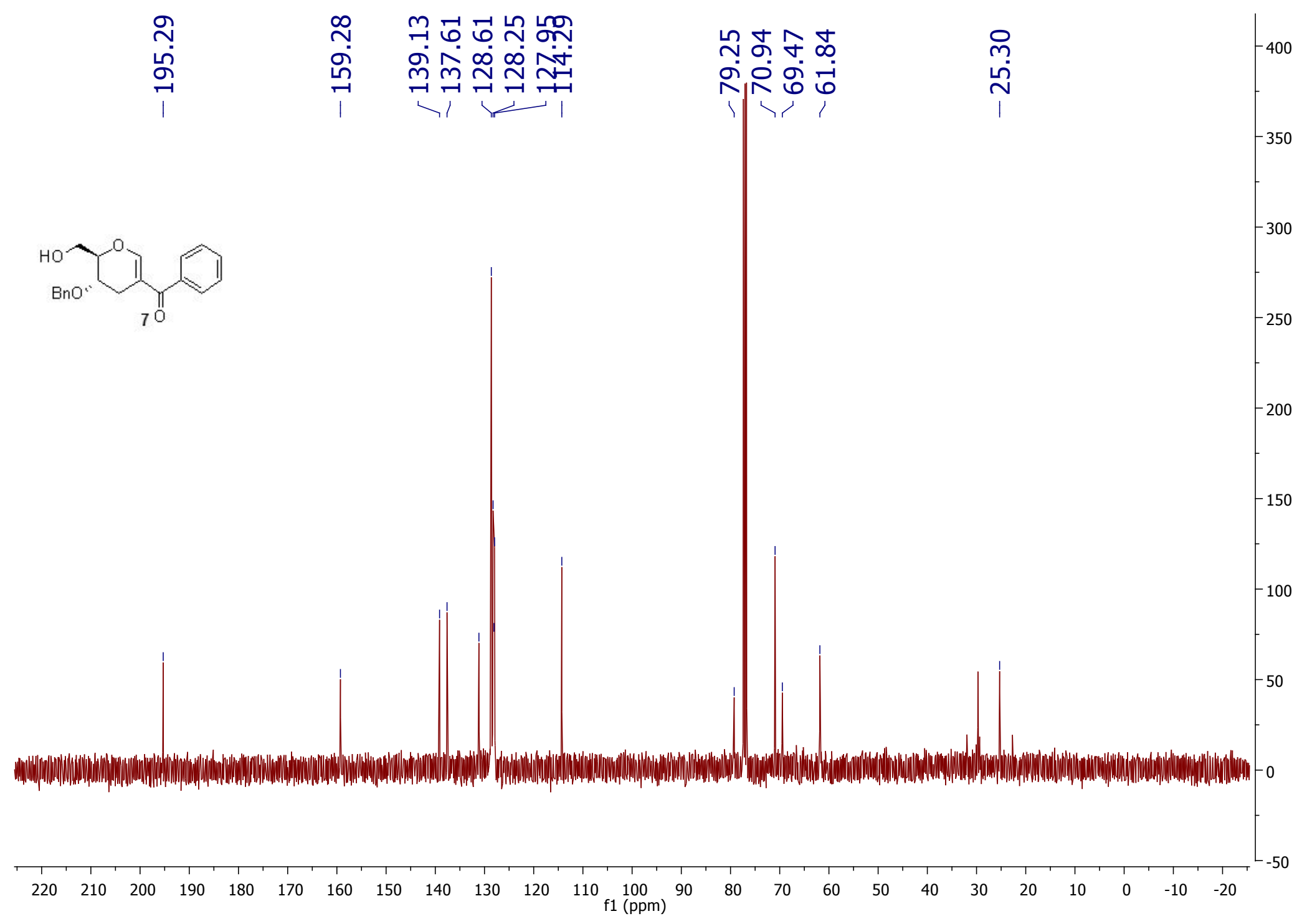




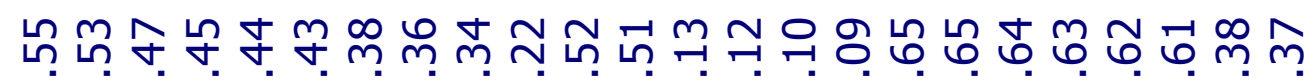

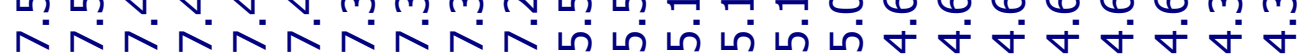

겅ㅇㅇ

$-50000$ L L

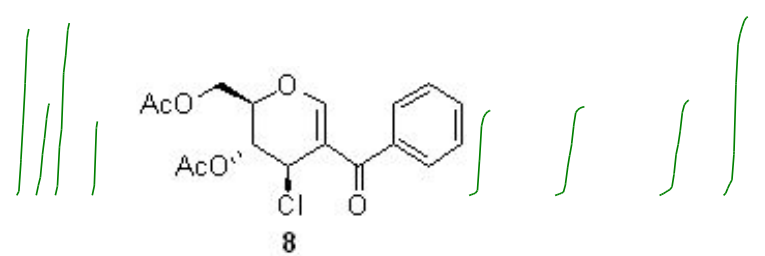

in

$-45000$

40000

40000

$-35000$

$-30000$

25000

$-20000$

$-15000$
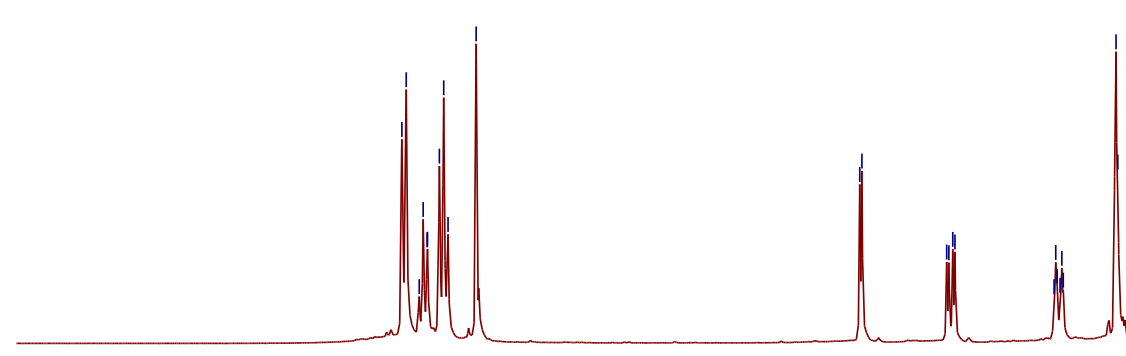

बin

80

में त्रं

, تं

$\begin{array}{lllllllll}9.0 & 8.5 & 8.0 & 7.5 & 7.0 & 6.5 & 6.0 & 5.5 & 5.0 \begin{array}{c}4.5 \\ \mathrm{f} 1(\mathrm{ppm})\end{array}\end{array}$

हit"

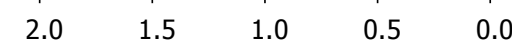




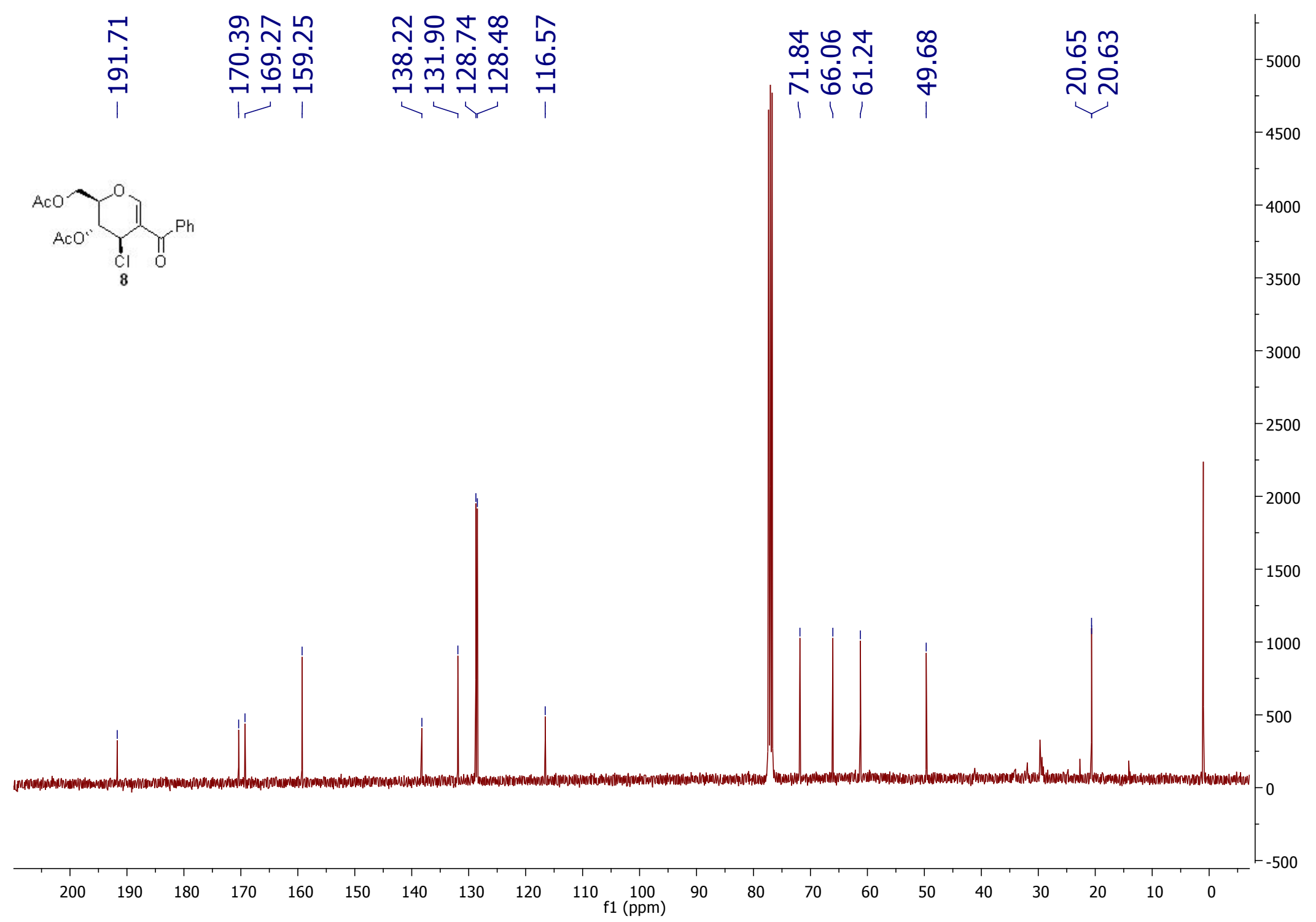




\section{HSQC of compound 8 (Normal Version)}

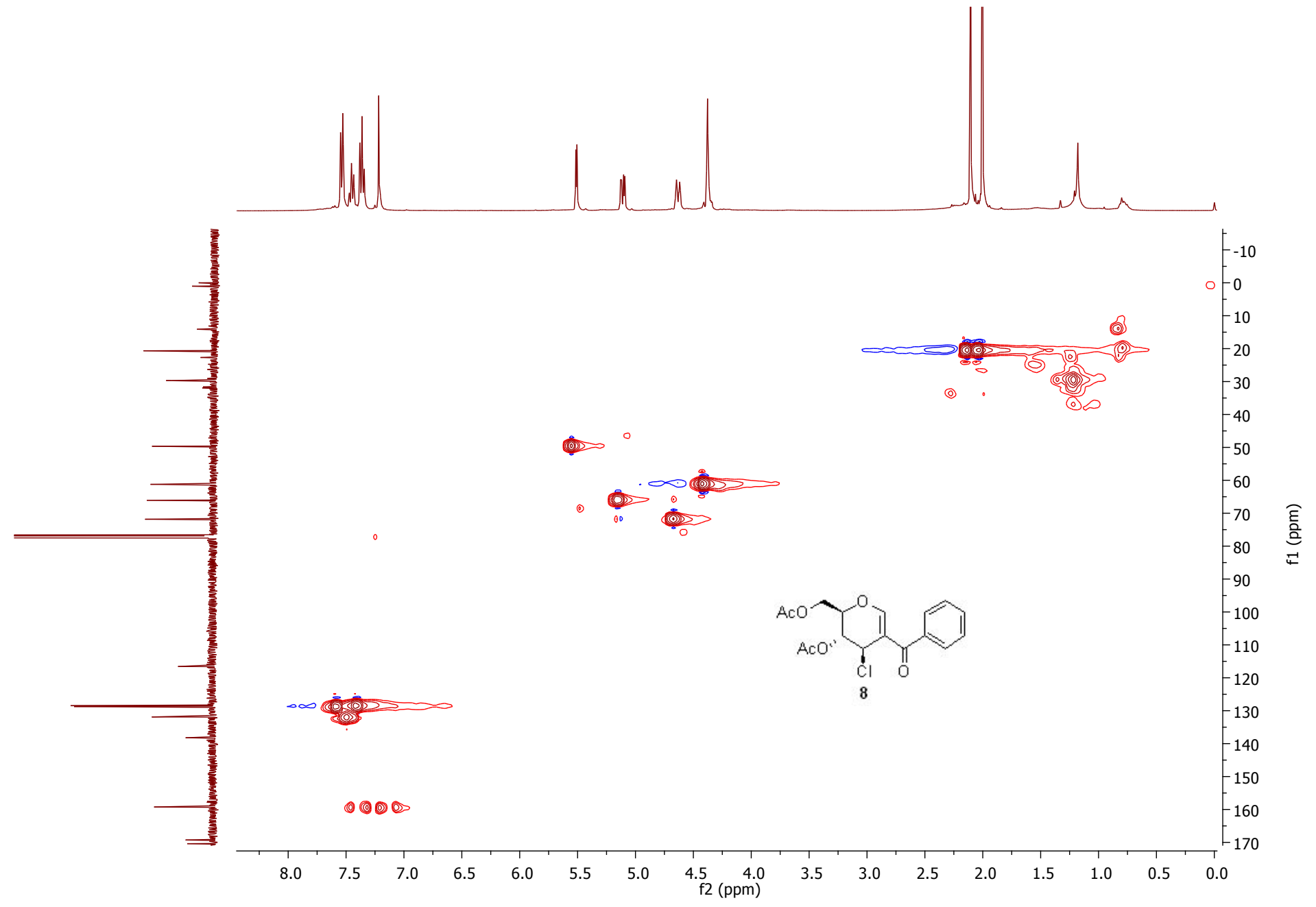


HSQC of compound 8 (Expanded Version)

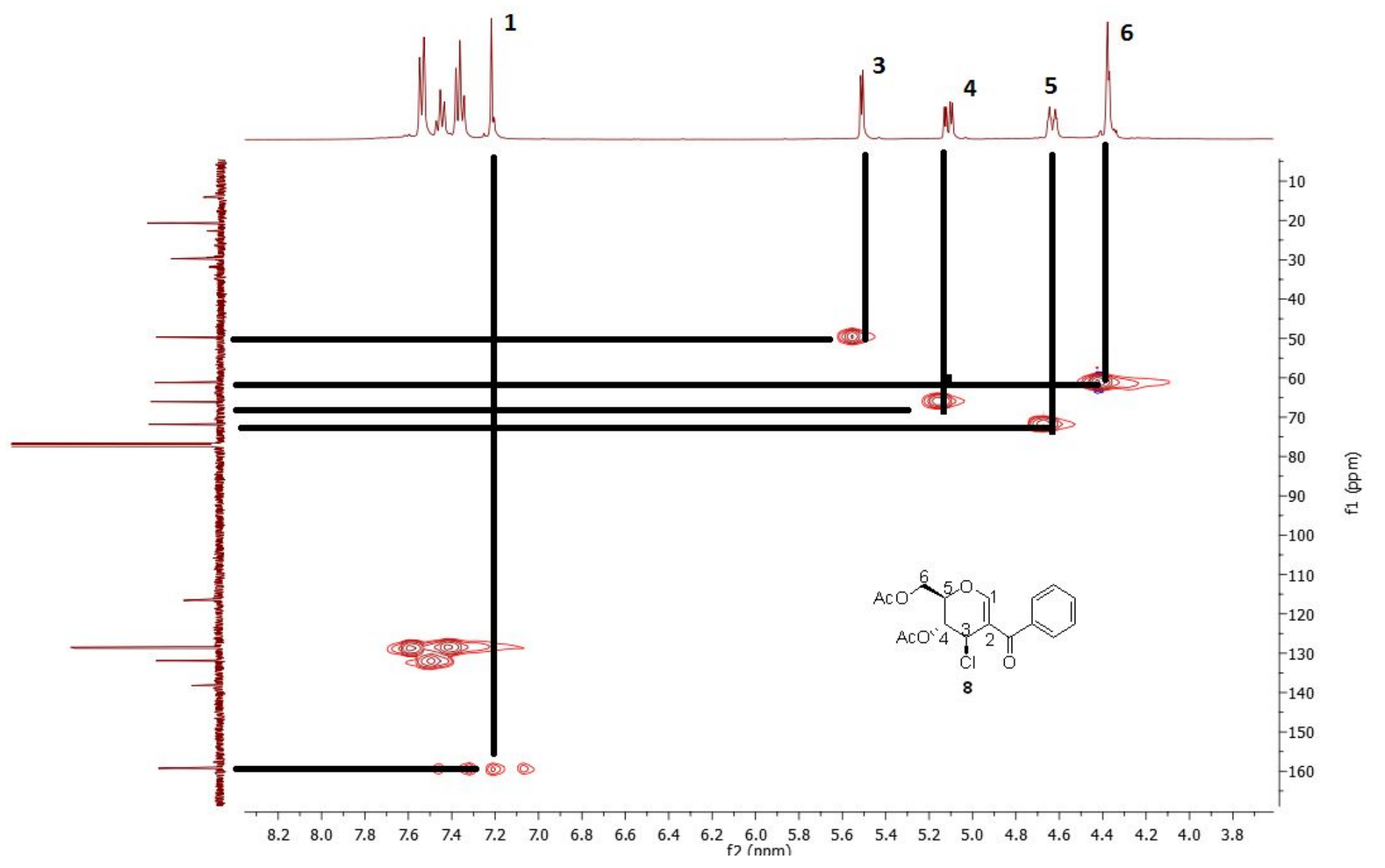


NOESY of compound 8 (Expanded Version)

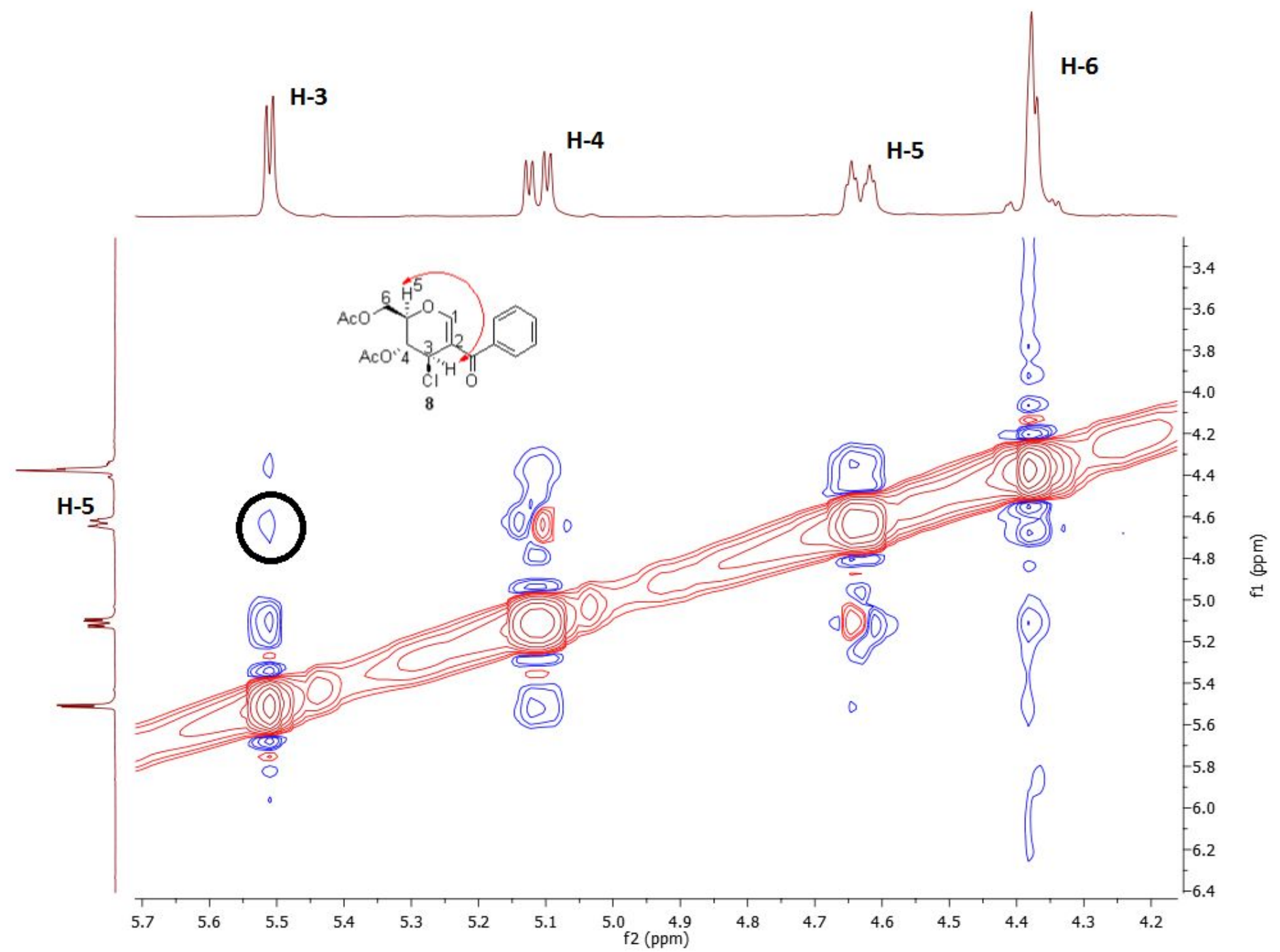


NOESY of compound 8 (Normal Version)

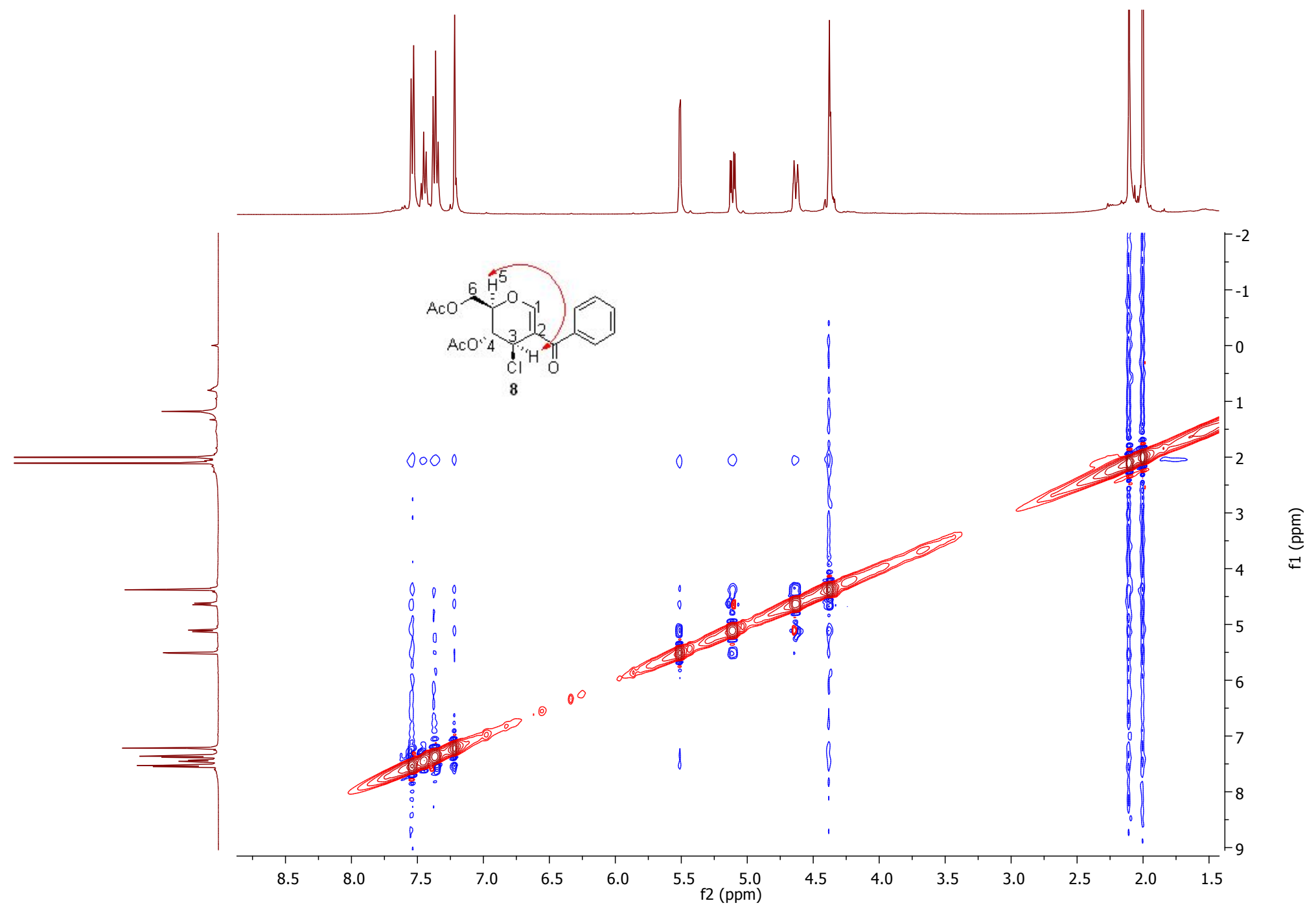




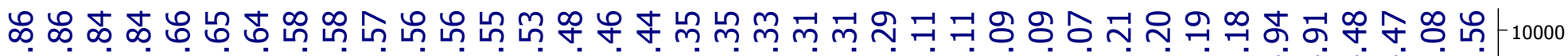

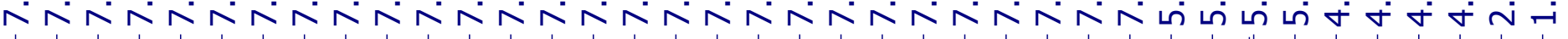
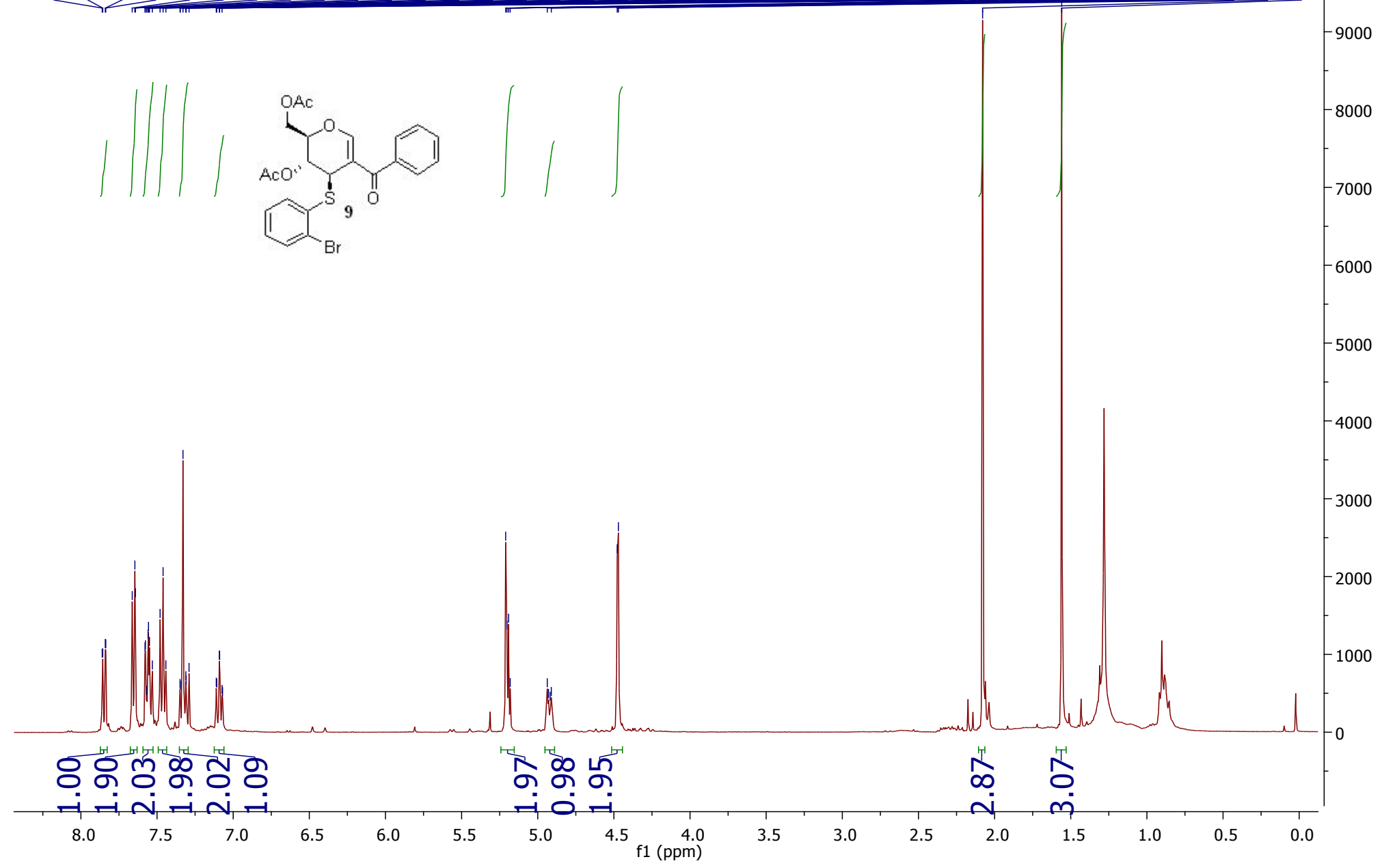


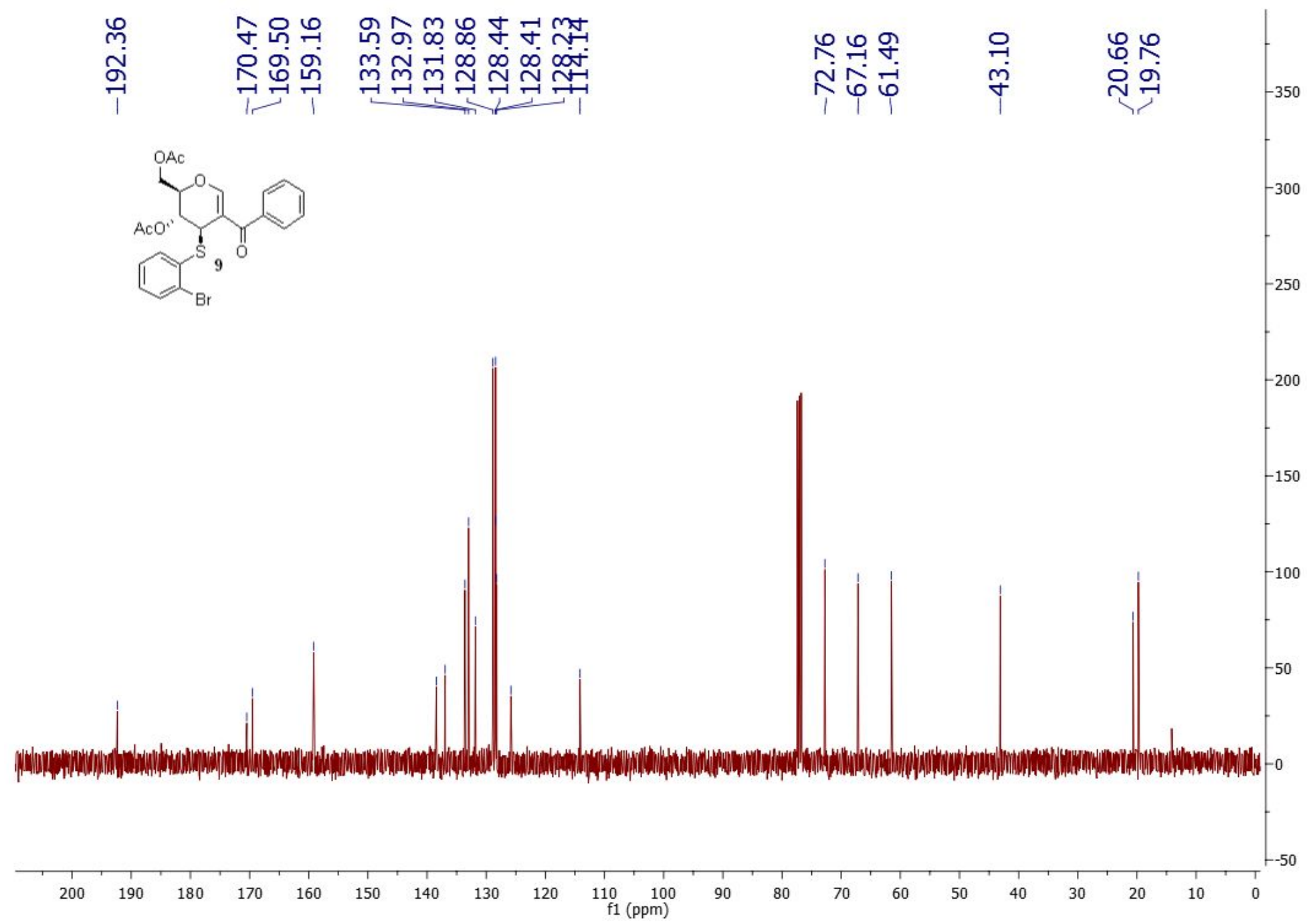




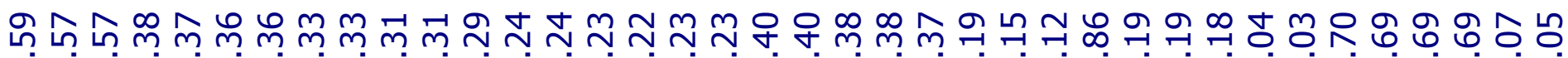

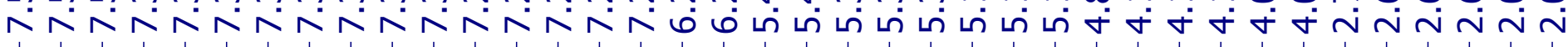
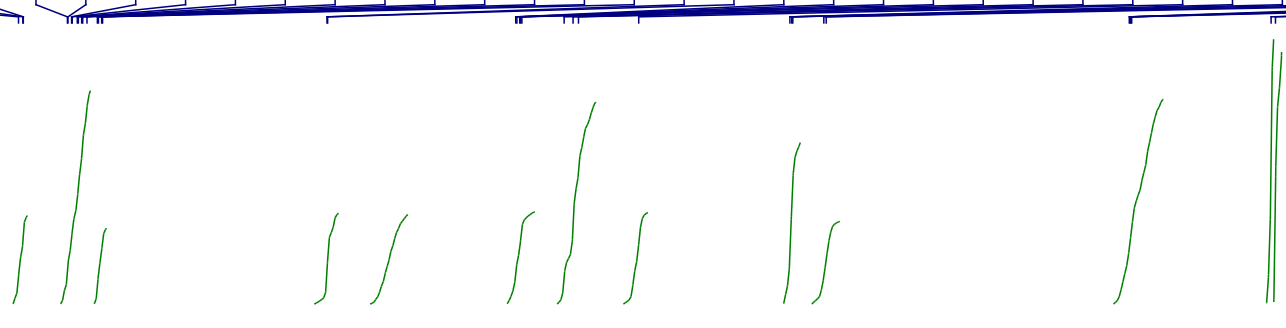

\section{0}<smiles>C=CC[C@H]1OC(COC(C)=O)C([Ge])=CC1C(=O)c1ccccc1Br</smiles>

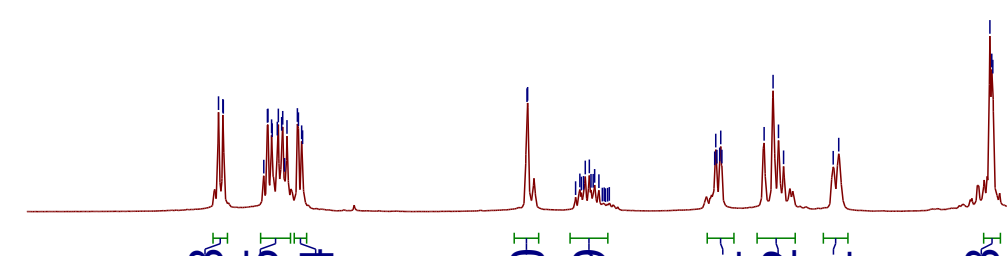

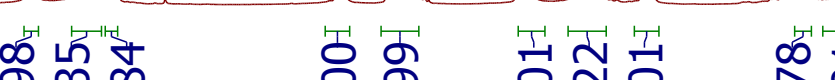
\%) $\infty$ อ. อ. ำ 





스숫ำ

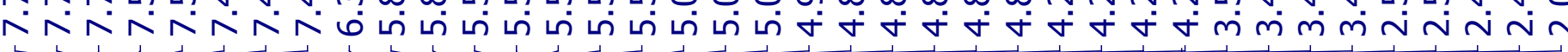
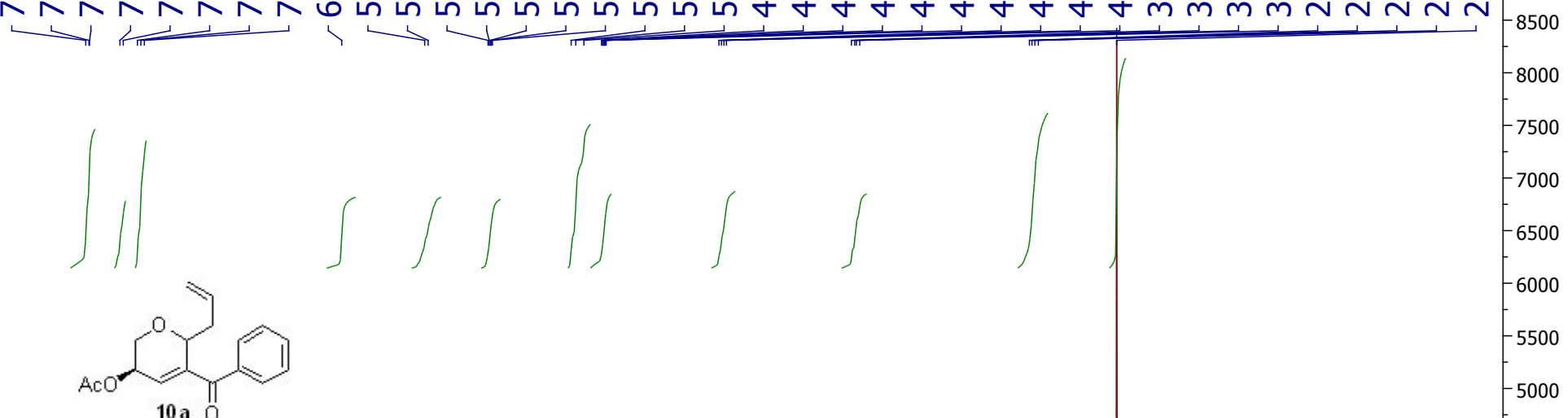

10a

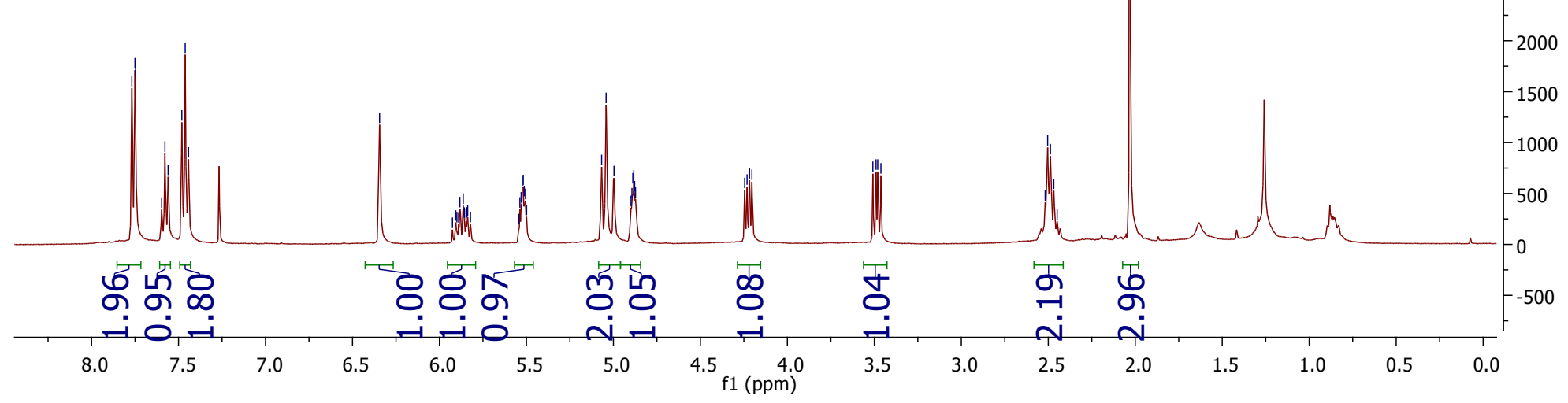




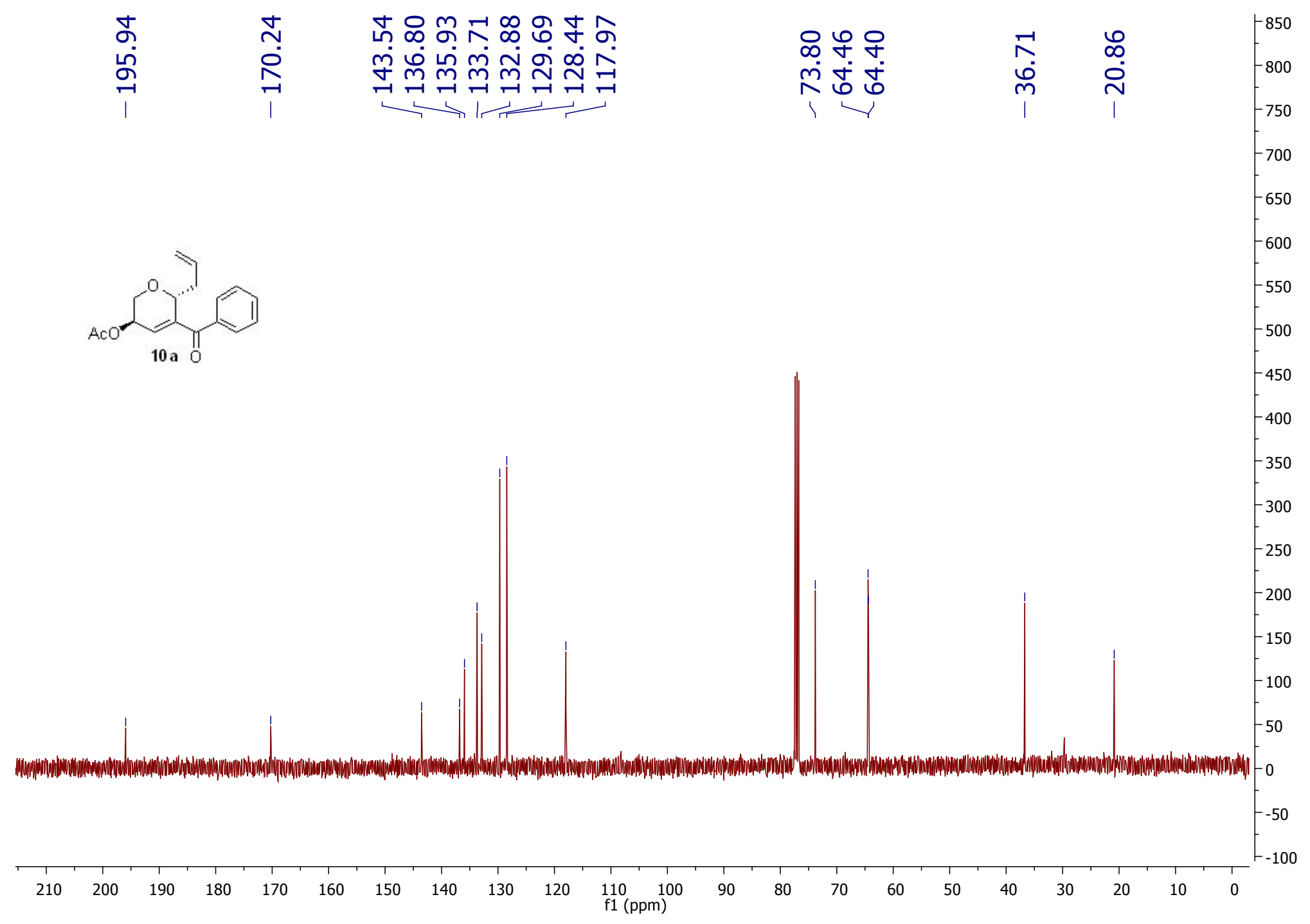




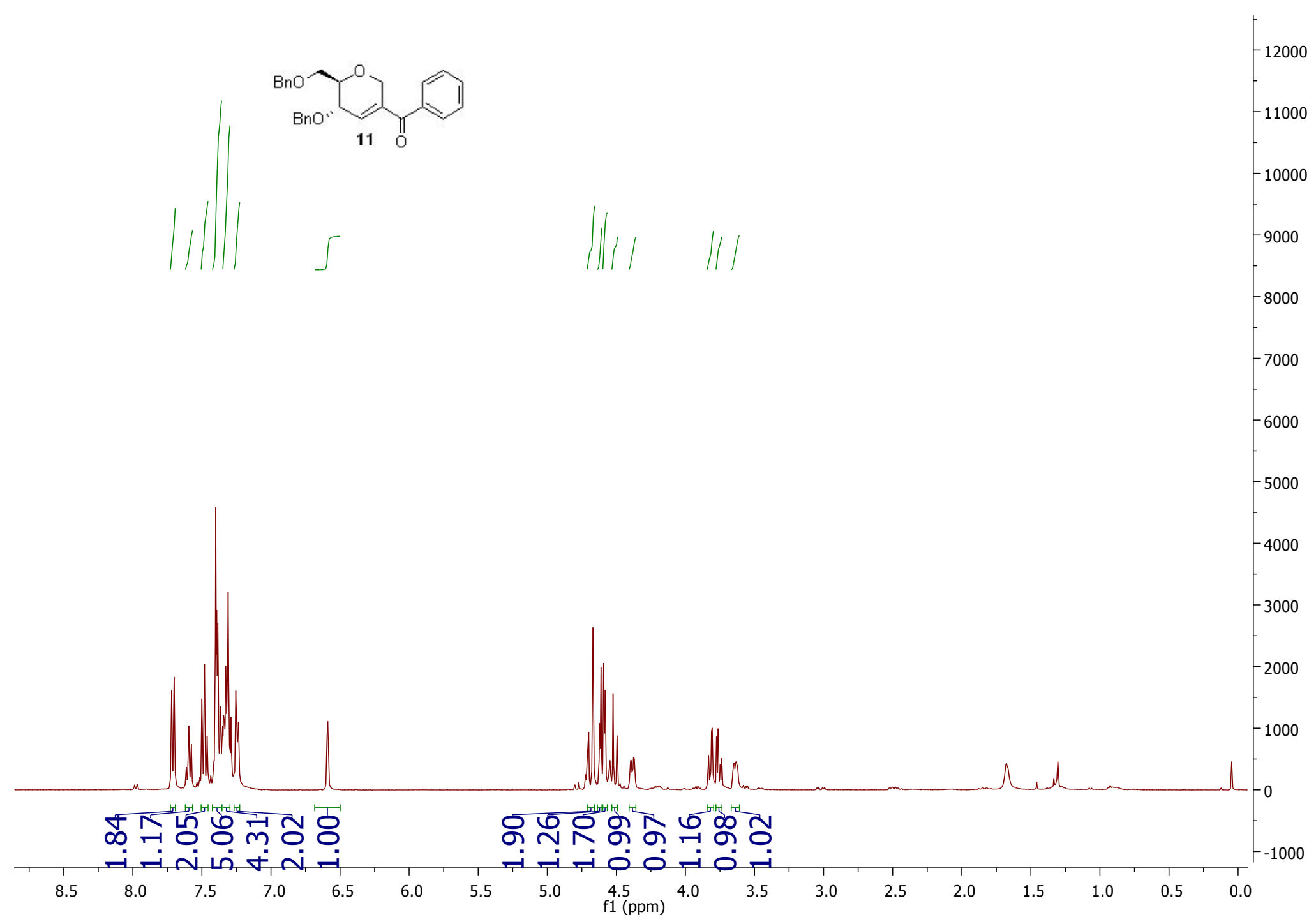




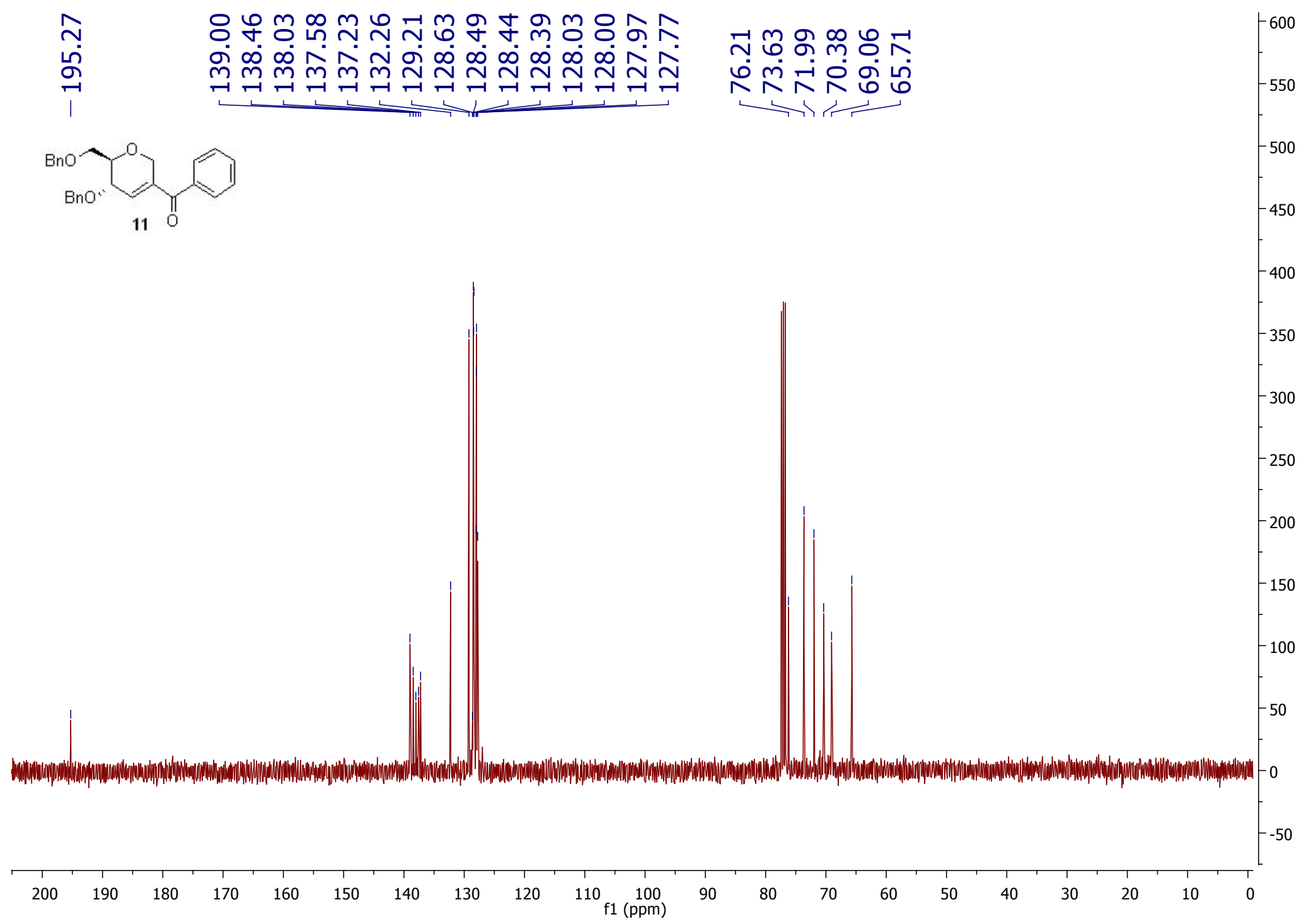




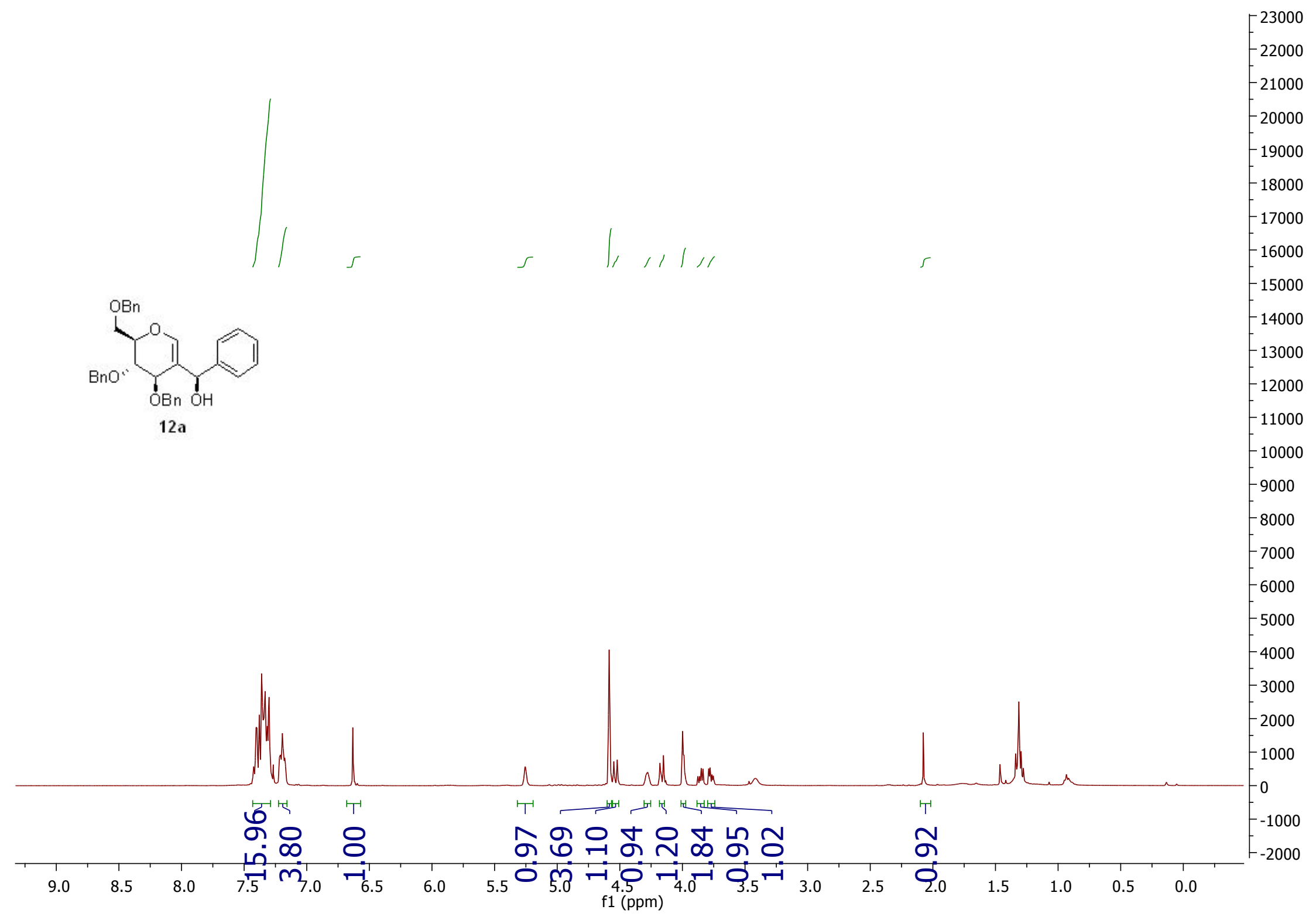




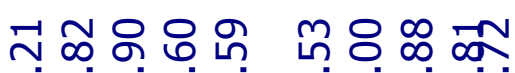

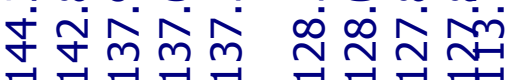

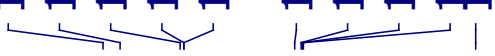

응ำㅅำ

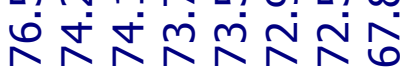

$-14000$

13000

12000

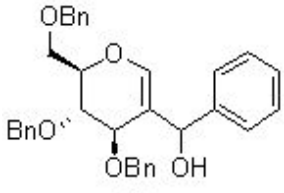

11000

10000

9000

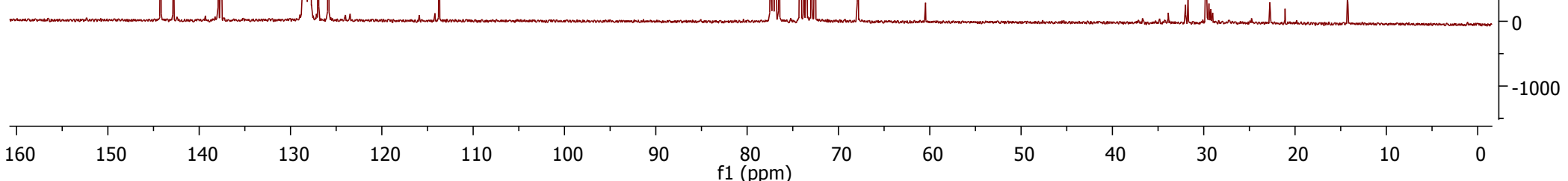


ิㅗำ

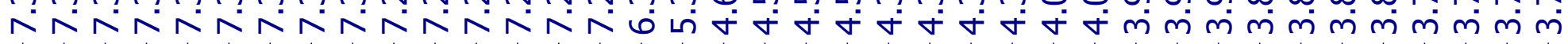

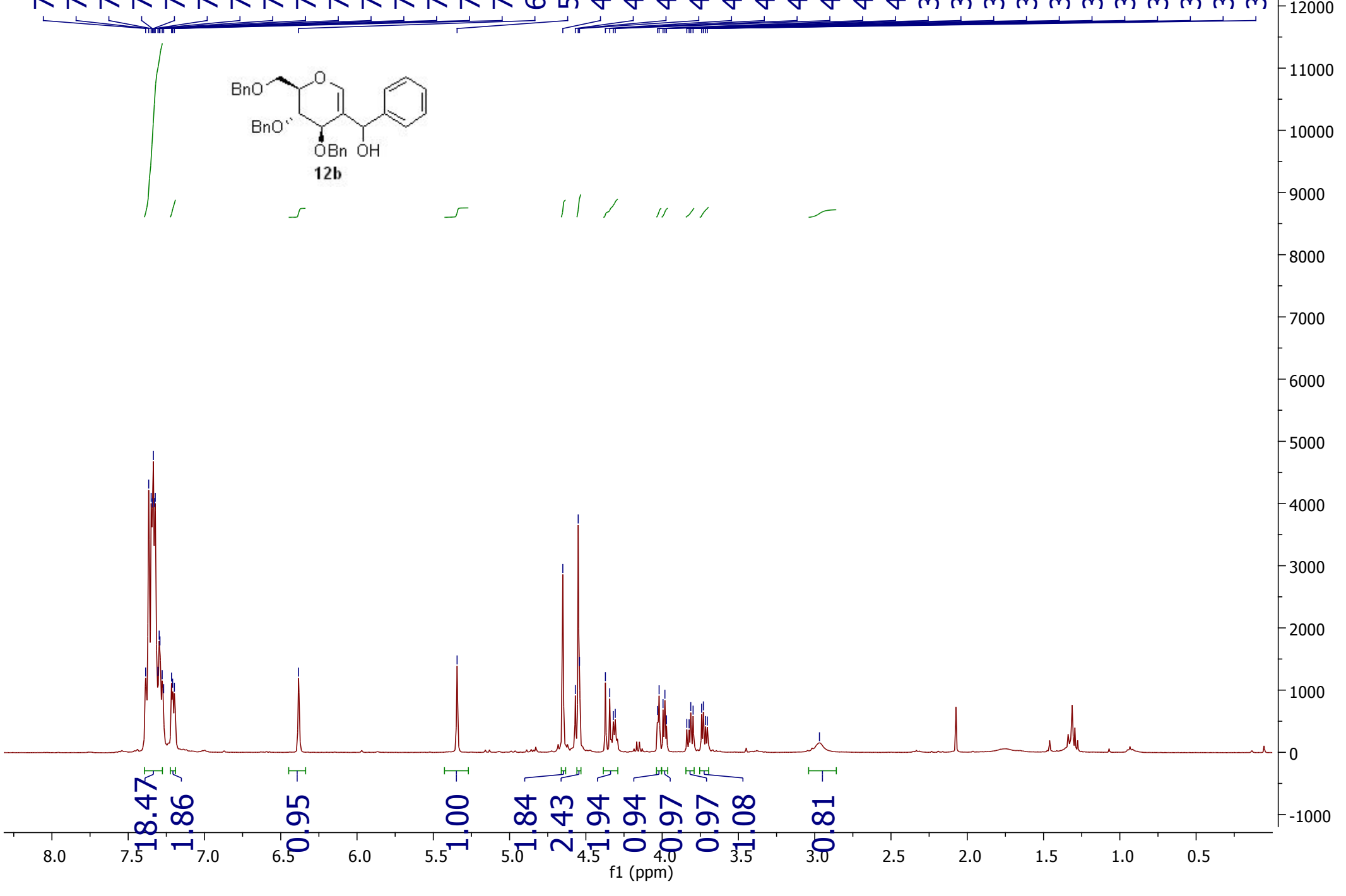




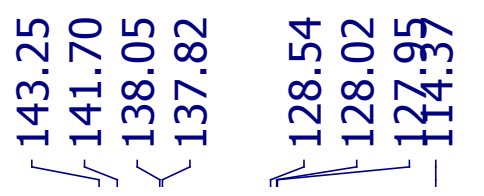

๗

ஸ்

$\wedge N \aleph N$

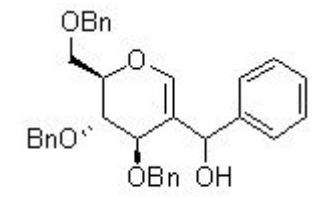

12b

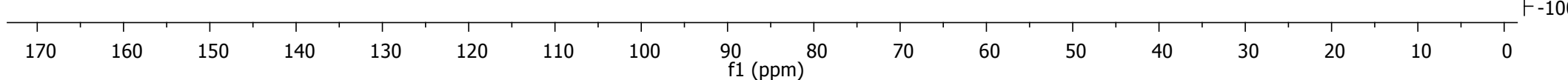


